\title{
PROPAGATION OF 1-D WAVES IN REGULAR DISCRETE HETEROGENEOUS MEDIA: A WIGNER MEASURE APPROACH
}

\author{
AURORA MARICA AND ENRIQUE ZUAZUA
}

\begin{abstract}
In this article, we describe the propagation properties of the one-dimensional wave and transport equations with variable coefficients semi-discretized in space by finite difference schemes on non-uniform meshes obtained as diffeomorphic transformations of uniform ones. In particular, we introduce and give a rigorous meaning to notions like the principal symbol of the discrete wave operator and the corresponding bi-characteristic rays. The main mathematical tool we employ is the discrete Wigner transform, which, in the limit as the mesh size parameter tends to zero, yields the so-called Wigner (semi-classical) measure. This measure provides the dynamics of the bi-characteristic rays, i.e., the solutions of the Hamiltonian system describing the propagation, in both physical and Fourier spaces, of the energy of the solution to the wave equation. We show that, due to dispersion phenomena, the high-frequency numerical dynamics does not coincide with the continuous one. Our analysis holds for $C^{0,1}(\mathbb{R})$-coefficients and non-uniform grids obtained by means of $C^{1,1}(\mathbb{R})$-diffeomorphic transformations of a uniform one. We also present several numerical simulations that confirm the predicted paths of the space-time projections of the bi-characteristic rays. Based on the theoretical analysis and simulations, we describe some of the pathological phenomena that these rays might exhibit as, for example, their reflection before touching the boundary of the space domain. This leads, in particular, to the failure of the classical properties of boundary observability of continuous waves, arising in control and inverse problems theory.
\end{abstract}

\section{INTRODUCTION AND MAIN RESULTS}

1.1. Motivation. This paper is devoted to analyze the propagation properties of discrete waves solving the finite difference numerical approximations for two basic $1-d$ wave propagation models: the first-order transport equation and the second-order wave equation. This is done on non-uniform meshes. The main contribution of the paper is to introduce a suitable notion of symbol allowing to construct bi-characteristic and characteristic rays propagating the energy of solutions.

The work developed in this article is motivated by control and inverse problems. Indeed, the well-known boundary controllability and identifiability properties of solutions of the wave equation hold because of the fact that the energy of solutions is driven by characteristics that reach the boundary where the controllers or observers are placed. This property is well-known to generally fail for numerical schemes because of the pathological behavior of high-frequency numerical spurious solutions (see [11, [12, [35]). But this analysis has been developed, so far, only in the context of numerical approximations on uniform meshes. The goal of this paper is to develop the fundamental notions and tools of microlocal analysis allowing to handle numerical discretization schemes on non-uniform meshes. As we shall see, the non-uniformity of the mesh adds further dispersion properties to the numerical solutions.

1.1.1. The continuous transport equation. The first basic model analyzed in this paper is the scalar transport equation

$$
\varrho(y) \partial_{t} u(y, t)+\partial_{y} u(y, t)=0, y \in \mathbb{R}, t>0, \quad u(y, 0)=u^{0}(y) .
$$

September 4, 2013. All authors were partially supported by the ERC Advanced Grant FP7-246775 NUMERIWAVES, Grant MTM2011-29306 of MICINN Spain, Project PI2010-04 of the Basque Government, and the ESF Research Networking Programme OPTPDE. Additionally, the work of the first author was supported by two grants of the Ministry of National Education (CNCS-UEFISCDI), projects number PN-II-ID-PCE-2012-4-0021 "Variable Exponent Analysis: Partial Differential Equations and Calculus of Variations" and PN-II-ID-PCE-2011-3-0075 "Analysis, control and numerical approximations of PDEs" and MOBIS - Mathematical Optimization and Applications in Biomedical Sciences of the FWF - Austrian Science Fund. This work was mainly realized during the research stay of the first author in BCAM as postdoctoral fellow within the NUMERIWAVES Project between November 2010 - August 2012. 
Here and in what follows, $\partial_{t}$ and $\partial_{y}$ are the first-order time and space derivatives and $\varrho(y) \geq \varrho^{-}>0$, for all $y \in \mathbb{R}$, is an $L^{\infty}(\mathbb{R})$-function. Under these assumptions, the following energy (coinciding with the weighted $L^{2}(\mathbb{R} ; \varrho)$-norm of the solution $\left.u(y, t)\right)$ is conserved in time:

$$
\mathcal{E}_{\varrho}(u(\cdot, t))=\int_{\mathbb{R}}|u(y, t)|^{2} \varrho(y) d y:=\mathcal{E}_{\varrho}\left(u^{0}\right) .
$$

For all strictly positive density functions $\varrho \in C^{0,1}(\mathbb{R})$, the transport equation 1.1 can be uniquely solved by the method of characteristics as $u(y(t), t)=u^{0}\left(y_{0}\right)$, where $y(t)$ are the so-called characteristic curves solving the first-order ordinary differential equation (ODE)

$$
y^{\prime}(t)=c(y(t)), t>0, \quad y(0)=y_{0} \in \mathbb{R}, \text { with } c(y):=1 / \varrho(y) .
$$

By ${ }^{\prime}$ we denote the derivative of a function depending on only one variable. When $\varrho \equiv 1$, the solutions of (1.3) are straight lines of the form $y(t)=y_{0}+t$, for all $y_{0} \in \mathbb{R}$ and $t \geq 0$. Accordingly, the solutions of the transport equation 1.1 take the form $u(y, t)=u^{0}(y-t)$.

1.1.2. The continuous wave equation. The second main model analyzed in this paper is the one-dimensional wave equation

$$
\rho(y) \partial_{t}^{2} u(y, t)-\partial_{y}\left(\sigma(y) \partial_{y} u\right)(y, t)=0, y \in \mathbb{R}, \quad u(y, 0)=u^{0}(y), \partial_{t} u(y, 0)=u^{1}(y), y \in \mathbb{R},
$$

where $\partial_{t}^{2}$ is the second-order time derivative operator and $\rho, \sigma>0$ are $L^{\infty}(\mathbb{R})$-functions. The total energy below is conserved in time:

$$
\mathcal{E}_{\rho, \sigma}\left(u^{0}, u^{1}\right):=\frac{1}{2} \int_{\mathbb{R}}\left(\rho(y)\left|\partial_{t} u(y, t)\right|^{2}+\sigma(y)\left|\partial_{y} u(y, t)\right|^{2}\right) d y .
$$

When the coefficients of the wave equation (1.4) are constant ( $\rho=\sigma \equiv 1$, for simplicity), the corresponding solution is given by the d'Alembert formula below, stating that the solution of the wave equation can be uniquely decomposed into two components, each one propagating along one of the characteristics $y \pm t$ :

$$
u(y, t)=\frac{1}{2}\left(u^{0}(y+t)+u^{0}(y-t)\right)+\frac{1}{2} \int_{y-t}^{y+t} u^{1}(z) d z .
$$

Of course, for the variable coefficients case, there is no explicit formula for solutions. However, it is well-known (cf. [1]) that the energy of initial data presenting high-frequency oscillation and/or concentration effects propagates along the characteristic rays. The role $\mathrm{f}$ these rays can be illustrated by considering highly concentrated and oscillatory initial data in 1.1) or (1.4) leading to Gaussian wave packet-type solutions concentrated, precisely, along one of the bi-characteristic lines and for which the energy localized outside any neighborhood vanishes as the wavelength parameter tends to zero ([21], [22]).

1.1.3. Wave propagation in the continuous setting and its applications. As mentioned above, an important application of the propagation of waves along characteristics comes from control theory. One of the most typical problems in control is that of exact controllability. It consists in driving the solution of the PDE under consideration (or of its numerical approximation schemes) to the equilibrium by means of an applied force, the control, localized on some subset of the domain where waves propagate as, for instance, a part or a neighborhood of the boundary or the complementary set of a bounded domain. This control problem is equivalent to the observability one, consisting in the possibility to obtain estimates of the total energy of the solutions of the uncontrolled system (1.4) in terms of the energy concentrated on the support of the control along time. For the linear wave equation with smooth coefficients, the observability problem has a positive answer if and only if the Geometric Control Condition (GCC) holds. This GCC requires all rays of Geometric Optics to enter the control/observability region during the control time (cf. [1]). As proved in [7], [8] and [5], in $1-d$, this result holds under the sharp assumption that the coefficients belong to the class $B V(-1,1)$ of functions with bounded variation 
The extension of the GCC in [1 to some numerical approximation schemes for the transport and wave equations has been developed in the particular case of constant coefficients and uniform grids in [11, [12] and [35] by means of Fourier analysis.

The main goal of this paper is to rigorously construct characteristic and bi-characteristic rays for the finite difference numerical approximations of the transport and wave equations (1.1) and (1.4) on non-uniform meshes to describe how the energy of numerical solutions propagates.

1.2. Main results. In order to better describe the propagation properties of waves on non-uniform meshes, let us first introduce the grids and the numerical schemes we deal with. Let $h>0$ be the mesh size parameter, $g: \mathbb{R} \rightarrow \mathbb{R}$ be an increasing function on $\mathbb{R}, \mathcal{G}^{h}:=\left\{x_{j}:=j h, j \in \mathbb{Z}\right\}$ be the uniform grid of size $h$ of $\mathbb{R}$ and $\mathcal{G}_{g}^{h}:=\left\{g_{j}:=g\left(x_{j}\right), j \in \mathbb{Z}\right\}$ the non-uniform grid obtained by transforming the uniform one through the map $g$ (see Fig. 1). We also set $g_{j+1 / 2}:=g\left(x_{j+1 / 2}\right)$ to be the image through the map $g$ of the midpoints $x_{j+1 / 2}=(j+1 / 2) h, j \in \mathbb{Z}$. Along this paper, we focus on the case in which $g$ is regular. More precisely, we require $c_{g}:=c(g) / g^{\prime}$ to belong to $C^{0,1}(\mathbb{R})$, where $c(y):=1 / \varrho(y)$ and $c(y):=\sqrt{\sigma(y) / \rho(y)}$ for the transport/wave equations.

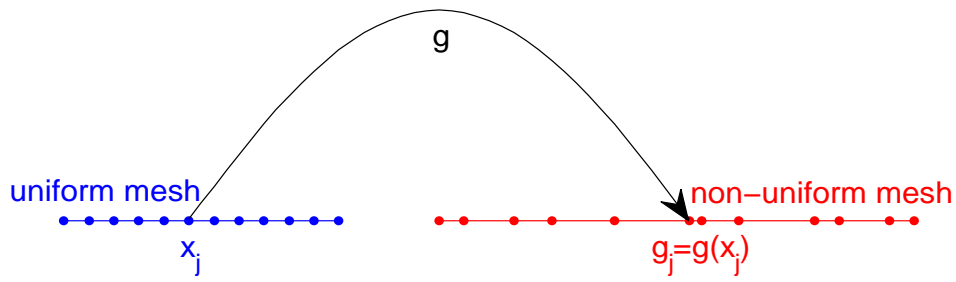

FiguRE 1. The smooth grid application $g$ transforms the nodes $x_{j}$ of the uniform grid $\mathcal{G}^{h}$ (blue) into nodes $g_{j}=g\left(x_{j}\right)$ of the non-uniform grid $\mathcal{G}_{g}^{h}$ (red).

We also denote by $\partial^{h} \mathbf{f}^{h}=\left(\partial^{h} f_{j}\right)_{j \in \mathbb{Z}}$ and $\partial^{h,{ }^{ \pm}} \mathbf{f}^{h}=\left(\partial^{h, \pm} f_{j}\right)_{j \in \mathbb{Z}}$ the first-order finite difference discrete derivatives on the uniform mesh, where $\partial^{h} f_{j}:=\left(f_{j+1}-f_{j-1}\right) / 2 h, \partial^{h, \pm} f_{j}:= \pm\left(f_{j \pm 1}-f_{j}\right) / h$ are the centered and the forward (+) / backward (-) finite differences.

1.2.1. The discrete transport equation. Along this paper, we consider the following finite difference semidiscretization of the transport equation (1.1):

$$
\varrho\left(g_{j}\right) \partial_{t} u_{j}(t)+\frac{\partial^{h} u_{j}(t)}{\partial^{h} g_{j}}=0, \quad u_{j}(0)=u_{j}^{0}
$$

on the non-uniform grid $\mathcal{G}_{g}^{h}$, where $j \in \mathbb{Z}$ and $t>0$. Here, $u_{j}(t)$ is an approximation of $u\left(g_{j}, t\right)$, where $u$ is the solution of the transport equation (1.1).

The total energy $\mathcal{E}_{\varrho, g}^{h}\left(\mathbf{u}^{h, 0}\right)$ below of the solution $\mathbf{u}^{h}(t)$ of $[1.6)$ is conserved in time

$$
\mathcal{E}_{\varrho, g}^{h}\left(\mathbf{u}^{h}(t)\right):=h \sum_{j \in \mathbb{R}} \varrho\left(g_{j}\right) \partial^{h} g_{j}\left|u_{j}(t)\right|^{2}=\mathcal{E}_{\varrho, g}^{h}\left(\mathbf{u}^{h, 0}\right) .
$$

1.2.2. The discrete wave equation. We also consider the finite difference approximation of the wave equation 1.4 on the non-uniform grid $\mathcal{G}_{g}^{h}$

$$
\rho\left(g_{j}\right) \partial_{t}^{2} u_{j}(t)-\frac{\left.\sigma\left(g_{j+1 / 2}\right) \frac{\partial^{h,+} u_{j}(t)}{\partial^{h,+} g_{j}}-\sigma\left(g_{j-1 / 2}\right)\right) \frac{\partial^{h,-} u_{j}(t)}{\partial^{h,-} g_{j}}}{h \partial^{h} g_{j}}=0, \quad u_{j}(0)=u_{j}^{0}, \partial_{t} u_{j}(0)=u_{j}^{1},
$$

where $j \in \mathbb{Z}$ and $t>0$. Here, $u_{j}(t)$ is an approximation of $u\left(g_{j}, t\right)$, where $u$ is the solution of the wave equation 1.4. The total energy $\mathcal{E}_{\rho, \sigma, g}^{h}\left(\mathbf{u}^{h}(t), \partial_{t} \mathbf{u}^{h}(t)\right)$ of the solution $\mathbf{u}^{h}(t)$ of 1.7 is conserved in time, i.e.,

$$
\mathcal{E}_{\rho, \sigma, g}^{h}\left(\mathbf{u}^{h}(t), \partial_{t} \mathbf{u}^{h}(t)\right):=\frac{h}{2} \sum_{j \in \mathbb{Z}}\left[\rho\left(g_{j}\right) \partial^{h} g_{j}\left|\partial_{t} u_{j}(t)\right|^{2}+\frac{\sigma\left(g_{j+1 / 2}\right)}{\partial^{h,+} g_{j}}\left|\partial^{h,+} u_{j}(t)\right|^{2}\right]=\mathcal{E}_{\rho, \sigma, g}^{h}\left(\mathbf{u}^{h, 0}, \mathbf{u}^{h, 1}\right) .
$$


1.2.3. The Wigner transform. The main tool for our theoretical analysis in both continuous and discrete settings is the so-called Wigner transform of pseudo-differential calculus introduced in 1932 by Eugene Wigner (cf. 34). For a precise definition of this notion, see [15, 21] and also Section 2 of this article. Roughly speaking, this is a quadratic transform used when the coefficients of the hyperbolic PDE under consideration vary at unit scale, while the wavelength of the initial data is asymptotically smaller. It allows to describe the propagation properties by capturing the characteristic rays of the model. A time dependent wave process like the solutions of the transport and of the wave equations (1.1), (1.4) and their finite difference semi-discretizations can be described either in terms of a wave function $u(y, t)$ at the point $y$ of the physical space or as the the bilinear product $u\left(y_{1}, t\right) \bar{u}\left(y_{2}, t\right)$ of the wave function and its conjugate at two points $y_{1}$ and $y_{2}$. In quantum dynamics, the scaled Fourier transform of this product with respect to the distance between the two points $y_{1}$ and $y_{2}$ is called Wigner transform. In the limit as the wavelength of the wave packet tends to zero, the Wigner transform becomes the so-called Wigner measure which can be seen as a particle density depending on the phase space variables $y$ (denoting the position) and $\xi$ (denoting the momentum). The corresponding Liouville equation for the Wigner measure is similar to a kinetic equation for real particles.

1.2.4. Short description of our main results. We now present in an itemized manner the main contributions of this paper.

- Principal symbols of the finite difference approximations on non-uniform meshes. It is well-known (cf. 1]) that the principal symbols of the continuous variable coefficients transport/wave equations (1.1) and (1.4) are given by

$$
\wp(y, t, \xi, \tau):=-\rho(y) \tau-\xi
$$

and, respectively,

$$
\wp(y, t, \xi, \tau):=-\rho(y) \tau^{2}+\sigma(y) \xi^{2} .
$$

These symbols are obtained by considering solutions of 1.1 or 1.4 arbitrarily concentrated in space around some point $y \in \mathbb{R}$ and oscillating at some frequencies $\tau$ and $\xi$ in time/space.

We prove that, for the discrete systems (1.6) and (1.7), the corresponding principal symbols are given by

and

$$
\wp(x, t, \xi, \tau):=-g^{\prime}(x) \rho(g(x)) \tau^{2}+4 \sin ^{2}\left(\frac{\xi}{2}\right) \frac{\sigma(g(x))}{g^{\prime}(x)}
$$

$$
\wp(x, t, \xi, \tau):=-g^{\prime}(x) \varrho(g(x)) \tau-\sin (\xi) .
$$

We note some changes of these discrete principal symbols with respect to the continuous ones $(1.8)$ and 1.9. Firstly, they depend on the space variable $x=g^{-1}(y)$ corresponding to the uniform grid. As a consequence, in this new space variable $x$, all the variable coefficients $\varrho, \rho$ and $\sigma$ have to be composed with $g$. Note also the appearance of $1 / g^{\prime}(x)$ accompanying each space derivative which is also due to the grid transformation $y=g(x)$. We note also that in (1.11) and 1.10 the Fourier symbols $\xi$ and $\xi^{2}$ of the first- and second-order space derivatives in the continuous symbols $(1.8)$ and $(1.9)$ have been replaced by the corresponding symbols $\sin (\xi)$ and $4 \sin ^{2}(\xi / 2)$ of the centered first-order finite difference $\partial^{h}$ and of the three-points finite difference approximation of the Laplacian.

- Propagation of the discrete Wigner measures. In Theorems 3.3 and 3.5 , we prove indeed that the expressions (1.11) and (1.10) are the appropriate ones. More precisely, we rigorously obtain the dynamics of the Wigner measures $\mathcal{W}$ for the solutions of the discrete transport and wave equations governed by Liouville equations of the form

$$
\mathcal{W}_{t}= \pm\left(c_{g}(x) \omega^{\prime}(\xi) \mathcal{W}_{x}-c_{g}^{\prime}(x) \omega(\xi) \mathcal{W}_{\xi}\right),
$$

where $c_{g}:=c(g) / g^{\prime}, c(y):=1 / \varrho(y)$ and $c(y):=\sqrt{\sigma(y) / \rho(y)}$ are the velocities corresponding to the continuous transport/wave equations and $\omega(\xi):=\sin (\xi)$ and $\omega(\xi):=2 \sin (\xi / 2)$ are the dispersion relations for the same discrete transport/wave equations on uniform meshes. These Liouville equations, being in particular transport equations, can be solved by the method of characteristics, yielding our precise 


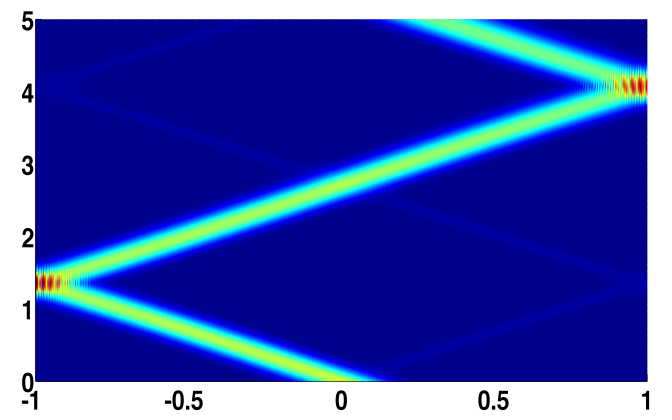

Figure 2. Behavior of the Gaussian wave packets for the finite difference scheme on nonuniform meshes at low frequencies.
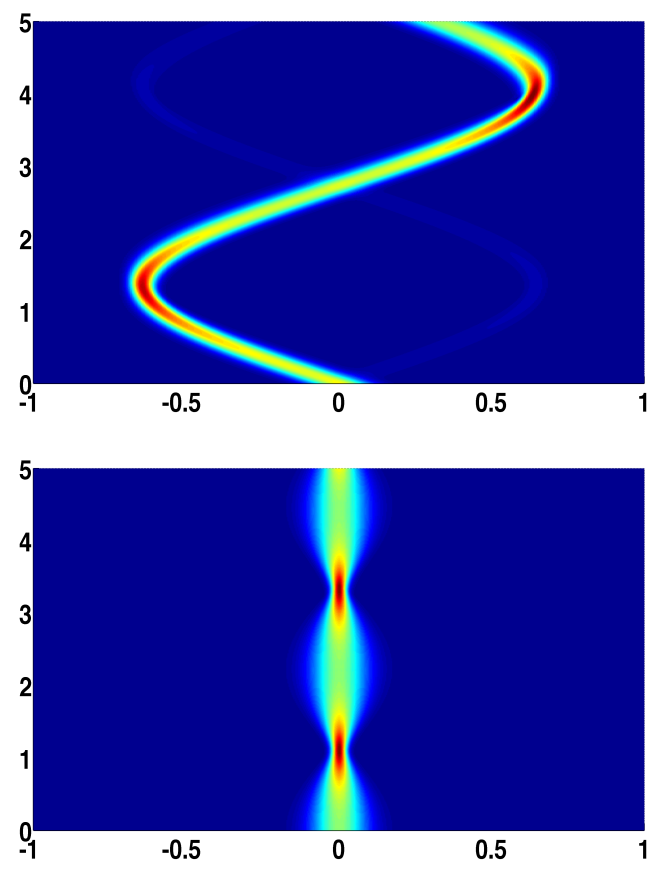
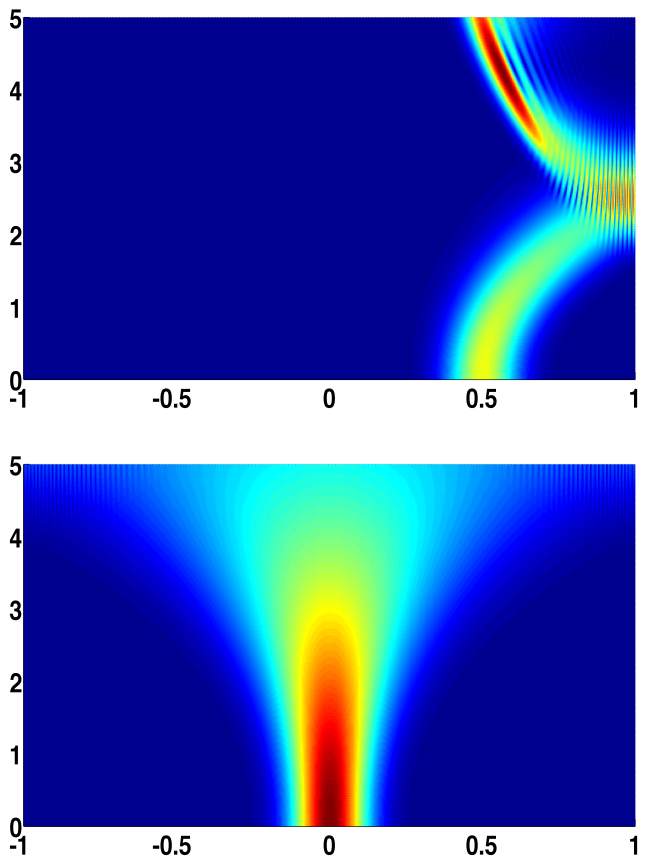

FiguRE 3. Behavior of the Gaussian wave packets for the finite difference scheme on nonuniform meshes at high frequencies.

definition of characteristic rays. Moreover, these characteristic rays coincides with those obtained by the resolution of the Hamiltonian systems associated to the principal symbols (1.11) and (1.10). Our results extend the ones by Macià in [22] dealing with numerical approximations of the wave equation with variable coefficients on uniform meshes and the ones by Markowich-Pietra-Pohl in [26] in which several discrete schemes for the Schrödinger equation on uniform meshes are analyzed.

- Qualitative analysis of the Hamiltonian systems describing the discrete characteristic rays. In Section 5 , we present several numerical simulations aimed to graphically confirm our theoretical results. More precisely, we consider approximations of both constant coefficients transport/wave equations on the bounded interval $(-1,1)$ and on two non-uniform meshes produced by the transformations $g^{1}(x)=\tan (\pi x / 4)$ and $g^{2}(x)=2 \sin (\pi x / 6)$, yielding a gradual refinement of the grid at the center $x=0$ of the space interval and, respectively, at the two endpoints. We also consider high-frequency oscillatory and concentrated 
Gaussian initial data. If at low frequencies the numerical solutions behave basically like the solution of the continuous model, i.e. they propagate along straight characteristic lines and reflect following the Snell law when they touch one of the two endpoints (see Fig. 2), when increasing the frequency, we encounter interesting phenomena like the curvature and the so-called umklapp or U-process (see [18, pp. 125), i.e. the reflection of waves before touching both endpoints due to the fact that the wave number of the numerical solution crosses the value $\pi$ and the group velocity of the numerical approximation changes the sign (Fig. 3. first row, left) or before touching one of the endpoints (Fig. 3. first row, right) or the one of stationary waves (see Fig. 3, second row). We will show that these pathologies can be explained by the behaviour of the corresponding phase portraits of the Hamiltonian systems yielding the characteristic rays. For example, the figures in the first/second column in Fig. 3 correspond to the grid transformations $g^{1}$ and $g^{2}$ and to center/saddle fixed points of the corresponding Hamiltonian systems.

The analysis in this paper is limited to the $1-d$ case, but the techniques we develop can also be used to analyze multi-dimensional problems.

1.3. Outline of the paper. In Section 2 we present some basic facts on the continuous Wigner transform, state the main existing results concerning the limit process in the Wigner transforms of the solutions of the continuous transport and wave equations (1.1) and (1.4) and describe the main ideas of the proofs that will help us to better understand the corresponding proofs in the discrete case. In Section 3 we introduce the numerical approximation schemes under consideration, the notion of discrete Wigner transform, some of its main properties and our main results, Theorems 3.3 and 3.5 . Section 4 is devoted to the proof of our two main results, Theorems 3.3 and 3.5 . In Section 5 we present some numerical simulations of high frequency wave packets and compare the numerical results to the ones predicted by our theoretical analysis. In Section 6 . we present the conclusions of the paper and list some related open problems. For the sake of completeness, in Appendix A we give the main steps for the proofs of the two convergence results, Propositions 3.1 and 3.4.

\section{Preliminaries on COntinuous models And Wigner transforms}

2.1. Bi-characteristic rays for the continuous transport and wave equations. a) Transport equation. Equation (1.3) can be also obtained by solving the Hamiltonian system of null bi-characteristic rays associated to the principal symbol $(1.8)$

$$
\left\{\begin{array}{l}
Y^{\prime}(s)=\partial_{\xi \wp}=-1, \quad t^{\prime}(s)=\partial_{\tau} \wp=-\rho(Y(s)), \\
\Xi^{\prime}(s)=-\partial_{y} \wp=\rho^{\prime}(Y(s)) \tau(s), \quad \tau^{\prime}(s)=-\partial_{t} \wp=0,
\end{array}\right.
$$

subjected to initial data $(Y(0), t(0), \Xi(0), \tau(0))=\left(y_{0}, 0, \xi_{0}, \tau_{0}\right)$ such that $\wp\left(y_{0}, 0, \xi_{0}, \tau_{0}\right)=0$. Let us denote by $(y(t), \xi(t))=(Y(s), \Xi(s))$ which solves the Hamiltonian system

$$
y^{\prime}(t)=c(y(t)), \quad \xi^{\prime}(t)=-c^{\prime}(y(t)) \xi(t), \quad y(0)=y_{0}, \xi(0)=\xi_{0} .
$$

Note that the first equation in 2.2 is precisely 1.3 .

b) Wave equation. For the wave equation (1.4), the null bi-characteristic rays associated to the principal symbol (1.9) are solutions of the following Hamiltonian system

$$
\left\{\begin{array}{l}
Y^{\prime}(s)=\partial_{\xi \wp}=2 \sigma(Y(s)) \Xi(s), \quad t^{\prime}(s)=\partial_{\tau \wp} \wp=-2 \rho(Y(s)) \tau(s), \\
\Xi^{\prime}(s)=-\partial_{y} \wp=\rho^{\prime}(Y(s)) \tau^{2}(s)-\sigma^{\prime}(Y(s)) \Xi^{2}(s), \quad \tau^{\prime}(s)=-\partial_{t} \wp=0,
\end{array}\right.
$$

subjected to initial data $(Y(0), t(0), \Xi(0), \tau(0))=\left(y_{0}, 0, \xi_{0}, \tau_{0}\right)$ such that $\wp\left(y_{0}, 0, \xi_{0}, \tau_{0}\right)=0$. Then $\tau(s)=\tau_{0}$ and $\wp(Y(s), t(s), \Xi(s), \tau(s))=0$ are constant in $s$. Let us denote by $\left(y^{ \pm}(t), \xi^{ \pm}(t)\right)=(Y(s), \Xi(s))$ the two families of solutions of 2.3 corresponding to one of the two possible roots $\tau_{0}^{ \pm}$of $\wp\left(Y(s), t(s), \Xi(s), \tau_{0}\right)=$ 0 given by $\tau_{0}^{ \pm}:= \pm \Xi(s) c(Y(s))$, with $c:=\sqrt{\sigma / \rho}$. Then $\left(y^{ \pm}(t), \xi^{ \pm}(t)\right)$ is the solution of the following Hamiltonian system of first-order ODEs in the time variable $t$ :

$$
\left(y^{ \pm}\right)^{\prime}(t)=\mp c\left(y^{ \pm}(t)\right), \quad\left(\xi^{ \pm}\right)^{\prime}(t)= \pm c^{\prime}\left(y^{ \pm}(t)\right) \xi^{ \pm}(t), \quad y^{ \pm}(0)=y_{0}, \quad \xi^{ \pm}(0)=\xi_{0} .
$$

Note that the first equation in 2.2 or 2.4 can be solved independently of the second one. As we will see, this is not the case for Hamiltonian systems corresponding to the numerical approximations for the wave and transport equations. 
When $c \equiv 1$, one can observe from $\sqrt{2.4}$ that, for each $\xi_{0}$, there are two characteristics going out from each point $y_{0}, y^{ \pm}(t)=y_{0} \mp t$. Moreover, if we consider the homogeneous Dirichlet boundary value problem for the wave equation (say, on the interval $(-1,1)$ ), each characteristic reaches the boundary in an uniform time not depending on the frequency $\xi_{0}$. This coincides with our intuition on the wave propagation and has several applications in control and inverse problems.

2.2. Continuous pseudo-differential operators and Wigner transforms. In the continuous setting, given a $d \times d$ - matrix valued function $\boldsymbol{\Theta}(y, \xi)$, we define the associated pseudo-differential operator $\boldsymbol{\Theta}\left(y, \epsilon \partial_{y}\right)$ by (cf. [15])

$$
\boldsymbol{\Theta}\left(y, \epsilon \partial_{y}\right) \mathbf{f}(y):=\frac{1}{2 \pi} \int_{\mathbb{R}} \Theta(y, \epsilon \xi) \widehat{\mathbf{f}}(\xi) \exp (i \xi y) d \xi
$$

where $\mathbf{f}(y)=\left(f_{1}(y), \cdots, f_{d}(y)\right)$ is a (column) vector-function and $\widehat{\mathbf{f}}(\xi)=\left(\widehat{f_{1}}(\xi), \cdots, \widehat{f}_{d}(\xi)\right)$ is the column vector containing the Fourier transforms of its components.

For $\epsilon>0$ and $\mathbf{f}^{1}(x)=\left(f_{1}^{1}(x), \cdots, f_{d}^{1}(x)\right)$ and $\mathbf{f}^{2}(x)=\left(f_{1}^{2}(x), \cdots, f_{d}^{2}(x)\right)$ being two (column) vectorvalued functions, we define the so-called Wigner transform matrix at scale $\epsilon$ of $\mathbf{f}^{1}$ and $\mathbf{f}^{2}$ as follows $(\mathbf{A} \otimes \mathbf{B}$ is the tensorial product of the two matrices $\mathbf{A}$ and $\mathbf{B}$, while $\mathbf{A}^{*}$ is the conjugate transpose of the matrix $\mathbf{A}$ ):

$$
\begin{aligned}
\mathcal{W}^{\epsilon}\left[\mathbf{f}^{1}, \mathbf{f}^{2}\right](x, \xi) & :=\frac{1}{2 \pi} \int_{\mathbb{R}} \mathbf{f}^{1}\left(x-\frac{\epsilon z}{2}\right) \otimes \mathbf{f}^{2, *}\left(x+\frac{\epsilon z}{2}\right) \exp (i \xi z) d z \\
& =\frac{1}{\epsilon} \frac{1}{(2 \pi)^{2}} \int_{\mathbb{R}} \widehat{\mathbf{f}}^{1}\left(\frac{\xi}{\epsilon}+\frac{\eta}{2}\right) \otimes \widehat{\mathbf{f}}^{2, *}\left(\frac{\xi}{\epsilon}-\frac{\eta}{2}\right) \exp (i \eta x) d \eta .
\end{aligned}
$$

Set $\mathcal{W}^{\epsilon}\left[\mathbf{f}^{1}\right]:=\mathcal{W}^{\epsilon}\left[\mathbf{f}^{1}, \mathbf{f}^{1}\right]$. When $d=1$, the tensorial product $\otimes$ becomes simply the multiplication of functions. In that case, we will remove the bold character of the arguments and of $\mathcal{W}$ and we will put $\mathcal{W}^{\epsilon}\left[f^{1}, f^{2}\right](x, \xi)$ for the scalar Wigner transform of two functions $f^{1}(x)$ and $f^{2}(x)$. Moreover, when the functions $\mathbf{f}^{1}$ and $\mathbf{f}^{2}$ depend also on the time variable $t$, we denote by $\mathcal{W}^{\epsilon}\left[\mathbf{f}^{1}, \mathbf{f}^{2}\right](x, t, \xi)$ the corresponding Wigner transform of $\mathbf{f}^{1}$ and $\mathbf{f}^{2}$.

Note the following two properties of the Wigner transform:

$$
\int_{\mathbb{R}} \mathcal{W}^{\epsilon}\left[\mathbf{f}^{1}, \mathbf{f}^{2}\right](x, \xi) d \xi=\mathbf{f}^{1}(x) \otimes \mathbf{f}^{2, *}(x), \int_{\mathbb{R}} \mathcal{W}^{\epsilon}\left[\mathbf{f}^{1}, \mathbf{f}^{2}\right](x, \xi) d x=\frac{1}{2 \pi \epsilon} \widehat{\mathbf{f}}^{1}\left(\frac{\xi}{\epsilon}\right) \otimes \widehat{\mathbf{f}}^{2, *}\left(\frac{\xi}{\epsilon}\right) .
$$

2.3. Existing results on the Wigner measure for the continuous transport equation (1.1). Set $w(y, t):=\sqrt{\varrho(y)} u(y, t)$, where $u$ is the solution of 1.1 . Then $w$ satisfies the transport equation

$$
\partial_{t} w(y, t)=-c(y) \partial_{y} w(y, t)-d(y) w(y, t), \quad w(y, 0)=w^{0}(y):=\sqrt{\varrho(y)} u^{0}(y), \quad y \in \mathbb{R}, t>0 .
$$

The coefficients $c$ and $d$ in 2.8 are given by

$$
c(y):=\frac{1}{\varrho(y)} \text { and } d(y):=\frac{1}{2}\left(\frac{1}{\varrho}\right)^{\prime}(y) .
$$

The $L^{2}(\mathbb{R})$-norm of $w$ is time conservative. For all $\epsilon>0$, any initial datum $u^{0}$ in $(1.1)$, for $w$ being the solution of $(2.8)$ with $w^{0}=\sqrt{\varrho} u^{0}$ and using property (2.7), we can express the time conservative total energy $\mathcal{E}_{\rho}(u(\cdot, t))$ in 11.2 of the solution $u$ of 1.1 in terms of the Wigner transform at scale $\epsilon$ of $w$ as follows:

$$
\mathcal{E}_{\rho}(u(\cdot, t))=\|w(\cdot, t)\|_{L^{2}(\mathbb{R})}^{2}=\int_{\mathbb{R}} \int_{\mathbb{R}} \mathcal{W}^{\epsilon}[w](x, t, \xi) d x d \xi .
$$

Using the arguments in [15, [17] or [21, the following result concerning the transport equation (2.8) can be proved (for the sake of completeness, we will give a sketch of this proof in Appendix A): 
Theorem 2.1. For any coefficient $c \in C^{0,1}(\mathbb{R})$ in (2.8) and any initial data $w^{0}=w^{\epsilon, 0}$ in (2.8) bounded in $L^{2}(\mathbb{R})$ as $\epsilon \rightarrow 0$, there exists a positive Radon measure $\mathcal{W}(y, t, \xi)$ defined on $\mathbb{R} \times \mathbb{R}_{+} \times \mathbb{R}$ such that, eventually after extracting subsequences, we get

$$
\mathcal{W}^{\epsilon}\left[w^{\epsilon}\right](y, t, \xi) \rightarrow \mathcal{W}(y, t, \xi) \text { weakly star in } \mathcal{S}^{\prime}\left(\mathbb{R}_{y} \times \mathbb{R}_{\xi}\right),
$$

uniformly on each compact set of the time interval $\mathbb{R}_{+}$. Moreover, the measure $\mathcal{W}$ satisfies the following Liouville equation:

$$
\partial_{t} \mathcal{W}(y, t, \xi)=-\mathrm{P}_{c, \xi} \mathcal{W}(y, t, \xi), \quad \mathcal{W}(y, 0, \xi)=\mathcal{W}^{0}(y, \xi):=\lim _{\epsilon \rightarrow 0} \mathcal{W}^{\epsilon}\left[w^{\epsilon, 0}\right](y, \xi) .
$$

For all $C^{0,1}$-functions $c=c(x)$ and $\omega=\omega(\xi)$, the operator $\mathrm{P}_{c, \omega}$ is defined as follows:

$$
\mathrm{P}_{c, \omega}:=c(x) \omega^{\prime}(\xi) \partial_{x}-c^{\prime}(x) \omega(\xi) \partial_{\xi} .
$$

2.4. Existing results on the Wigner measure of the continuous wave equation (1.4). For $u$ being the solution of $\sqrt{1.4}$, set $(w(y, t), \tilde{w}(y, t)):=\left(\sqrt{\rho(y)} \partial_{t} u(y, t), \sqrt{\sigma(y)} \partial_{y} u(y, t)\right)$. Then $(w, \tilde{w})\left(\right.$ whose $\left(L^{2}(\mathbb{R})\right)^{2}-$ norm is conserved in time) satisfies the system of first-order PDEs:

$$
\left\{\begin{array}{l}
\left(\begin{array}{c}
\partial_{t} w(y, t) \\
\partial_{t} \tilde{w}(y, t)
\end{array}\right)=\mathcal{A}\left(\begin{array}{c}
w(y, t) \\
\tilde{w}(y, t)
\end{array}\right), \forall y \in \mathbb{R}, t>0, \quad \mathcal{A}:=\left(\begin{array}{cc}
0 & c(y) \partial_{y}+d(y) \\
c(y) \partial_{y}+e(y) & 0
\end{array}\right) . \\
(w(y, 0), \tilde{w}(y, 0))=\left(w^{0}(y), \tilde{w}^{0}(y)\right):=\left(\sqrt{\rho}(y) u^{1}(y), \sqrt{\sigma}(y)\left(u^{0}\right)^{\prime}(y)\right)
\end{array}\right.
$$

The coefficients $c, d$ and $e$ are given by

$$
c(y):=\sqrt{\frac{\sigma(y)}{\rho(y)}}, \quad d(y):=\frac{\sigma^{\prime}(y)}{2 \sqrt{\sigma(y) \rho(y)}} \text { and } e(y):=-\frac{\sqrt{\sigma(y)} \rho^{\prime}(y)}{2 \rho(y) \sqrt{\rho(y)}},
$$

so that

$$
d(y)+e(y)=c^{\prime}(y) \text { and } d(y)-e(y)=\frac{(\sqrt{\rho \sigma})^{\prime}(y)}{\rho(y)} .
$$

Let $\mathbf{J}_{d}$ be the $d$-dimensional exchange matrix, i.e.,

$$
\mathbf{J}_{d}:=\left(\begin{array}{cccc}
0 & \cdots & 0 & 1 \\
0 & \cdots & 1 & 0 \\
\vdots & . \cdot & \vdots & \vdots \\
1 & \cdots & 0 & 0
\end{array}\right) .
$$

Since $\mathcal{A}^{*}=-\mathcal{A}+\left(d+e-c^{\prime}\right) \mathbf{J}_{2}$, the operator $\mathcal{A}$ in 2.14 is anti-self-adjoint if and only if $d+e=c^{\prime}$. This is precisely our case.

Let us introduce the following notation: $w^{ \pm}(y, t)=(w(y, t) \pm \tilde{w}(y, t)) / \sqrt{2}$, where $(w(y, t), \tilde{w}(y, t))$ is the solution of 2.14). Then the pair $\left(w^{+}(y, t), w^{-}(y, t)\right)$ solves the following coupled system of PDEs:

$$
\left\{\begin{array}{c}
\left(\begin{array}{c}
w_{t}^{+}(y, t) \\
w_{t}^{-}(y, t)
\end{array}\right)=\tilde{\mathcal{A}}\left(\begin{array}{c}
w^{+}(y, t) \\
w^{-}(y, t)
\end{array}\right), \text { with } \tilde{\mathcal{A}}:=\left(\begin{array}{cc}
c(y) \partial_{y}+\frac{1}{2} c^{\prime}(y) & -\frac{1}{2} \tilde{c}(y) \\
\frac{1}{2} \tilde{c}(y) & -c(y) \partial_{y}-\frac{1}{2} c^{\prime}(y)
\end{array}\right) \\
w^{ \pm}(y, 0)=w^{0, \pm}(y):=\frac{w^{0}(y) \pm \tilde{w}^{0}(y)}{\sqrt{2}} .
\end{array}\right.
$$

Here, $\tilde{c}(y):=d(y)-e(y)\left(d, e\right.$ as in 2.15). The operator $\tilde{\mathcal{A}}_{11}$ is precisely the one involved in the scalar transport equation 2.8) (with a different $c(:=\sqrt{\sigma / \rho})$ ), while $\tilde{\mathcal{A}}_{22}=-\tilde{\mathcal{A}}_{11}$. System 2.17) is similar to the one verified by $v_{n, \pm}$ in [14], (4.28), $\partial_{t} v_{n, \pm}= \pm i|\nabla| v_{h, \pm}$ for the constant coefficient multidimensional wave equation $(|\nabla|$ being the pseudo-differential operator generated by the symbol $|\xi|)$.

Set

$$
\mathcal{W}^{\epsilon, \pm}:=\mathcal{W}^{\epsilon}\left[w^{ \pm}\right] \text {and } \tilde{\mathcal{W}}^{\epsilon, \pm}:=\mathcal{W}^{\epsilon}\left[w^{+}, w^{-}\right] \pm \mathcal{W}^{\epsilon}\left[w^{-}, w^{+}\right]
$$

where $\mathcal{W}^{\epsilon}$ is the Wigner transform at scale $\epsilon$ in 2.6) and $w^{ \pm}$is the solution of 2.17). 
Note that, for all $\epsilon>0$, the time conservative energy $\mathcal{E}_{\varrho, \sigma}\left(u(\cdot, t), u_{t}(\cdot, t)\right)$ in 1.5 of the solution of the wave equation (1.4) admits the following equivalent representations in terms of the solution $(w, \widetilde{w})$ of (2.14), the solution $\left(w^{+}, w^{-}\right)$of 2.17$)$ and the Wigner transform of $\left(w^{+}, w^{-}\right)$:

$$
\begin{array}{r}
\mathcal{E}_{\varrho, \sigma}\left(u(\cdot, t), u_{t}(\cdot, t)\right)=\frac{1}{2} \int_{\mathbb{R}}\left(|w(y, t)|^{2}+|\widetilde{w}(y, t)|^{2}\right) d y=\frac{1}{2} \int_{\mathbb{R}}\left(\left|w^{+}(y, t)\right|^{2}+\left|w^{-}(y, t)\right|^{2}\right) d y \\
=\frac{1}{2} \int_{\mathbb{R}} \int_{\mathbb{R}} \operatorname{tr} \mathcal{W}^{\epsilon}\left[w^{+}, w^{-}\right](y, t, \xi) d y d \xi=\frac{1}{2} \int_{\mathbb{R}} \int_{\mathbb{R}}\left(\mathcal{W}^{\epsilon,+}(y, t, \xi)+\mathcal{W}^{\epsilon,-}(y, t, \xi)\right) d y d \xi .
\end{array}
$$

The following result explains the behavior of $\mathcal{W}^{\epsilon}:=\operatorname{tr} \mathcal{W}^{\epsilon}\left[w^{+}, w^{-}\right]$as $\epsilon \rightarrow 0$ :

Theorem 2.2. For any coefficient $c \in C^{0,1}(\mathbb{R})$ and any initial data $\left(w^{\epsilon, 0,+}, w^{\epsilon, 0,-}\right)$ in $(2.17)$ bounded in $\left(L^{2}(\mathbb{R})\right)^{2}$ as $\epsilon \rightarrow 0$, there exists a positive Radon measure $\mathcal{W}(y, t, \xi)$ defined on $\mathbb{R} \times \mathbb{R}_{+} \times \mathbb{R}$ such that, eventually after extracting subsequences, 2.11) holds uniformly on each compact set of the time interval $\mathbb{R}_{+}$.

Moreover, $\mathcal{W}$ can be split into two positive Radon measures as $\mathcal{W}=\mathcal{W}^{+}+\mathcal{W}^{-}$, where $\mathcal{W}^{ \pm}:=$ $\lim _{\epsilon \rightarrow 0} \mathcal{W}^{\epsilon, \pm}$ is the Wigner measures of $w^{\epsilon, \pm}$ solving (2.17) with initial data $w^{\epsilon, 0, \pm}$. Each Wigner measure $\mathcal{W}^{ \pm}$satisfies the Liouville equation

$$
\partial_{t} \mathcal{W}^{ \pm}(y, t, \xi)= \pm \mathrm{P}_{c, \xi} \mathcal{W}^{ \pm}(y, t, \xi), \quad \mathcal{W}^{ \pm}(y, 0, \xi)=\lim _{\epsilon \rightarrow 0} \mathcal{W}^{\epsilon, \pm}(y, 0, \xi), \quad y, \xi \in \mathbb{R}, t>0
$$

where the operator $\mathrm{P}_{c, \xi}$ is as in 2.13) with $\omega(\xi)=\xi$.

2.5. Concentrated and oscillatory initial data. The most singular initial data for the transport and wave equations (1.1) and (1.4) are the highly concentrated and oscillatory ones, i.e.,

$$
u^{0}(y)=u^{\epsilon, 0}(y):=\epsilon^{\alpha} f\left(\frac{y-y_{0}}{\epsilon^{\beta}}\right) \exp \left(\frac{i y \xi_{0}}{\epsilon}\right), \quad 2 \alpha+\beta=0,0<\beta<1,
$$

with $f \in L^{2}(\mathbb{R})$ for the transport equation, and

$$
u^{0}(y)=u^{\epsilon, 0}(y):=\epsilon^{\alpha} f\left(\frac{y-y_{0}}{\epsilon^{\beta}}\right) \exp \left(\frac{i y \xi_{0}}{\epsilon}\right), u^{1}=u^{\epsilon, 1}:=c u_{y}^{\epsilon, 0}, \quad 2(\alpha-1)+\beta=0,0<\beta<1,
$$

with $f \in H^{1}(\mathbb{R}),\left(y_{0}, \xi_{0}\right) \in \mathbb{R}^{2}, \xi_{0} \neq 0$ and $c$ as in 2.15 for the wave equation (see 24 for $\beta=1 / 2$ ).

The role of the scaling factors $\epsilon^{\alpha}$ in 2.22 (and similarly in (2.21)) is to make the total energy $\mathcal{E}_{\rho, \sigma}\left(u^{\epsilon, 0}, u^{\epsilon, 1}\right)$ to be bounded as $\epsilon \rightarrow 0$. These initial data are concentrated at scale $\epsilon^{-\beta}$ through the factor $f\left(\left(y-y_{0}\right) \epsilon^{-\beta}\right)$ around the point $y_{0}$ in space and oscillate at wavelength $\epsilon$ in the direction $\xi_{0}$. The first condition $2(\alpha-1)+\beta=0$ on the exponents $\alpha$ and $\beta$ is to guarantee that the total energy of the initial data is uniformly bounded as $\epsilon \rightarrow 0$. Indeed,

$$
\mathcal{E}_{\rho, \sigma}\left(u^{\epsilon, 0}, u^{\epsilon, 1}\right)=\left\|\sqrt{\sigma} u_{y}^{\epsilon, 0}\right\|_{L^{2}}^{2} \sim \epsilon^{2 \alpha-\beta}\left\|f^{\prime}\right\|_{L^{2}}^{2}+\epsilon^{2(\alpha-1)+\beta} \xi_{0}^{2}\|f\|_{L^{2}}^{2} .
$$

Moreover, for this total energy to be of order $O(1)$, there are two options: i) $2 \alpha-\beta=0$ and $2(\alpha-1)+\beta \geq 0$ or ii) $2 \alpha-\beta \geq 0$ and $2(\alpha-1)+\beta=0$. In both cases, $\alpha \geq 1 / 2$, while $\beta \geq 1$ and $\beta \leq 1$ for the first/second inequalities system. The fact that $\beta>0$ is needed to obtain concentrated initial data. We can exclude the case $\beta \geq 1$ since we want the semiclassical measure $\mathcal{W}^{+}(y, 0, \xi)$ of $w^{\epsilon, 0,+}=\left(\sqrt{\sigma} u_{y}^{\epsilon, 0}+\sqrt{\rho} u^{\epsilon, 1}\right) / \sqrt{2}=\sqrt{2 \sigma} u_{y}^{\epsilon, 0}$ to be $\delta_{y_{0}}(y) \otimes \delta_{\xi_{0}}(\xi)$ (modulo a multiplicative constant). More precisely, for the choice 2.22 of the initial data $\left(u^{0}, u^{1}\right)=\left(u^{\epsilon, 0}, u^{\epsilon, 1}\right)$ in 1.4 , we can prove that

$$
\mathcal{W}^{+}(y, 0, \xi)=2 \sigma\left(y_{0}\right) \xi_{0}^{2}\|f\|_{L^{2}}^{2} \delta_{y_{0}}(y) \otimes \delta_{\xi_{0}}(\xi)
$$

Consequently, at future times $t>0$, the Wigner measure $\mathcal{W}^{+}$which is the solution of the transport equation 2.20 in phase-space takes the explicit form $\mathcal{W}^{+}(y, t, \xi)=2 \sigma\left(y_{0}\right) \xi_{0}^{2}\|f\|_{L^{2}}^{2} \delta_{y^{-}(t)}(y) \otimes \delta_{\xi^{-}(t)}(\xi)$ and propagates along the characteristics $\left(y^{-}(t), \xi^{-}(t)\right)$ being the solution of the ODE system (2.4) corresponding to the initial data $\left(y_{0}, \xi_{0}\right)$. For the same initial data $(2.22)$ in $(1.4), \mathcal{W}^{-}(y, 0, \xi)$ in 2.20 vanishes. Note that if $f$ in 2.21$)$ or 2.22 is compact supported, then the support of order $\epsilon^{\beta}$ of the envelope $f\left(\left(y-y_{0}\right) / \epsilon^{\beta}\right)$ is asymptotically larger as $\epsilon \rightarrow 0$ than the wavelength of order $\epsilon$ of the data $u^{\epsilon, 0}$ when $\beta<1$. 
2.6. Interpretation of Theorems 2.1 and 2.2 in terms of solutions of the original transport and wave equations (1.1) and (1.4). The corresponding solution of $\sqrt{1.1}$ ) is driven by characteristics, so that if the initial datum $u^{0}=u^{\epsilon, 0}$ is concentrated at some point, the solution $u^{\epsilon}(\cdot, t)$ is necessarily concentrated at any further time $t>0$ for all $\epsilon>0$. However, the analysis of the concentration of energy for the solutions of the second-order wave equation is more subtle. More precisely, the energy density $d\left[u^{\epsilon}, u_{t}^{\epsilon}\right]$ corresponding to initial data $\left(u^{\epsilon, 0}, u^{\epsilon, 1}\right)$ as in 2.22 propagates along the space component $y^{-}(t)$ of the solution $\left(y^{-}(t), \xi^{-}(t)\right)$ of (2.4) starting at $\left(y_{0}, \xi_{0}\right)$, in the sense that

$$
\lim _{\epsilon \rightarrow 0} \int_{D_{r}(t)} d\left[u^{\epsilon}, u_{t}^{\epsilon}\right](y, t) d y=0, \quad d\left[u, u_{t}\right](y, t):=\frac{1}{2}\left(\varrho(y)\left|u_{t}(y, t)\right|^{2}+\sigma(y)\left|u_{y}(y, t)\right|^{2}\right)
$$

for all $r>0$, with $D_{r}(t):=\mathbb{R} \backslash B\left(y^{-}(t), r\right)$ and $B(x, r):=(x-r, x+r)$. Indeed, using the properties (2.7) of the Wigner transform, we obtain $\left(D_{R, r}(t):=B(0, R) \backslash B\left(y^{-}(t), r\right), D_{R}=\mathbb{R} \backslash B(0, R)\right)$ :

$$
\begin{aligned}
\int_{D_{r}(t)} d\left[u^{\epsilon}, u_{t}^{\epsilon}\right](y, t) d y & =A_{R}^{\epsilon}+\frac{1}{2} \int_{D_{R, r}(t)}\left(\left|w^{\epsilon,+}(y, t)\right|^{2}+\left|w^{\epsilon,-}(y, t)\right|^{2}\right) d y \\
& =A_{R}^{\epsilon}+\frac{1}{2} \int_{D_{R, r}(t)} \int_{\mathbb{R}}\left(\mathcal{W}^{\epsilon,+}(y, t, \xi)+\mathcal{W}^{\epsilon,-}(y, t, \xi)\right) d \xi d y=A_{R}^{\epsilon}+B_{R, r}^{\epsilon}+C_{R, r}^{\epsilon} .
\end{aligned}
$$

Set $\chi_{A}^{\delta}$ a regularization of size $\delta$ of the characteristic function of $A, \chi_{A}$. The regularization parameter $\delta$ is chosen such that $\chi_{D_{R, r}(t)}^{\delta}\left(y^{-}(t)\right)=0$. In the above identity, $A_{R}^{\epsilon}, B_{R, r}^{\epsilon}$ and $C_{R, r}^{\epsilon}$ are given by

$$
\begin{gathered}
A_{R}^{\epsilon}:=\frac{1}{2} \int_{D_{R}}\left(\left|w^{\epsilon,+}(y, t)\right|^{2}+\left|w^{\epsilon,-}(y, t)\right|^{2}\right) d y, \\
B_{R, r}^{\epsilon}:=\frac{1}{2} \int_{\mathbb{R}} \int_{\mathbb{R}} \chi_{D_{R, r}(t)}^{\delta}(y) \chi_{B(0, R)}^{\delta}(\xi)\left(\mathcal{W}^{\epsilon,+}(y, t, \xi)+\mathcal{W}^{\epsilon,-}(y, t, \xi)\right) d \xi d y, \\
C_{R, r}^{\epsilon}:=\frac{1}{2} \int_{\mathbb{R}} \int_{\mathbb{R}} \chi_{D_{R, r}(t)}^{\delta}(y)\left(1-\chi_{B(0, R)}^{\delta}(\xi)\right)\left(\mathcal{W}^{\epsilon,+}(y, t, \xi)+\mathcal{W}^{\epsilon,-}(y, t, \xi)\right) d \xi d y .
\end{gathered}
$$

For any $R>\left|y^{-}(t)\right|$, using $\sqrt{2.23}$, the fact that $W^{-} \equiv 0$ and that $\chi_{D_{R, r}(t)}^{\delta}\left(y^{-}(t)\right)=0$, we obtain that $B_{R, r}^{\epsilon} \rightarrow 0$ as $\epsilon \rightarrow 0$. Since $w^{\epsilon, \pm}$ belongs to $L^{2}(\mathbb{R})$ uniformly as $\epsilon \rightarrow 0$, then the sequences $w^{\epsilon, \pm}$ are compact at infinity in the sense that

$$
\limsup _{\epsilon \rightarrow 0} \int_{|y|>R}\left|w^{\epsilon, \pm}(y, t)\right|^{2} d y \rightarrow 0 \text { as } R \rightarrow \infty,
$$

so that for each $\epsilon$, there exists $R$ large enough such that $A_{R}^{\epsilon}$ is as small as one wants.

\section{Main Results AND BIBliographical COMments}

Notation 1. By lowercase letters accompanied by the superscript $h\left(\right.$ e.g. $\left.\mathbf{f}^{h}\right)$ we denote an infinite column vector associating to each node $x_{j}$ of the uniform grid $\mathcal{G}^{h}$ an unique value $f_{j}$, while $\mathbf{f}^{\mathbf{h}}$ associates to each $x_{j}$ a d-dimensional vector of values $\mathbf{f}_{j}=\left(f_{j, 1}, \cdots, f_{j, d}\right)$.

3.1. Discrete pseudo-differential operators and Wigner transforms. Let us start this section by introducing the notions of discrete pseudo-differential operator and Wigner transform that will be systematically used along this section and in Section 4.

For a $d \times d$ - matrix valued function $\boldsymbol{\Theta}=\boldsymbol{\Theta}(x, \xi)$, let us introduce the discrete pseudo-differential operator $\Theta^{h}\left(x, h \partial_{x}\right)$ by (cf. [27])

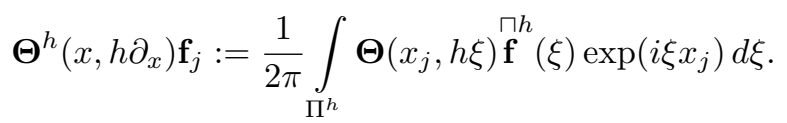


Here, $\mathbf{f}^{\mathbf{h}}:=\left(\mathbf{f}_{j}\right)_{j \in \mathbb{Z}}$, with $\mathbf{f}_{j}=\left(f_{j}^{1}, \cdots, f_{j}^{d}\right), \Pi^{h}:=[-\pi / h, \pi / h]$ and $\mathbf{f}^{h}(\xi)=(\stackrel{\sqcap h, k}{f}(\xi))_{1 \leq k \leq d}$ being the column vector containing the semi-discrete Fourier transforms (SDFT) $f^{h, k}(\xi)$ at scale $h$ of the components of

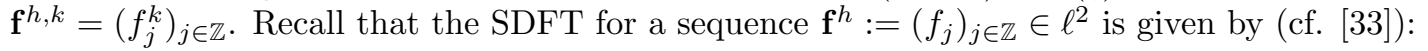

$$
\stackrel{\sqcap h}{f}^{h}(\xi):=h \sum_{j \in \mathbb{Z}} f_{j} \exp \left(-i \xi x_{j}\right) \quad \forall \xi \in \Pi^{h} .
$$

When $\boldsymbol{\Theta}(x, \xi)=\mathbf{A}(x) \mathbf{B}(\xi)$ is a matrix in separated variables, we denote by $\boldsymbol{\Theta}_{\mathbf{A}, \mathbf{B}}^{h}$ the corresponding pseudo-differential operator.

We define the discrete Wigner transform matrix at scale $\epsilon$ to be (see e.g. [22] or [23]):

$$
\mathcal{W}^{\epsilon}\left[\mathbf{f}^{\mathbf{h}, 1}, \mathbf{f}^{\mathbf{h}, 2}\right]\left(\frac{x_{m}}{2}, \xi\right):=\frac{h}{2 \pi \epsilon} \sum_{n \equiv m} \mathbf{f}_{\frac{m}{2}-\frac{n}{2}}^{1} \otimes\left(\mathbf{f}_{\frac{m}{2}+\frac{n}{2}}^{2}\right)^{*} \exp \left(\frac{i x_{n} \xi}{\epsilon}\right)
$$

where $\mathbf{f}^{\mathbf{h}, 1}, \mathbf{f}^{\mathbf{h}, 2}$ are two discrete functions associating to each grid point $x_{j}$ the $d$-dimensional vectors $\mathbf{f}_{j}^{1}$ and $\mathbf{f}_{j}^{2}$ and $\xi \in[-\epsilon \pi / h, \epsilon \pi / h]$. Here and in the sequel, $\equiv$ designs the equivalence modulo 2 . In this paper, we restrict the analysis to the cases $d=1$ and $d=2$ (corresponding to the discrete $1-d$ transport/wave equation). When $d=1$, the tensor product on the right hand side of $(3.3)$ is simply the scalar multiplication. To emphasize the fact that, for $d=1, \mathcal{W}^{\epsilon}\left[\mathbf{f}^{\mathbf{h}, 1}, \mathbf{f}^{\mathbf{h}, 2}\right]$ is a scalar quantity, we will replace the bold symbol in $\mathcal{W}^{h}$ by $\mathcal{W}^{h}$ and denote by $\mathcal{W}^{\epsilon}\left[\mathbf{f}^{h, 1}, \mathbf{f}^{h, 2}\right]$ the scalar discrete Wigner transform. We also set $\mathcal{W}^{\epsilon}\left[\mathbf{f}^{\mathbf{h}}\right]:=\mathcal{W}^{\epsilon}\left[\mathbf{f}^{\mathbf{h}}, \mathbf{f}^{\mathbf{h}}\right]$.

The analysis in this paper is restricted to the discrete solutions corresponding to the highest frequencies for which $\epsilon=h, \xi \in[-\pi, \pi]$ and $\exp \left(i x_{n} \xi / \epsilon\right)=\exp (i n \xi)$. Observe that when $m$ is even, $\mathcal{W}^{h}\left[\mathbf{f}^{\mathbf{h}, 1}, \mathbf{f}^{\mathbf{h}, 2}\right]\left(x_{m} / 2, \cdot\right)$ is a $\pi$-periodic function in $\xi$, while when $m$ is odd, $\mathcal{W}^{h}\left[\mathbf{f}^{\mathbf{h}, 1}, \mathbf{f}^{\mathbf{h}, 2}\right]\left(x_{m} / 2, \cdot\right)$ is $2 \pi$ periodic in $\xi$. Thus, for any value of $m \in \mathbb{Z}, \mathcal{W}^{h}\left[\mathbf{f}^{\mathbf{h}, 1}, \mathbf{f}^{\mathbf{h}, 2}\right]\left(x_{m} / 2, \cdot\right)$ is $2 \pi$-periodic. This justifies the fact that, for the discrete case, $[-\pi, \pi]$ is the suitable choice for the domain of the phase variable $\xi$.

Notation 2. Let us introduce the Hilbert space $\ell^{2}$ of square summable discrete functions with inner product given by $\left(\mathbf{f}^{h, 1}, \mathbf{f}^{h, 2}\right)_{\ell^{2}}:=h \sum_{j \in \mathbb{Z}} f_{j}^{1} \bar{f}_{j}^{2}$ and $\|\cdot\|_{\ell^{2}}$ be the corresponding norm. The space $\dot{\hbar}^{1}$ contains the discrete functions $\mathbf{f}^{h}$ such that $\partial^{h,+} \mathbf{f}^{h} \in \ell^{2}$ and is endowed with the inner product $\left(\mathbf{f}^{h, 1}, \mathbf{f}^{h, 2}\right)_{\dot{\hbar}^{1}}:=$ $\left(\partial^{h,+} \mathbf{f}^{h, 1}, \partial^{h,+} \mathbf{f}^{h, 2}\right)_{\ell^{2}}$ and the norm $\|\cdot\|_{\dot{\hbar}^{1}}$. We also employ the notation $\mathbf{x}^{h}:=\left(x_{j}\right)_{j \in \mathbb{Z}}$ and $f\left(\mathbf{x}^{h}\right):=$ $\left(f\left(x_{j}\right)\right)_{j \in \mathbb{Z}}$, for any continuous function $f$.

3.2. The discrete transport equation. The finite difference numerical scheme (1.6) approximating the transport equation (1.1) converges in the classical sense of numerical analysis. More precisely, the following convergence result (whose proof is provided in Appendix A holds:

Proposition 3.1. Assume that $\varrho \in C^{1}(\mathbb{R})$ in $\left.\sqrt{1.1}\right)$ is such that there exist $\varrho^{ \pm}$, $\varrho_{d}^{+}$so that $0<\varrho^{-} \leq \varrho(y) \leq \varrho^{+}$ and $\left|\varrho^{\prime}(y)\right| \leq \varrho_{d}^{+}$for all $y \in \mathbb{R}$ and that the initial datum $u^{0}$ belongs to $C_{c}^{2}(\mathbb{R})$. We also consider quasi-uniform grids, i.e. non-uniform grids $\mathcal{G}_{g}^{h}$ given by $g \in C^{1}(\mathbb{R})$ such that there exist $g_{d}^{ \pm}$so that $0<g_{d}^{-} \leq\left|g^{\prime}(x)\right| \leq g_{d}^{+}$, for all $x \in \mathbb{R}$. Under these hypotheses, the numerical approximation scheme (1.6) with initial data $\mathbf{u}^{h, 0}:=$ $\left(u^{0}\left(g_{j}\right)\right)_{j \in \mathbb{Z}}$ converges with order $O(h)$ to the solution of the transport equation (1.1) in the $\ell^{2}$-norm, i.e. there exists a constant $C\left(t, g, \varrho, u^{0}\right)$ independent of $h$ such that

$$
\left\|\mathbf{u}^{h}(t)-u\left(g\left(\mathbf{x}^{h}\right), t\right)\right\|_{\ell^{2}} \leq h C\left(t, g, \varrho, u^{0}\right) .
$$

The function $v(x, t):=u(g(x), t)$, where $u(y, t)$ is the solution of $(1.1)$, solves the transport equation

$$
g^{\prime}(x) \varrho(g(x)) v_{t}(x, t)+v_{x}(x, t)=0, \quad v(x, 0)=u(g(x), 0)=u^{0}(g(x)), \quad x \in \mathbb{R}, t>0 .
$$

Thus, by reconsidering the values $u_{j}(t)$ associated initially to the points of the non-uniform grid as being values $v_{j}(t)=u_{j}(t)$ at points of the uniform grid, we obtain that $v_{j}(t)$ is the solution of the following finite difference scheme for the transport equation 3.5 on the uniform grid $\mathcal{G}^{h}$ :

$$
\partial^{h} g_{j} \varrho\left(g_{j}\right) \partial_{t} v_{j}(t)+\partial^{h} v_{j}(t)=0, \quad v_{j}(0)=v_{j}^{0}=u_{j}^{0}, j \in \mathbb{Z} .
$$


Indeed, from Proposition 3.1 we see that $\mathbf{v}^{h}(t) \sim v\left(\mathbf{x}^{h}, t\right)$, where $v(x, t)$ and $\left.\mathbf{v}^{h}(t)\right)$ are the solutions of (3.5) and (3.6). This idea of reorganizing numerical schemes on non-uniform meshes in order to obtain approximations of PDEs of the same type with different variable coefficients on uniform meshes is not new and it has been used for example in [30. to construct preconditioning strategies for systems arising from discretizations of elliptic PDEs on non-uniform meshes based on known algorithms for approximating PDEs with variable coefficients on uniform meshes.

The null bi-characteristic lines associated to the principal symbol (1.11) of the discrete transport equation (3.6) are the solutions of the following system:

$$
\left\{\begin{array}{l}
X^{\prime}(s)=\partial_{\xi \wp}=-\cos (\Xi(s)), t^{\prime}(s)=\partial_{\tau} \wp=-g^{\prime}(X(s)) \varrho(g(X(s))), \\
\Xi^{\prime}(s)=-\partial_{x} \wp=\tau\left(g^{\prime}(\cdot) \varrho(g(\cdot))\right)^{\prime}(X(s)), \tau^{\prime}(s)=-\partial_{t} \wp=0,
\end{array}\right.
$$

subjected to the initial data $(X(0), t(0), \Xi(0), \tau(0))=\left(x_{0}, 0, \xi_{0}, \tau_{0}\right)$ such that $\wp\left(x_{0}, 0, \xi_{0}, \tau_{0}\right)=0$. Then $\tau(s)=\tau_{0}$ and $\wp(X(s), t(s), \Xi(s), \tau(s))=0$ are conserved in $s$. Set $(x(t), \xi(t)):=(X(s), \Xi(s))$ to be the solutions of (3.7) as functions of $t$. Then $(x(t), \xi(t))$ is the solution of the following Hamiltonian system:

$$
x^{\prime}(t)=c_{g}(x(t)) \cos (\xi(t)), \xi^{\prime}(t)=-c_{g}^{\prime}(x(t)) \sin (\xi(t)), x(0)=x_{0}, \xi(0)=\xi_{0}, c_{g}(x):=\frac{1}{\left(g^{\prime}(x) \varrho(g(x))\right.} .
$$

The characteristic rays on the non-uniform grid $\mathcal{G}_{g}^{h}$ are the curves $(y(t):=g(x(t)), \xi(t))$, with $(x(t), \xi(t))$ being the solution of $(3.8)$. Therefore, $(y(t), \xi(t))$ is the solution of the non-Hamiltonian system below, with $c$ as in 2.9$)$ and $c_{g}$ as in 3.8 , but for which the quantity $\sin (\xi(t)) / c_{g}\left(g^{-1}(y(t))\right)$ is time conservative:

$$
y^{\prime}(t)=c(y(t)) \cos (\xi(t)), \xi^{\prime}(t)=-c_{g}^{\prime}\left(g^{-1}(y(t))\right) \sin (\xi(t)), y(0)=y_{0}:=g\left(x_{0}\right), \xi(0)=\xi_{0} .
$$

As in the continuous case, we will not apply the discrete Wigner transform directly on the solution $v_{j}$ of (3.6), but on $w_{j}:=v_{j} \sqrt{\partial^{h} g_{j} \rho\left(g_{j}\right)}$ whose $\ell^{2}$-norm is conserved in time.

Lemma 3.2. For the solution $\mathbf{v}^{h}(t)$ of (3.6), set $w_{j}(t):=v_{j}(t) \sqrt{\partial^{h} g_{j} \rho\left(g_{j}\right)}$. The discrete function $\mathbf{w}^{h}(t)$ conserves its $\ell^{2}$-norm and is the solution of the system

$$
\partial_{t} w_{j}(t)=-\gamma_{j} \partial^{h} w_{j}(t)-\frac{1}{2} \delta_{j}\left(w_{j+1}(t)+w_{j-1}(t)\right), \quad w_{j}(0)=\frac{1}{\alpha_{j}} v_{j}^{0}:=\frac{1}{\alpha_{j}} u_{j}^{0}, j \in \mathbb{Z} .
$$

Here, $\gamma_{j}:=\gamma^{h}\left(x_{j}\right)$ and $\delta_{j}:=\delta^{h}\left(x_{j}\right)$, where

$$
\gamma^{h}(x):=\frac{\alpha^{h}(x)\left(\alpha^{h}(x+h)+\alpha^{h}(x-h)\right)}{2}, \delta^{h}(x):=\alpha^{h}(x) \partial^{h} \alpha^{h}(x) \text { and } \alpha^{h}(x):=\frac{1}{\sqrt{\partial^{h} g(x) \varrho(g(x))}} .
$$

Remark that the following approximations hold:

$$
\gamma^{h}(x) \sim c_{g}(x), \delta^{h}(x) \sim \frac{1}{2} c_{g}^{\prime}(x) \text { and } \alpha^{h}(x) \sim \sqrt{c_{g}(x)} \text { as } h \rightarrow 0, \text { with } c_{g}(x):=\frac{1}{\varrho(g(x)) g^{\prime}(x)} .
$$

Proof of Lemma 3.2. By replacing $v_{j}(t)=\alpha_{j} w_{j}(t)$ in 3.6. (which can be written as $\partial_{t} v_{j}(t) / \alpha_{j}^{2}+\partial^{h} v_{j}(t)=$ $0)$, we obtain

$$
\partial_{t} w_{j}(t)+\frac{\alpha_{j+1} \alpha_{j} w_{j+1}(t)-\alpha_{j} \alpha_{j-1} w_{j-1}(t)}{2 h}=0 .
$$

But (3.13) can be reorganized as (3.10) using the obvious identity $a b-c d=(a+c)(b-d) / 2+(a-c)(b+d) / 2$ in the particular case $a=\alpha_{j+1} \alpha_{j}, b=w_{j+1}(t), c=\alpha_{j} \alpha_{j-1}$ and $d=w_{j-1}(t)$.

System (3.10) is just a finite difference discrete analogue of 2.8$)$ on the uniform mesh $\mathcal{G}^{h}$ in which $c(y)$ is replaced by $c_{g}(x)$. From now on, unless stated, we will use only the discrete transport equation (3.10 since it is the one conserving the $\ell^{2}$-norm of the numerical solution. As we will see, the Wigner measure $\mathcal{W}:=\lim _{h \rightarrow 0} \mathcal{W}^{h}\left[\mathbf{w}^{h}\right]$ of the solution $\mathbf{w}^{h}(t)$ propagates along the bi-characteristic curves $(x(t), \xi(t))$ described by (3.8). Having the equation (3.10) defined on the uniform grid $\mathcal{G}^{h}$ has the advantage that, using the results in [23, we know how to pass to the limit in the Wigner transform of the initial data $\mathbf{w}^{h, 0}:=\mathbf{w}^{h}(0)$. For instance, when $\mathbf{w}^{h, 0}$ is a sampling of $u^{\epsilon, 0}$ in 2.21 (thus, $\alpha=-\beta / 2$ and any $\beta \in(0,1)$ ) on the uniform grid $\mathcal{G}^{h}$, then the corresponding Wigner measure is $\mathcal{W}(x, 0, \xi)=\delta_{y_{0}}(x) \otimes \delta_{\xi_{0}}(\xi)$. 
Let $\lambda_{c, \omega}(x, \xi)=c(x) \omega(\xi)$ be a scalar function in separated variables. Using the notation for pseudodifferential operators in Subsection 3.1, the discrete transport equation 3.10 can be written as

$$
\partial_{t} \mathbf{w}^{h}(t)=-\frac{i}{h} \lambda_{\gamma^{h}, \omega}^{h}\left(x, h \partial_{x}\right) \mathbf{w}^{h}(t)-\lambda_{\delta^{h}, \omega^{\prime}}^{h}\left(x, h \partial_{x}\right) \mathbf{w}^{h}(t), \text { with } \omega(\xi)=\sin (\xi)
$$

Our first result (to be proved in Section 4 describes the asymptotic behavior as $h \rightarrow 0$ of the discrete Wigner transform $\mathcal{W}^{h}\left[\mathbf{w}^{h}\right]$, where $\mathbf{w}^{h}(t)$ is the solution of the transport equation 3.10.

Theorem 3.3. For any coefficient $\varrho$ in 1 1.1, any non-uniform grid $\mathcal{G}_{g}^{h}$ obtained by a transformation $g$ so that $c_{g}$ in (3.12) belongs to $C^{0,1}(\mathbb{R})$ and for any initial data $\mathbf{w}^{h, 0}$ in 3.10 bounded in $\ell^{2}$ as $h \rightarrow 0$, there exists a positive Radon measure $\mathcal{W}=\mathcal{W}(x, t, \xi)$ defined on $\mathbb{R} \times \mathbb{R}_{+} \times[-\pi, \pi]$ such that, eventually after extracting a subsequence, we get

$$
\mathcal{W}^{h}\left[\mathbf{w}^{h}\right](x, t, \xi) \rightarrow \mathcal{W}(x, t, \xi) \text { weakly star in } \mathcal{S}^{\prime}\left(\mathbb{R}_{x}\right) \times \mathcal{D}^{\prime}([-\pi, \pi]),
$$

uniformly on each compact set of the time interval $\mathbb{R}_{+}$. Moreover, the measure $\mathcal{W}$ satisfies the Liouville equation (we follow the terminology in [21])

$$
\partial_{t} \mathcal{W}(x, t, \xi)=\mp \mathrm{P}_{c_{g}, \omega} \mathcal{W}(x, t, \xi), \mathcal{W}(x, 0, \xi)=\mathcal{W}^{0}(x, \xi):=\lim _{h \rightarrow 0} \mathcal{W}^{h}\left[w^{h, 0}\right](x, \xi)
$$

with $\omega(\xi)=\sin (\xi)$ and the operator $\mathrm{P}_{c, \omega}$ defined as in 2.13).

Remark 1. Theorem 3.3 states that the Wigner measure corresponding to the discrete transport equation (3.10) propagates according to a transport equation in both phase-space variables $(x, \xi)$ whose solution propagates along the characteristic rays described by the Hamiltonian system (3.8).

Remark 2. A particular class of bounded initial data in $\ell^{2}$ as $h \rightarrow 0$ is given by $\mathbf{w}^{h, 0}:=\left(u^{h, 0}\left(x_{j}\right)\right)_{j \in \mathbb{Z}}$, with $u^{h, 0}$ as in 2.21.

Remark 3. The dynamics of the solution to the discrete problem (3.10) is determined by the symbol $\lambda_{\gamma^{h}, \omega}(x, \xi) \sim \lambda_{c_{g}, \omega}(x, \xi)=c_{g}(x) \omega(\xi)(\omega(\xi)=\sin (\xi))$ generating the leading pseudo-differential operator in (3.14) (i.e. the term of order $h^{-1}$ in the right hand side of (3.14)).

3.3. The discrete wave equation. The following convergence result for the solution of the discrete wave equation (1.7) holds (the corresponding proof is provided in Appendix A):

Proposition 3.4. Assume $\rho \in C^{1}(\mathbb{R})$ and $\sigma \in C^{2}(\mathbb{R})$ in 1.4) such that there exist $\rho^{-}, \rho^{+}, \rho_{d}^{+}$and $\sigma^{-}, \sigma^{+}, \sigma_{d d}^{+}$so that $0<\rho^{-} \leq \rho(y) \leq \rho^{+},\left|\rho^{\prime}(y)\right| \leq \rho_{d}^{+}, 0<\sigma^{-} \leq \sigma(y) \leq \sigma^{+},\left|\sigma^{\prime \prime}(y)\right| \leq \sigma_{d d}^{+}$, for all $y \in \mathbb{R}$, and compactly supported initial data $\left(u^{0}, u^{1}\right) \in C^{3} \times C^{2}(\mathbb{R})$. We also consider non-uniform grids $\mathcal{G}_{g}^{h}$ given by $g \in C^{2}(\mathbb{R})$ such that there exists $g_{d}^{+}, g_{d}^{-}, g_{d d}^{+}>0$ so that $0<g_{d}^{-} \leq\left|g^{\prime}(x)\right| \leq g_{d}^{+}$and $\left|g^{\prime \prime}(x)\right| \leq g_{d d}^{+}$, for all $x \in \mathbb{R}$. Under these hypotheses, the numerical approximation (1.6) with initial data $\left(\mathbf{u}^{h, 0}, \mathbf{u}^{h, 1}\right):=\left(u^{0}\left(g_{j}\right), u^{1}\left(g_{j}\right)\right)_{j \in \mathbb{Z}}$ is a convergent scheme of order $O(h)$ for the wave equation (1.4) in the $\dot{\hbar}^{1} \times \ell^{2}$-norm, i.e. there exists a constant $C\left(t, g, \rho, \sigma, u^{0}, u^{1}\right)$ independent of $h$ such that

$$
\left\|\left(\mathbf{u}^{h}(t)-u\left(g\left(\mathbf{x}^{h}\right), t\right), \partial_{t} \mathbf{u}^{h}(t)-\partial_{t} u\left(g\left(\mathbf{x}^{h}\right), t\right)\right)\right\|_{\hbar^{1} \times \ell^{2}} \leq h C\left(t, g, \rho, \sigma, u^{0}, u^{1}\right) .
$$

The function $v(x, t):=u(g(x), t)(u(y, t)$ being the solution of 1.4$)$ solves the wave equation

$$
g^{\prime}(x) \rho(g(x)) \partial_{t}^{2} v-\partial_{x}\left(\frac{\sigma(g(x))}{g^{\prime}(x)} \partial_{x} v\right)=0, v(x, 0)=u^{0}(g(x)), \partial_{t} v(x, 0)=u^{1}(g(x)), x \in \mathbb{R}, t>0 .
$$

Thus, by reconsidering the values $u_{j}(t)$ associated initially to the points of the non-uniform grid as being values $v_{j}(t)=u_{j}(t)$ at points of the uniform grid, we obtain that $v_{j}(t)$ is the solution of the finite difference approximation for the wave equation (3.18) on the uniform grid $\mathcal{G}^{h}$

$$
\partial^{h} g_{j} \rho\left(g_{j}\right) \partial_{t}^{2} v_{j}(t)-\frac{\frac{\sigma\left(g_{j+1 / 2}\right)}{\partial^{h,+} g_{j}} \partial^{h,+} v_{j}(t)-\frac{\sigma\left(g_{j-1 / 2}\right)}{\partial^{h,-} g_{j}} \partial^{h,-} v_{j}(t)}{h}=0 .
$$

Indeed, from Proposition 3.4, we see that $\mathbf{u}^{h}(t)=\mathbf{v}^{h}(t) \sim v\left(\mathbf{x}^{h}, t\right)=u(g(x), t)$, where $\left(v(x, t), \mathbf{v}^{h}(t)\right)$ are the solutions of $([3.18),(3.19))$. 
The null bi-characteristic lines associated to the principal symbol 1.100 of the numerical approximation (3.19) of the wave equation are the solutions of the following system:

$$
\left\{\begin{array}{l}
X^{\prime}(s)=\partial_{\xi} \wp=2 \sin (\Xi(s)) \frac{\sigma(g(X(s)))}{g^{\prime}(X(s))}, t^{\prime}(s)=\partial_{\tau} \wp=-2 g^{\prime}(X(s)) \rho(g(X(s))) \tau, \\
\Xi^{\prime}(s)=-\partial_{x} \wp=\tau^{2}\left(g^{\prime}(\cdot) \rho(g(\cdot))\right)^{\prime}(X(s))-4 \sin ^{2}\left(\frac{\Xi(s)}{2}\right)\left(\frac{\sigma(g(\cdot))}{g^{\prime}(\cdot)}\right)^{\prime}(X(s)), \tau^{\prime}(s)=-\partial_{t} \wp=0,
\end{array}\right.
$$

subjected to the initial data $(X(0), t(0), \Xi(0), \tau(0))=\left(x_{0}, 0, \xi_{0}, \tau_{0}\right)$ such that $\wp\left(x_{0}, 0, \xi_{0}, \tau_{0}\right)=0$. Then $\tau(s)=\tau_{0}$ and $\wp(X(s), t(s), \Xi(s), \tau(s))=0$ are conserved in $s$. Set $\left(x^{ \pm}(t), \xi^{ \pm}(t)\right):=(X(s), \Xi(s))$ to be the solution of (3.20) corresponding to one of the two possible roots $\tau_{0}^{ \pm}$of $\wp\left(X(s), t(s), \Xi(s), \tau_{0}\right)=0$ given by $\tau_{0}^{ \pm}= \pm 2 \sin (\Xi(s) / 2) c_{g}(X(s))$, with $c_{g}(x):=\sqrt{\sigma(g(x)) / \rho(g(x))} / g^{\prime}(x)$. Then $\left(x^{ \pm}(t), \xi^{ \pm}(t)\right)$ is the solution of the following Hamiltonian system:

$$
\left(x^{ \pm}\right)^{\prime}(t)=\mp c_{g}\left(x^{ \pm}(t)\right) \cos \left(\frac{\xi^{ \pm}(t)}{2}\right),\left(\xi^{ \pm}\right)^{\prime}(t)= \pm 2 c_{g}^{\prime}\left(x^{ \pm}(t)\right) \sin \left(\frac{\xi^{ \pm}(t)}{2}\right), x(0)=x_{0}, \quad \xi(0)=\xi_{0} .
$$

Remark 4. The Hamiltonian system (3.21) can be also obtained by considering in (3.20) the principal symbol $\wp(x, t, \xi, \tau)$ below instead of 1.10$)$

$$
\wp(x, t, \xi, \tau):=-\rho(g(x)) \tau^{2}+4 \sin ^{2}\left(\frac{\xi}{2}\right) \frac{\sigma(g(x))}{\left(g^{\prime}(x)\right)^{2}} .
$$

This last symbol can be seen intuitively by taking plane wave solutions of the form $u_{j}(t)=\exp (i \tau t / h+i \xi j)$ in the numerical scheme (1.7) on the non-uniform grid $\mathcal{G}_{g}^{h}$.

Let us consider the following continuous functions:

$$
\begin{gathered}
\alpha^{h}(x):=\frac{1}{\sqrt{\partial^{h} g(x) \rho(g(x))}}, \quad \beta^{h}(x):=\sqrt{\frac{\sigma(g(x+h / 2))}{\partial^{h,+} g(x)}}, \\
\gamma^{h}(x):=\alpha^{h}(x) \beta^{h}(x) \text { and } \delta^{h, \pm}(x):=\beta^{h}(x) \partial^{h,+} \alpha^{h}(x) \pm \alpha^{h}(x) \partial^{h,-} \beta^{h}(x) .
\end{gathered}
$$

Associated to them, we consider the discrete functions $\alpha_{j}:=\alpha^{h}\left(x_{j}\right), \beta_{j}:=\beta^{h}\left(x_{j}\right), \gamma_{j}:=\gamma^{h}\left(x_{j}\right)$ and $\delta_{j}^{ \pm}:=\delta^{h, \pm}\left(x_{j}\right)$, for all $j \in \mathbb{Z}$. Remark that, as $h \rightarrow 0$, the following approximations hold:

$$
\alpha^{h}(x) \sim \frac{1}{\sqrt{\rho(g(x)) g^{\prime}(x)}}, \quad \beta^{h}(x) \sim \sqrt{\frac{\sigma(g(x))}{g^{\prime}(x)}}, \quad \gamma^{h}(x) \sim c_{g}(x):=\frac{1}{g^{\prime}(x)} \sqrt{\frac{\sigma(g(x))}{\rho(g(x))}}
$$

and

$$
\delta^{h,+}(x) \sim c_{g}^{\prime}(x) \text { and } \delta^{h,-}(x) \sim \frac{\sigma(g(x))}{g^{\prime}(x)}\left(\frac{1}{\sqrt{\rho(g) \sigma(g)}}\right)^{\prime}(x) .
$$

In the semi-discrete wave equation 3.19 , set $w_{j}(t):=v_{j}^{\prime}(t) / \alpha_{j}$ and $\tilde{w}_{j}(t):=\beta_{j} \partial^{h,+} v_{j}(t)$. Due to the conservation of energy, the norm $\left\|\left(\mathbf{w}^{h}(t), \tilde{\mathbf{w}}^{h}(t)\right)\right\|_{\left(\ell^{2}\right)^{2}}$ is conserved in time. Furthermore, the two functions $\mathbf{w}^{h}(t)$ and $\tilde{\mathbf{w}}^{h}(t)$ are the solutions of the coupled system of discrete transport equations

$$
\partial_{t} w_{j}(t)=\gamma_{j} \partial^{h,-} \tilde{w}_{j}(t)+\alpha_{j}\left(\partial^{h,-} \beta_{j}\right) \tilde{w}_{j-1}(t), \quad \partial_{t} \tilde{w}_{j}(t)=\gamma_{j} \partial^{h,+} w_{j}(t)+\beta_{j}\left(\partial^{h,+} \alpha_{j}\right) w_{j+1},
$$

with initial data $w_{j}(0)=w_{j}^{0}:=u_{j}^{1} / \alpha_{j}$ and $\tilde{w}_{j}(0)=\tilde{w}_{j}^{0}:=\beta_{j} \partial^{h,+} u_{j}^{0}$, for all $j \in \mathbb{Z}$.

Let us observe that (3.24) can be also written in terms of the two pseudo-differential operators $\mathbf{\Theta}_{1}^{h}\left(x, h \partial_{x}\right)$ and $\boldsymbol{\Theta}_{0}^{h}\left(x, h \partial_{x}\right)$ (here, the subscripts 0 and 1 indicate the smallest power of $\xi$ appearing in the Taylor expansions around $\xi=0$ of $\boldsymbol{\Theta}_{1}$ and $\boldsymbol{\Theta}_{0}$ for a fixed $x$ ) generated by the matrices

$$
\boldsymbol{\Theta}_{1}(x, \xi):=\left(\begin{array}{cc}
0 & \gamma^{h}(x)(1-\exp (-i \xi)) \\
\gamma^{h}(x)(\exp (i \xi)-1) & 0
\end{array}\right)
$$

and

as follows

$$
\boldsymbol{\Theta}_{0}(x, \xi):=\left(\begin{array}{cc}
0 & \alpha^{h}(x) \partial^{h,-} \beta^{h}(x) \exp (-i \xi) \\
\beta^{h}(x) \partial^{h,+} \alpha^{h}(x) \exp (i \xi) & 0
\end{array}\right)
$$

$$
\left(\begin{array}{c}
\partial_{t} \mathbf{w}^{h}(t) \\
\partial_{t} \tilde{\mathbf{w}}^{h}(t)
\end{array}\right)=\frac{1}{h} \Theta_{1}^{h}\left(x, h \partial_{x}\right)\left(\begin{array}{c}
\mathbf{w}^{h}(t) \\
\tilde{\mathbf{w}}^{h}(t)
\end{array}\right)+\Theta_{0}^{h}\left(x, h \partial_{x}\right)\left(\begin{array}{c}
\mathbf{w}^{h}(t) \\
\tilde{\mathbf{w}}^{h}(t)
\end{array}\right)
$$


The matrix $\boldsymbol{\Theta}_{1}(x, \xi)$ admits the spectral decomposition $\boldsymbol{\Theta}_{1}(x, \xi)=i \boldsymbol{\Delta}(\xi) \boldsymbol{\Lambda}(x, \xi) \boldsymbol{\Delta}^{*}(\xi)$ ( $\boldsymbol{A}^{*}$ being the conjugate transpose of the matrix $\boldsymbol{A}$ ), where

$$
\boldsymbol{\Lambda}(x, \xi):=\left(\begin{array}{cc}
\lambda_{\gamma^{h}, \omega}^{+}(x, \xi) & 0 \\
0 & \lambda_{\gamma^{h}, \omega}^{-}(x, \xi)
\end{array}\right), \quad \boldsymbol{\Delta}(\xi):=\frac{1}{\sqrt{2}}\left(\begin{array}{cc}
1 & 1 \\
\exp (i \xi / 2) & -\exp (i \xi / 2)
\end{array}\right),
$$

with $\lambda_{\gamma^{h}, \omega}^{ \pm}(x, \xi)= \pm \gamma^{h}(x) \omega(\xi), \gamma^{h}$ as in 3.22 and $\omega(\xi)=2 \sin (\xi / 2)$. Note that the eigenvalues $\lambda_{\gamma^{h}, \omega}^{ \pm}$of $\boldsymbol{\Theta}_{1}$ have the same structure in separated variable of the symbol $\lambda_{\gamma^{h}, \omega}(x, \xi)=\gamma^{h}(x) \omega(\xi)$ generating the leading pseudo-differential operator in the transport equation (3.14). Thus, $\gamma^{h}(x)$ is given approximately by a function $c_{g}(x)$ for both models, where the functions $c_{g}$ are given by $(3.12)$ and $(3.23)$ for the discrete transport/wave equations and depend on the variable coefficients appearing in the corresponding continuous model and on the grid mapping $g$. Another difference between $\lambda_{\gamma^{h}, \omega}(x, \xi)$ and $\lambda_{\gamma^{h}, \omega}^{ \pm}(x, \xi)$ in $(3.14)$ and $(3.25)$ is the Fourier symbol $\omega$, so that $\omega(\xi)=\sin (\xi)$ and $\omega(\xi)=2 \sin (\xi / 2)$ in the first/second case. These trigonometric functions $\omega$ are simply the classical dispersion relations for the finite difference semi-discretizations of the constant coefficients transport/wave equations on uniform meshes.

Remark also that the matrix of eigenvectors $\boldsymbol{\Delta}(\xi)$ in 3.26 does not depend on the space variable $x$. Let us introduce the projectors for the numerical scheme (3.24) to be

$$
\Delta^{+}:=\Delta\left(\begin{array}{ll}
1 & 0 \\
0 & 0
\end{array}\right) \Delta^{*} \text { and } \Delta^{-}:=\Delta\left(\begin{array}{ll}
0 & 0 \\
0 & 1
\end{array}\right) \Delta^{*}, \text { i.e. } \Delta^{ \pm}=\frac{1}{2}\left(\begin{array}{cc}
1 & \pm \exp (-i \xi / 2) \\
\pm \exp (i \xi / 2) & 1
\end{array}\right)
$$

where $\Delta$ is the matrix containing the eigenvectors of $\Theta_{1}$ in $(3.26)$. Let us denote the solution of (3.25) by $\mathbf{w}^{\mathbf{h}}(t)=\left(\mathbf{w}^{h}(t), \tilde{\mathbf{w}}^{h}(t)\right)$ and by $\mathcal{W}^{h}\left[\mathbf{w}^{\mathbf{h}}\right]\left(x_{m} / 2, t, \xi\right)$ the Wigner transform matrix of $\mathbf{w}^{\mathbf{h}}$. Set

$$
\mathcal{W}^{h, \pm}\left(\frac{x_{m}}{2}, t, \xi\right):=\Delta^{ \pm}(\xi) \mathcal{W}^{h}\left[\mathbf{w}^{\mathbf{h}}\right]\left(\frac{x_{m}}{2}, t, \xi\right) \Delta^{ \pm}(\xi) \text { and } \mathcal{W}^{h, \pm}\left(\frac{x_{m}}{2}, t, \xi\right):=\operatorname{tr}\left(\mathcal{W}^{h, \pm}\left(\frac{x_{m}}{2}, t, \xi\right)\right)
$$

Here, $\operatorname{tr}(\mathbf{A})$ is the trace of the matrix A. Our second result (also to be proved in Section 4 states that the Wigner measure of $\mathbf{w}^{\mathbf{h}}(t)$ can be decomposed into two parts following two Wigner measures as $h \rightarrow 0$, each of them satisfying a transport equation in the space-phase variables $(x, \xi)$. Firstly, set $\mathcal{W}^{h}\left(x_{m} / 2, t, \xi\right):=$ $\operatorname{tr}\left(\mathcal{W}^{h}\left[\mathbf{w}^{\mathbf{h}}\right]\left(x_{m} / 2, t, \xi\right)\right)$.

Theorem 3.5. For each coefficient $\rho$ and $\sigma$ in 1 (1.4), each non-uniform grid $\mathcal{G}_{g}^{h}$ obtained by transformations $g$ so that $c_{g}$ in (3.23) belongs to $C^{1,1}(\mathbb{R})$ and for any initial data $\left(\mathbf{w}^{h, 0}, \tilde{\mathbf{w}}^{h, 0}\right)$ in (3.24) bounded in $\ell^{2}$ as $h \rightarrow 0$, there exists a positive Radon measure $\mathcal{W}=\mathcal{W}(x, t, \xi)$ defined on $\mathbb{R} \times \mathbb{R}_{+} \times[-\pi, \pi]$ such that, eventually after extracting subsequences, we get (3.15) uniformly on each compact set of the time interval $\mathbb{R}_{+}$. Moreover, the measure $\mathcal{W}$ can be decomposed as $\mathcal{W}=\mathcal{W}^{+}+\mathcal{W}^{-}$, where $\mathcal{W}^{ \pm}=\lim _{h \rightarrow 0} \mathcal{W}^{h, \pm}\left(\mathcal{W}^{h, \pm}\right.$ as in (3.28)) satisfies the Liouville equation

$$
\partial_{t} \mathcal{W}^{ \pm}(x, t, \xi)= \pm \mathcal{P}_{c_{g}}(x, \omega) \mathcal{W}^{ \pm}(x, t, \xi), \mathcal{W}^{ \pm}(x, 0, \xi)=\mathcal{W}^{ \pm, 0}(x, \xi):=\lim _{h \rightarrow 0} \mathcal{W}^{h, \pm}(x, 0, \xi) .
$$

Here, the operator $\mathcal{P}_{c_{g}}(x, \omega(\xi))$ is defined by 2.13) with $c=c_{g}$ given by 3.23) and $\omega(\xi)=2 \sin (\xi / 2)$.

Remark 5. The propagation properties of the discrete wave equation (3.24) are determined by the two eigenvalues $\lambda_{\gamma^{h}, \omega}^{ \pm}(x, \xi) \sim \lambda_{c_{g}, \omega}^{ \pm}(x, \xi)$ of the principal symbol $\boldsymbol{\Theta}_{1}(x, \xi)$ in 3.25).

The following consequence of Theorem 3.5 (whose proof relies on the same arguments presented in Subsection ?? and based on the discrete Wigner transform and its properties) holds:

Corollary 3.6. The energy of the solution $\mathbf{w}^{\mathbf{h}}(t)=\left(\mathbf{w}^{h}(t), \tilde{\mathbf{w}}^{h}(t)\right)$ of (3.24) can be arbitrarily concentrated along one of the rays $x^{ \pm}(t)$ in (3.21), in the sense that for any $r>0$ and for the initial data in (3.24)

$$
\mathbf{w}^{h, 0}=\left( \pm \alpha_{j}^{-1} c_{g}\left(x_{j}\right) \partial^{h,+} u^{h, 0}\left(x_{j}\right)\right)_{j \in \mathbb{Z}} \text { and } \tilde{\mathbf{w}}^{h, 0}=\left(\beta_{j} \partial^{h,+} u^{h, 0}\left(x_{j}\right)\right)_{j \in \mathbb{Z}},
$$

we get

$$
\lim _{h \rightarrow 0} h \sum_{\left|x_{j}-x^{ \pm}(t)\right|>r}\left(\left|w_{j}(t)\right|^{2}+\left|\tilde{w}_{j}(t)\right|^{2}\right)=0
$$


Here, $x^{ \pm}(t)$ is the solution of (3.21) with data $\left(x_{0}, \xi_{0}\right), \alpha_{j}, \beta_{j}$ are as in (3.22), $u^{\epsilon, 0}$ is as in (2.22) with $y_{0}$ replaced by $x_{0}:=g^{-1}\left(y_{0}\right)$ and $f$ is such that (with $\omega(\xi):=2 \sin (\xi / 2)$ )

$$
\sigma\left(y_{0}\right)\left|\omega\left(\xi_{0}\right)\right|^{2}\|f\|_{L^{2}(\mathbb{R})}^{2} / g^{\prime}\left(g^{-1}\left(y_{0}\right)\right)=1 .
$$

Of course, this Corollary 3.6 and the fact that there exists a constant $g_{d}^{-}>0$ such that $\left|g^{\prime}(x)\right| \geq g_{d}^{-}$ for all $x \in \mathbb{R}$ imply that the energy of the original discrete wave equation (1.7) on the non-uniform grid $\mathcal{G}_{g}^{h}$ corresponding to the initial data $\mathbf{u}^{h, 0}=\left(u^{h, 0}\left(g^{-1}\left(g_{j}\right)\right)\right)_{j \in \mathbb{Z}}$ and $\mathbf{u}^{h, 1}=\left( \pm c_{g}\left(x_{j}\right) \partial^{h,+} u_{j}^{0}\right)_{j \in \mathbb{Z}}$ may be concentrated arbitrarily close to the ray $y^{ \pm}(t):=g\left(x^{ \pm}(t)\right)$.

3.4. Group velocity. We define the group velocity associated to the numerical schemes (1.6) and (1.7) for the transport or the wave equations to be $\left(x^{\mp}\right)^{\prime}(t)$ (i.e. $\mp c_{g}\left(x^{\mp}(t)\right) \cos \left(\xi^{\mp}(t)\right.$ ), where $c_{g}$ is as in 3.12 ) and $\left(x^{\mp}(t), \xi^{\mp}(t)\right)$ is the solution of $(3.8)$ ) or $\left(x^{ \pm}\right)^{\prime}(t)$ (i.e. $\mp c_{g}\left(x^{ \pm}(t)\right) \cos \left(\xi^{ \pm}(t) / 2\right)$, where $c_{g}$ is as in (3.23) and $\left(x^{ \pm}(t), \xi^{ \pm}(t)\right)$ is the solution of (3.21) ) (cf. [3], pp. 65).

This definition of the group velocity is consistent with the well-known one for the homogeneous discrete medium (i.e. constant coefficients and on a uniform mesh) (see [1], 33] or [35). In that case, $c_{g} \equiv 1$, so that the solution of the second equations in (3.8) and (3.21) is $\xi^{ \pm}(t)= \pm \xi_{0}$. Consequently, the first equations in (3.8) and (3.21) become $\left(x^{\mp}\right)^{\prime}(t)=\mp \cos \left(\xi_{0}\right)$ and $\left(x^{ \pm}\right)^{\prime}(t)=\mp \cos \left(\xi_{0} / 2\right)$, so that the group velocity does not depend on time and the characteristics $x^{ \pm}(t)$ are simply straight lines.

It is well-known that, for homogeneous media, a vanishing group velocity (at $\xi_{0}=\pi / 2$ or at $\xi_{0}=\pi$ for the transport/wave equation) yields spurious solutions that do not propagate in time. These fictitious solutions make the uniform (with respect to the mesh size) observability property to fail. In that case, it is necessary to apply filtering techniques on the initial data (e.g. Fourier truncation, bi-grid algorithms) to avoid the high frequency pathological effects (cf. [11, 35]).

However, for heterogeneous media (variable coefficients and/or non-uniform meshes), the group velocity depends on the time $t$ through the two components $(x(t), \xi(t))$ or $\left(x^{ \pm}(t), \xi^{ \pm}(t)\right)$ solving (3.8) or (3.21).

3.5. Related bibliography. In 2] and 32 the problem of computing spectral distributions for locally Toeplitz matrix sequences has been considered and the results have been applied to design efficient preconditioning strategies for linear systems arising in the numerical approximation of elliptic PDEs in [30.

Locally Toeplitz matrices appear naturally, indeed, in the discretization process of PDEs with variable coefficients on non-uniform meshes $\mathcal{G}_{g}^{h}, g:[0,1] \rightarrow[0,1], g \in C^{1}(0,1)$. For simplicity, consider the $1-d$ Laplace problem

$$
-\left(\sigma(y) u^{\prime}\right)^{\prime}(y)=f(y), \quad y \in(0,1), \quad u(0)=u(1),
$$

for which a typical discretization is the three-points finite difference scheme below $(h:=1 /(N+1), N \in \mathbb{N})$

$$
\mathbf{A}_{g}^{h} \mathbf{u}^{h}=\mathbf{f}^{h} \text { or, more precisely, }-\frac{1}{\partial^{h} g_{j}} \partial^{h,-}\left(\frac{\sigma_{j+1 / 2}}{\partial^{h,+} g_{j}} \partial^{h,+} u_{j}\right)=f_{j}, \quad 1 \leq j \leq N, \quad u_{0}=u_{N+1}=0 .
$$

When $\sigma$ is a continuous function, any sub-block of fixed size of the matrix of the system $\mathbf{A}_{g}^{h}$ tends to a Toeplitz matrix as $N \rightarrow \infty$. The spectral distribution $\mu$ of the sequence of stiffness matrices of this scheme is the measure defined as $\langle\mu, F\rangle=\lim _{N \rightarrow \infty} N^{-1} \sum_{j=1}^{N} F\left(\lambda_{j}\right)$, where $\left(\lambda_{j}\right)_{1 \leq j \leq N}$ are the eigenvalues of $\mathbf{A}_{g}^{h}$ and $F$ is any $C_{c}(\mathbb{R})$-function. In [2] (variable coefficients and non-uniform meshes) and [32] (variable coefficients and uniform meshes), it is proved that the spectral distribution satisfies the identity $\langle\mu, F\rangle=$ $\int_{0}^{1} \int_{-\pi}^{\pi} F\left(\lambda^{2}(x, \xi)\right) d \xi d x / 2 \pi$, where $\lambda^{2}(x, \xi):=\sigma(g(x)) \omega^{2}(\xi) /\left|g^{\prime}(x)\right|^{2}$ and $\omega^{2}(\xi)=4 \sin ^{2}(\xi / 2)$ is the Fourier symbol of the three-point scheme for the Laplace equation with constant coefficients on the uniform mesh $\mathcal{G}^{h}$. This is in agreement with our results for the wave equation (1.4), for which the characteristics depend on the symbol $\lambda(x, \xi):=c(g(x)) \omega(\xi) / g^{\prime}(x)$, with $c:=\sqrt{\sigma / \rho}$.

In [9], the observability problem for mixed finite element semi-discretizations of the $1-d$ wave equation has been considered. More precisely, for quasi-uniform meshes (for which the ratio between the maximum cell size and the minimum one remains bounded under all refinements of the mesh), it is proved that the approximation under consideration inherits its well-known good features on uniform grids, for which observability holds uniformly with respect to the mesh-size parameter. However, the results are based on 
a precise description of the spectrum of the numerical scheme on non-uniform meshes and they cannot be generalized to other numerical approximations.

In [10, it is shown via spectral estimates that for all convergent numerical approximations (thus, including the ones on non-uniform meshes), after truncating some high frequency modes, one can always prove uniform observability estimates for the remaining low frequency ones. Our analysis in this paper shows the necessity of such filtering mechanisms for finite difference numerical schemes on non-uniform meshes for the $1-d$ wave equation.

In [23, Macià analyzes to which extent the oscillation and the concentration effects of a sequence of functions are preserved or eliminated by sampling and reconstruction operators on uniform meshes. The main tool of that analysis is based, as in this paper, on Wigner measures. The oscillation/concentration effects of samplings of continuous functions analyzed in that paper are important ingredients when dealing with the Wigner measure of the initial data in the discrete problems $(3.10)$ and (3.24). Thus, our analysis on the evolution problems under consideration combined with the one in [23] on the initial data give the complete picture on the behavior of the Wigner measures leaded by the Cauchy problems (3.16) and (3.29).

\section{Proof of the main Results}

4.1. Proof of Theorem $\mathbf{3 . 3}$ concerning the discrete transport equation $(\mathbf{1 . 6})$. We follow the same steps in the proof of Theorem 2.1 in Section 2. For the simplicity of notation, in this proof we write $\mathcal{W}^{h}$ instead of $\mathcal{W}^{h}\left[\mathbf{w}^{h}\right]$.

Step I. Using the boundedness as $h \rightarrow 0$ of the $\ell^{2}$-norm of the initial data $\mathbf{w}^{h, 0}$ in (3.10) and the time conservation of the $\ell^{2}$-norm of $\mathbf{w}^{h}(\cdot, t)$, we obtain existence of a positive Radon measure $\mathcal{W}$ such that

$$
\mathcal{W}^{h}(x, t, \xi) \rightarrow \mathcal{W}(x, t, \xi) \text { weakly star in } \mathcal{S}^{\prime}\left(\mathbb{R}_{x}\right) \times \mathcal{D}^{\prime}([-\pi, \pi]) \text { as } h \rightarrow 0, \forall t \geq 0,
$$

Step II. Set $\widehat{\mathcal{W}}^{h}\left(x_{m} / 2, t, n\right)$ to be the $n$-th Fourier coefficient of $\mathcal{W}^{h}\left(x_{m} / 2, t, \xi\right)$ as function of $\xi$. Following (3.3), it is easy to see that

$$
\widehat{\mathcal{W}}^{h}\left(\frac{x_{m}}{2}, t, n\right)=w_{\frac{m-n}{2}}(t) \bar{w}_{\frac{m+n}{2}}(t) .
$$

Define $\partial_{x}^{h}$ and $\mathfrak{M}_{x}^{h}$ to be the centered derivative/average operators in the variable $x$. More precisely, $\partial_{x}^{h} f\left(x_{m} / 2, \varsigma\right):=\left(f\left(x_{m+1} / 2, \varsigma\right)-f\left(x_{m-1} / 2, \varsigma\right)\right) / h$ and $\mathfrak{M}_{x}^{h} f\left(x_{m} / 2, \varsigma\right):=\left(f\left(x_{m+1} / 2, \varsigma\right)+f\left(x_{m-1} / 2, \varsigma\right)\right) / 2$, where $\varsigma$ stores the other possible variables defining the function $f(x, \varsigma)$. By taking time derivative in 4.2 . and using the numerical scheme 3.10 , we get the following equation verified by $\widehat{\mathcal{W}}^{h}\left(x_{m} / 2, t, n\right)$ :

$$
\begin{aligned}
\partial_{t} \widehat{\mathcal{W}}^{h}\left(\frac{x_{m}}{2}, t, n\right) & =-\left(\frac{1}{2} \mathcal{K}_{\gamma^{h}}^{h,+}\left(\frac{x_{m}}{2}, n\right)\left(\partial_{x}^{h} \widehat{\mathcal{W}}^{h}\left(\frac{x_{m}}{2}, t, n+1\right)+\partial_{x}^{h} \widehat{\mathcal{W}}^{h}\left(\frac{x_{m}}{2}, t, n-1\right)\right)\right. \\
& +\frac{1}{2} \mathcal{K}_{\gamma^{h}}^{h,-}\left(\frac{x_{m}}{2}, n\right)\left(\mathfrak{M}_{x}^{h} \widehat{\mathcal{W}}^{h}\left(\frac{x_{m}}{2}, t, n+1\right)-\mathfrak{M}_{x}^{h} \widehat{\mathcal{W}}^{h}\left(\frac{x_{m}}{2}, t, n-1\right)\right) \\
& +\mathcal{K}_{\delta^{h}}^{h,+}\left(\frac{x_{m}}{2}, n\right)\left(\mathfrak{M}_{x}^{h} \widehat{\mathcal{W}}^{h}\left(\frac{x_{m}}{2}, t, n+1\right)+\mathfrak{M}_{x}^{h} \widehat{\mathcal{W}}^{h}\left(\frac{x_{m}}{2}, t, n-1\right)\right) \\
& +\frac{h^{2}}{4} \mathcal{K}_{\delta^{h}}^{h,-}\left(\frac{x_{m}}{2}, n\right)\left(\partial_{x}^{h} \widehat{\mathcal{W}}^{h}\left(\frac{x_{m}}{2}, t, n+1\right)-\partial_{x}^{h} \widehat{\mathcal{W}}^{h}\left(\frac{x_{m}}{2}, t, n-1\right)\right),
\end{aligned}
$$

where $\mathcal{K}_{c}^{h, \pm}$ are as in A.2 (with $\epsilon=h$ ) and $\gamma^{h}, \delta^{h}$ are as in (3.11).

Step III. Set

$$
\left\langle\widehat{f}_{1}, \widehat{f}_{2}\right\rangle_{h}:=\frac{1}{2 \pi} \frac{h}{2} \sum_{m \in \mathbb{Z}} \sum_{n \equiv m} \widehat{f}_{1}\left(\frac{x_{m}}{2}, n\right) \widehat{f}_{2}\left(\frac{x_{m}}{2}, n\right) \text { and }\left\langle f_{1}, f_{2}\right\rangle_{h, \mathcal{D}^{\prime}, \mathcal{D}}:=\frac{h}{2} \sum_{m \in \mathbb{Z}} \int_{-\pi}^{\pi} f_{1}\left(\frac{x_{m}}{2}, \xi\right) f_{2}\left(\frac{x_{m}}{2}, \xi\right) d \xi \text {. }
$$

Consider $a \in \mathcal{S}_{x}(\mathbb{R}) \times \mathcal{D}_{\xi}([-\pi, \pi])$. Let us multiply 4.3 by $h \overline{\bar{a}}\left(x_{m} / 2, n\right)$ and add in $m, n \in \mathbb{Z}$ so that $n \equiv m \bmod 2$. By Parseval identity in $\xi$, we obtain:

$$
\left\langle\partial_{t} \mathcal{W}^{h}(\cdot, t, \cdot), a\right\rangle_{h, \mathcal{D}^{\prime}, \mathcal{D}}=\left\langle\partial_{t} \widehat{\mathcal{W}}^{h}(\cdot, t, \cdot), \overline{\bar{a}}\right\rangle_{h}=-\left(\mathcal{I}_{1}^{h}(t)+\mathcal{I}_{2}^{h}(t)+\mathcal{I}_{3}^{h}(t)+\mathcal{I}_{4}^{h}(t)\right),
$$


where

$$
\begin{aligned}
& \mathcal{I}_{1}^{h}(t)=\left\langle\partial_{x}^{h} \widehat{\mathcal{W}}^{h}(\cdot, t, \cdot+1)+\partial_{x}^{h} \widehat{\mathcal{W}}^{h}(\cdot, t, \cdot-1), \frac{1}{2} \mathcal{K}_{\gamma^{h}}^{h+}+\overline{\bar{a}}\right\rangle_{h}, \mathcal{I}_{2}^{h}(t)=\left\langle\mathfrak{M}_{x}^{h} \widehat{\mathcal{W}}^{h}(\cdot, t, \cdot+1)-\mathfrak{M}_{x}^{h} \widehat{\mathcal{W}}^{h}(\cdot, t, \cdot-1), \frac{1}{2} \mathcal{K}_{\gamma^{h}}^{h,-\overline{\bar{a}}\rangle_{h},}\right. \\
& \mathcal{I}_{3}^{h}(t)=\left\langle\mathfrak{M}_{x}^{h} \widehat{\mathcal{W}}^{h}(\cdot, t, \cdot+1)+\mathfrak{M}_{x}^{h} \widehat{\mathcal{W}}^{h}(\cdot, t, \cdot-1), \mathcal{K}_{\delta^{h}}^{h,+} \overline{\bar{a}}\right\rangle_{h}, \mathcal{I}_{4}^{h}(t)=\left\langle\partial_{x}^{h} \widehat{\mathcal{W}}^{h}(\cdot, t, \cdot+1)-\partial_{x}^{h} \widehat{\mathcal{W}}^{h}(\cdot, t, \cdot-1), \frac{h^{2}}{4} \mathcal{K}_{\delta^{h}}^{h,-} \overline{\bar{a}}\right\rangle_{h} .
\end{aligned}
$$

By passing all the discrete derivatives and averages from $\widehat{\mathcal{W}}^{h}$ to the accompanying factors and applying Parseval identity, we get

$$
\mathcal{I}_{j}^{h}(t):=\left\langle\mathcal{W}^{h}(\cdot, t, \cdot),\left(\overline{a \sharp c_{g}}\right)_{j}^{h}\right\rangle_{h, \mathcal{D}^{\prime}, \mathcal{D}},
$$

where

$$
\begin{aligned}
& \left(a \sharp c_{g}\right)_{1}^{h}\left(\frac{x_{m}}{2}, \xi\right):=-\cos (\xi) \frac{1}{2 \pi} \sum_{n \in \mathbb{Z}} \partial_{x}^{h}\left(\mathcal{K}_{\gamma^{h}}^{h,+} \widehat{\widehat{a}}\right)\left(\frac{x_{m}}{2}, n\right) \exp (i \xi n), \\
& \left(a \sharp c_{g}\right)_{2}^{h}\left(\frac{x_{m}}{2}, \xi\right):=i \sin (\xi) \frac{1}{2 \pi} \sum_{n \in \mathbb{Z}} \mathfrak{M}_{x}^{h}\left(\mathcal{K}_{\gamma^{h}}^{h,-} \overline{\widehat{a}}\right)\left(\frac{x_{m}}{2}, n\right) \exp (i \xi n), \\
& \left(a \sharp c_{g}\right)_{3}^{h}\left(\frac{x_{m}}{2}, \xi\right):=2 \cos (\xi) \frac{1}{2 \pi} \sum_{n \in \mathbb{Z}} \mathfrak{M}_{x}^{h}\left(\mathcal{K}_{\delta^{h}}^{h,+} \widehat{\widehat{a}}\right)\left(\frac{x_{m}}{2}, n\right) \exp (i \xi n)
\end{aligned}
$$

and

$$
\left(a \sharp c_{g}\right)_{4}^{h}\left(\frac{x_{m}}{2}, \xi\right):=-i \sin (\xi) \frac{h^{2}}{2} \frac{1}{2 \pi} \sum_{n \in \mathbb{Z}} \partial_{x}^{h}\left(\mathcal{K}_{\delta^{h}}^{h,-} \widehat{\widehat{a}}\right)\left(\frac{x_{m}}{2}, n\right) \exp (i \xi n) .
$$

By the Taylor expansion A.5, we get $\mathcal{K}_{c}^{h,+}\left(x_{m \pm 1} / 2, n\right) \sim c\left(x_{m \pm 1} / 2\right)$ and $\mathcal{K}_{c}^{h,-}\left(x_{m \pm 1} / 2, n\right) \sim n c^{\prime}\left(x_{m \pm 1} / 2\right)$ as $h \rightarrow 0$. Moreover, by taking into account 3.12 , we obtain

$$
\left(a \sharp c_{g}\right)_{1}^{h}\left(\frac{x_{m}}{2}, \xi\right) \sim-\cos (\xi)\left(c_{g}^{\prime}\left(\frac{x_{m}}{2}\right) \bar{a}\left(\frac{x_{m}}{2}, \xi\right)+c_{g}\left(\frac{x_{m}}{2}\right) \partial_{x} \bar{a}\left(\frac{x_{m}}{2}, \xi\right)\right), \quad\left(a \sharp c_{g}\right)_{4}^{h}\left(\frac{x_{m}}{2}, \xi\right) \sim 0,
$$

and

$$
\left(a \sharp c_{g}\right)_{2}^{h}\left(\frac{x_{m}}{2}, \xi\right) \sim \sin (\xi) c_{g}^{\prime}\left(\frac{x_{m}}{2}\right) \partial_{\xi} \bar{a}\left(\frac{x_{m}}{2}, \xi\right), \quad\left(a \sharp c_{g}\right)_{3}^{h}\left(\frac{x_{m}}{2}, \xi\right) \sim \cos (\xi) c_{g}^{\prime}\left(\frac{x_{m}}{2}\right) \bar{a}\left(\frac{x_{m}}{2}, \xi\right) .
$$

Let us denote by $\langle\cdot, \cdot\rangle$ to be the duality product between $\mathcal{S}^{\prime}(\mathbb{R}) \times \mathcal{D}^{\prime}([-\pi, \pi])$ and $\mathcal{S}(\mathbb{R}) \times \mathcal{D}([-\pi, \pi])$. From 4.6 and 4.7, we obtain that $\mathcal{I}_{j}^{h}(t) \rightarrow \mathcal{I}_{j}(t)$ as $h \rightarrow 0$ for each $\mathcal{I}_{j}^{h}(t)$ in 4.5$), 1 \leq j \leq 4$, with

$$
\begin{gathered}
\mathcal{I}_{1}(t):=\left\langle\mathcal{W}(\cdot, t, \cdot),-\cos (\xi) \partial_{x}\left(c_{g}(x) a\right)\right\rangle, \quad \mathcal{I}_{2}(t):=\left\langle\mathcal{W}(\cdot, t, \cdot), \sin (\xi) c_{g}^{\prime}(x) a_{\xi}\right\rangle, \\
\mathcal{I}_{3}(t):=\left\langle\mathcal{W}(\cdot, t, \cdot), \cos (\xi) c_{g}^{\prime}(x) a\right\rangle \text { and } \mathcal{I}_{4}(t)=0 .
\end{gathered}
$$

We conclude the proof of Theorem 3.3 by passing the derivatives of $a$ to the accompanying factors in each $\mathcal{I}_{j}(t), 1 \leq j \leq 4$.

4.2. Proof of Theorem 3.5 concerning the discrete wave equation (1.7). We use similar arguments to the ones in the alternative proof of Theorem 2.2. Step I. Equation (4.11) of the Wigner transform matrix $\mathcal{W}^{h}\left[\mathbf{w}^{\mathbf{h}}\right]$ of the solution $\mathbf{w}^{\mathbf{h}}(t)$ of 3.25 . Set $\boldsymbol{\Theta}:=\boldsymbol{\Theta}_{1}+h \boldsymbol{\Theta}_{0}$, where $\boldsymbol{\Theta}_{0}, \boldsymbol{\Theta}_{1}$ are the ones in (3.25). For simplicity, during this proof, we use $\mathcal{W}^{h}$ to denote the Wigner transform matrix $\mathcal{W}^{h}\left[\mathbf{w}^{\mathbf{h}}\right]$. Using the definition 3.3 and the equation 3.25, we obtain that the Fourier coefficients of $\mathcal{W}^{h}$ with respect to $\xi$ verify the equation

$$
\partial_{t} \widehat{\mathcal{W}}^{h}\left(\frac{x_{m}}{2}, t, n\right)=\frac{1}{h} \mathbf{\Theta}^{h}\left(x, h \partial_{x}\right) \mathbf{w}_{\frac{m-n}{2}}(t) \otimes \mathbf{w}_{\frac{m+n}{2}}^{*}(t)+\mathbf{w}_{\frac{m-n}{2}}(t) \otimes\left(\frac{1}{h} \mathbf{\Theta}^{h}\left(x, h \partial_{x}\right) \mathbf{w}_{\frac{m+n}{2}}(t)\right)^{*} .
$$

We multiply equation 4.8 by $h \overline{\bar{a}}\left(x_{m} / 2, n\right) / 4 \pi$ and add in $m, n \in \mathbb{Z}$ such that $n \equiv m$, where $a \in \mathcal{S}(\mathbb{R}) \times$ $\mathcal{D}([-\pi, \pi])$. After writing explicitly how the pseudo-differential operator $\boldsymbol{\Theta}^{h}\left(x, h \partial_{x}\right)$ acts, we get

$$
\left\langle\partial_{t} \widehat{\mathcal{W}}^{h}(\cdot, t, \cdot), \overline{\bar{a}}_{h}=\mathbf{A}^{h}+\mathbf{B}^{h},\right.
$$

where

$$
\mathbf{A}^{h}:=\frac{1}{(2 \pi)^{2}} \frac{h^{2}}{2} \sum_{m \in \mathbb{Z}} \sum_{n \equiv m} \sum_{p \in \mathbb{Z}_{\Pi^{h}}} \int_{\frac{1}{h}} \Theta\left(\frac{x_{m-n}}{2}, h \xi\right) \mathbf{w}_{p}(t) \otimes \mathbf{w}_{\frac{m+n}{2}}^{*}(t) \overline{\bar{a}}\left(\frac{x_{m}}{2}, n\right) \exp \left(i \xi\left(x_{\frac{m-n}{2}}-x_{p}\right)\right) d \xi
$$


and

$$
\mathbf{B}^{h}:=\frac{1}{(2 \pi)^{2}} \frac{h^{2}}{2} \sum_{m \in \mathbb{Z}} \sum_{n \equiv m} \sum_{p \in \mathbb{Z}} \int_{\Pi^{h}} \frac{1}{h} \mathbf{w}_{\frac{m-n}{2}}(t) \otimes \mathbf{w}_{p}^{*}(t) \mathbf{\Theta}^{*}\left(\frac{x_{m+n}}{2}, h \xi\right) \overline{\bar{a}}\left(\frac{x_{m}}{2}, n\right) \exp \left(-i \xi\left(x_{\frac{m+n}{2}}-x_{p}\right)\right) d \xi .
$$

Here, $\Pi^{h}:=[-\pi / h, \pi / h]$. In both $\mathbf{A}^{h}$ and $\mathbf{B}^{h}$ we do the change of variable $\eta:=\xi h$. Moreover, in $\mathbf{A}^{h}$ and $\mathbf{B}^{h}$ we change $m$ by $m^{\prime}:=p+(m+n) / 2$ and $m^{\prime}:=p+(m-n) / 2$, respectively. In that case, we observe that $m \equiv n$ is equivalent to $m^{\prime}, n \in \mathbb{Z}$. In $\mathbf{B}^{h}$, we also change $p$ by $p^{\prime}:=m^{\prime}-p$. After renaming $m^{\prime}, p^{\prime}$ as $m, p$ and $\eta$ as $\xi$, we get

$$
\mathbf{A}^{h}:=\frac{1}{(2 \pi)^{2}} \frac{h}{2} \sum_{m, n, p \in \mathbb{Z}} \int_{-\pi}^{\pi} \frac{1}{h} \Theta\left(x_{m-n-p}, \xi\right) \mathbf{w}_{p}(t) \otimes \mathbf{w}_{m-p}^{*}(t) \overline{\bar{a}}\left(x_{m-\frac{n}{2}-p}, n\right) \exp (i \xi(m-2 p-n)) d \xi
$$

and

$$
\mathbf{B}^{h}:=\frac{1}{(2 \pi)^{2}} \frac{h}{2} \sum_{m, n, p \in \mathbb{Z}} \int_{-\pi}^{\pi} \frac{1}{h} \mathbf{w}_{p}(t) \otimes \mathbf{w}_{m-p}^{*}(t) \boldsymbol{\Theta}^{*}\left(x_{p+n}, \xi\right) \overline{\bar{a}}\left(x_{p+\frac{n}{2}}, n\right) \exp (i \xi(m-2 p-n)) d \xi .
$$

As in the continuous case, we set $y:=x_{m} / 2, y_{1}:=x_{m / 2-n-p}$ and $y_{2}:=x_{m / 2-p-n / 2}$ and consider the Taylor expansions $\mathrm{A} .16$ of $\boldsymbol{\Theta}\left(y \pm y_{1}, \xi\right)$ and $\overline{\bar{a}}\left(y \pm y_{2}, n\right)$ at $x=y$. We also decompose $\mathbf{A}^{h}$ and $\mathbf{B}^{h}$ as $\mathbf{A}^{h}:=\tilde{\mathbf{A}}^{h}+\boldsymbol{\mathcal { R }}_{\mathbf{A}}^{h}$ and $\mathbf{B}^{h}:=\mathbf{B}^{h}+\mathcal{R}_{\mathbf{B}}^{h}$, where $\tilde{\mathbf{A}}^{h}$ and $\tilde{\mathbf{B}}^{h}$ are sums of the same type as $\mathbf{A}^{h}$ and $\mathbf{B}^{h}$, retaining from the terms $\boldsymbol{\Theta}\left(y \pm y_{1}, \xi\right) \overline{\bar{a}}\left(y \pm y_{2}, n\right)$ in $\mathbf{A}^{h}$ and $\mathbf{B}^{h}$ only $\boldsymbol{\Theta}(y, \xi) \overline{\bar{a}}(y, n) \pm y_{2} \boldsymbol{\Theta}(y, \xi) \partial_{x} \overline{\bar{a}}(y, n) \pm y_{1} \partial_{x} \boldsymbol{\Theta}(y, \xi) \overline{\bar{a}}(y, n)$.

In what follows, we use the equivalent expression below of the discrete Wigner transform (3.3)

$$
\mathcal{W}^{\epsilon}\left[\mathbf{f}^{\mathbf{h}, 1}, \mathbf{f}^{\mathbf{h}, 2}\right]\left(\frac{x_{m}}{2}, \xi\right):=\frac{h}{2 \pi \epsilon} \sum_{n \in \mathbb{Z}} \mathbf{f}_{n}^{1} \otimes\left(\mathbf{f}_{m-n}^{2}\right)^{*} \exp \left(\frac{i x_{m-2 n} \xi}{\epsilon}\right) .
$$

As in the continuous case, we also take into account the fact that the term $x_{m / 2-p}$ appearing in both $y_{1}$ and $y_{2}$ yields $h \partial_{\xi} \mathcal{W}^{h} / 2 i$ when add in $p$, while $-x_{n} \partial_{x}^{\alpha} \overline{\bar{a}}(y, n)$ yields $h \partial_{\xi} \partial_{x}^{\alpha} a(y, \xi) / i$ when add in $n$ (with $\alpha=0$ or $\alpha=1)$. Thus, $\tilde{\mathbf{A}}^{h}:=\tilde{\mathbf{A}}_{1}^{h}+\tilde{\mathbf{A}}_{2}^{h}+\tilde{\mathbf{A}}_{3}^{h}$, where

$$
\tilde{\mathbf{A}}_{1}^{h}:=\frac{1}{h}\left\langle\boldsymbol{\Theta} \mathcal{W}^{h}(\cdot, t, \cdot), a\right\rangle_{h, \mathcal{D}^{\prime}, \mathcal{D}}, \quad \tilde{\mathbf{A}}_{2}^{h}:=-\frac{1}{2 i}\left\langle\partial_{\xi} \boldsymbol{\Theta} \mathcal{W}^{h}(\cdot, t, \cdot), \partial_{x} a\right\rangle_{h, \mathcal{D}^{\prime}, \mathcal{D}}
$$

and

$$
\tilde{\mathbf{A}}_{3}^{h}:=-\frac{1}{2 i}\left\langle\partial_{x} \boldsymbol{\Theta} \partial_{\xi} \mathcal{W}^{h}(\cdot, t, \cdot)+2 \partial_{x \xi}^{2} \boldsymbol{\Theta} \mathcal{W}^{h}(\cdot, t, \cdot), a\right\rangle_{h, \mathcal{D}^{\prime}, \mathcal{D}}
$$

and a similar expression for $\tilde{\mathbf{B}}^{h}$ in which the signs of the second and of the third term in $\tilde{\mathbf{A}}^{h}$ are changed, $\Theta$ and its derivatives are replaced by their conjugate transpose matrices and interchanged with the corresponding derivatives of the discrete Wigner transform matrix $\mathcal{W}^{h}$. Thus, similarly to the continuous case, by taking into account that $\boldsymbol{\Theta}=\boldsymbol{\Theta}_{1}+h \boldsymbol{\Theta}_{0}$ and that $\boldsymbol{\Theta}_{1}^{*}=-\boldsymbol{\Theta}_{1}$, we obtain the following equation for $\mathcal{W}^{h}$ :

$$
\begin{aligned}
\partial_{t} \mathcal{W}^{h} & =\frac{\boldsymbol{\Theta}_{1} \mathcal{W}^{h}-\mathcal{W}^{h} \boldsymbol{\Theta}_{1}}{h}+\left(\boldsymbol{\Theta}_{0} \mathcal{W}^{h}+\mathcal{W}^{h} \boldsymbol{\Theta}_{0}^{*}\right)-\frac{1}{2 i} \mathfrak{D}\left(\partial_{\xi} \boldsymbol{\Theta}_{1} \mathcal{W}^{h}+\mathcal{W}^{h} \partial_{\xi} \boldsymbol{\Theta}_{1}\right) \\
& -\frac{1}{2 i}\left(\partial_{x} \boldsymbol{\Theta}_{1} \partial_{\xi} \mathcal{W}^{h}+\partial_{\xi} \mathcal{W}^{h} \partial_{x} \boldsymbol{\Theta}_{1}\right)-\frac{1}{i}\left(\partial_{x \xi}^{2} \boldsymbol{\Theta}_{1} \mathcal{W}^{h}+\mathcal{W}^{h} \partial_{x \xi}^{2} \boldsymbol{\Theta}_{1}\right)+h \boldsymbol{\mathcal { R }}^{h}=: \sum_{j=1}^{5} \mathfrak{T}_{j}^{h}+h \boldsymbol{\mathcal { R }}^{h}
\end{aligned}
$$

where the operator $\mathfrak{D}$ (standing from derivative) is given by $\langle\mathfrak{D} f, a\rangle_{h, \mathcal{D}^{\prime}, \mathcal{D}}=\left\langle f, \partial_{x} a\right\rangle_{h, \mathcal{D}^{\prime}, \mathcal{D}}$ and $\mathcal{R}^{h}$ is bounded in $\mathcal{S}^{\prime}(\mathbb{R}) \times \mathcal{D}^{\prime}(\mathbb{R})$ as $h \rightarrow 0$.

Equation (4.22) of the projections of the Wigner measure matrix on the Fourier modes of $\boldsymbol{\Theta}_{1}$. As in the continuous case, we denote by $\mathcal{W}(x, t, \xi)$ to be the weak limit of the matrix $\mathcal{W}^{h}(x, t, \xi)$ and by $\mathcal{W}^{ \pm}$the weak limit of $\mathcal{W}^{h, \pm}:=\boldsymbol{\Delta}^{ \pm} \mathcal{W}^{h} \boldsymbol{\Delta}^{ \pm}$in (3.28), with $\boldsymbol{\Delta}^{ \pm}(\xi)$ being the projectors in (3.27). Passing to the limit in the term of order $h^{-1}$ in the right hand side of (4.11), we obtain

$$
\tilde{\boldsymbol{\Theta}}_{1}(x, \xi) \mathcal{W}(x, t, \xi)=\mathcal{W}(x, t, \xi) \tilde{\boldsymbol{\Theta}}_{1}(x, \xi), \quad \forall x \in \mathbb{R}, \xi \in[-\pi, \pi],
$$

where $\tilde{\boldsymbol{\Theta}}_{1}(x, \xi)$ is the limit as $h \rightarrow 0$ of $\boldsymbol{\Theta}_{1}(x, \xi)$ in 3.25 in which $\gamma^{h}(x)$ is replaced by $c_{g}(x)$ in 3.23 . 
Let us observe that the projectors $\boldsymbol{\Delta}^{ \pm}(\xi)$ in 3.27 verify the same properties 4 A.13 as in the continuous case. Both matrices $\boldsymbol{\Theta}_{1}$ and $\tilde{\boldsymbol{\Theta}}_{1}$ admit the decomposition A.12 with $\omega(\xi)=2 \sin (\xi / 2)$, while $c=\gamma^{h}$ in the first case and $c=c_{g}$ in the second one.

By multiplying by $\boldsymbol{\Delta}^{ \pm}$to the left and to the right in 4.11 , the left hand side becomes $\partial_{t} \mathcal{W}^{h, \pm}$, while the term of order $h^{-1}$ on the right hand side, $\boldsymbol{\Delta}^{ \pm} \boldsymbol{T}_{1}^{h} \boldsymbol{\Delta}^{ \pm}$, disappears due to identity A.22), in which $\omega(\xi)=2 \sin (\xi / 2)$ and $c=c_{g}$. It is easy to check that

$$
\begin{aligned}
\boldsymbol{\Delta}^{ \pm}(\xi) \boldsymbol{\Theta}_{0}(x, \xi) \boldsymbol{\Delta}^{ \pm}(\xi) & = \pm \frac{1}{2}\left(\alpha^{h}(x) \partial^{h,-} \beta^{h}(x)+\beta^{h}(x) \partial^{h,+} \alpha^{h}(x)\right) \cos (\xi / 2) \boldsymbol{\Delta}^{ \pm}(\xi) \\
& \pm \frac{1}{4}\left(\alpha^{h}(x) \partial^{h,-} \beta^{h}(x)-\beta^{h}(x) \partial^{h,+} \alpha^{h}(x)\right)(-2 i \sin (\xi / 2)) \boldsymbol{\Delta}^{ \pm}(\xi) .
\end{aligned}
$$

This is the analogue of A.24 in the continuous case. Taking into account (A.23), (A.26) and the approximations (3.23), we get:

$$
\boldsymbol{\Delta}^{ \pm}(\xi) \boldsymbol{T}_{2}^{h} \boldsymbol{\Delta}^{ \pm}(\xi) \sim \pm c_{g}^{\prime}(x) \cos (\xi / 2) \mathcal{W}^{ \pm}(x, t, \xi)
$$

as $h \rightarrow 0$, for all $x \in \mathbb{R}, t>0$ and $\xi \in[-\pi, \pi]$.

Since the projectors $\boldsymbol{\Delta}^{ \pm}(\xi)$ do not depend on $x$, the operator $\mathfrak{D}$ in the third term on the right hand side of 4.11 commutes with the projectors. Taking into account A.12 and A.13, we obtain

$$
\frac{1}{2 i} \boldsymbol{\Delta}^{ \pm} \partial_{\xi} \boldsymbol{\Theta}_{1} \mathcal{W}^{h} \boldsymbol{\Delta}^{ \pm}=\frac{1}{2} \partial_{\xi} \lambda_{\gamma^{h}, \omega}^{ \pm} \mathcal{W}^{h, \pm}+\frac{1}{2} \lambda_{\gamma^{h}, \omega}^{ \pm} \boldsymbol{\Delta}^{ \pm} \partial_{\xi} \boldsymbol{\Delta}^{ \pm} \mathcal{W}^{h} \boldsymbol{\Delta}^{ \pm}+\frac{1}{2} \lambda_{\gamma^{h}, \omega}^{\mp} \boldsymbol{\Delta}^{ \pm} \partial_{\xi} \boldsymbol{\Delta}^{\mp} \mathcal{W}^{h} \boldsymbol{\Delta}^{ \pm}
$$

By taking derivative in $\xi$ in the first identity in A.13 and multiplying the result to both sides by $\boldsymbol{\Delta}^{ \pm}$, we get

$$
\boldsymbol{\Delta}^{ \pm} \partial_{\xi} \boldsymbol{\Delta}^{ \pm} \boldsymbol{\Delta}^{ \pm}=\mathbf{0}_{2} \text { and } \boldsymbol{\Delta}^{ \pm} \partial_{\xi} \boldsymbol{\Delta}^{\mp} \boldsymbol{\Delta}^{ \pm}=\mathbf{0}_{2}
$$

Using A.13 and the definition of $\mathcal{W}^{h, \pm}:=\boldsymbol{\Delta}^{ \pm} \boldsymbol{W}^{h} \boldsymbol{\Delta}^{ \pm}$, the following identity holds:

$$
\boldsymbol{\Delta}^{ \pm} \partial_{\xi} \boldsymbol{\Delta}^{ \pm} \mathcal{W}^{h} \boldsymbol{\Delta}^{ \pm}=\boldsymbol{\Delta}^{ \pm} \partial_{\xi} \boldsymbol{\Delta}^{ \pm} \boldsymbol{\Delta}^{ \pm} \mathcal{W}^{h, \pm}+\boldsymbol{\Delta}^{ \pm} \partial_{\xi} \boldsymbol{\Delta}^{ \pm} \boldsymbol{\Delta}^{\mp} \mathcal{W}^{h} \boldsymbol{\Delta}^{ \pm}
$$

From (4.16), the first term on the right hand side of (4.17) vanishes, while from A.26) we get that the last one tends to zero as $h \rightarrow 0$ for $\xi \neq 0$. The last term in the right hand side of $(4.15)$ tends to zero by similar arguments. Thus,

$$
\left\langle\boldsymbol{\Delta}^{ \pm} \boldsymbol{T}_{3}^{h} \boldsymbol{\Delta}^{ \pm}, a\right\rangle_{h, \mathcal{D}^{\prime}, \mathcal{D}} \rightarrow-\left\langle\partial_{\xi} \lambda_{c_{g}, \omega}^{ \pm} \mathcal{W}^{ \pm}, \partial_{x} a\right\rangle
$$

as $h \rightarrow 0$ for any smooth test function $a$. By passing the derivative of $a$ to the accompanying factors, we get $-\left\langle\partial_{\xi} \lambda_{c_{g}, \omega}^{ \pm} \mathcal{W}^{ \pm}, \partial_{x} a\right\rangle=\left\langle\partial_{x \xi}^{2} \lambda_{c_{g}, \omega}^{ \pm} \mathcal{W}^{ \pm}+\partial_{\xi} \lambda_{c_{g}, \omega}^{ \pm} \partial_{x} \mathcal{W}^{ \pm}, a\right\rangle$.

Due to the fact that the projectors $\boldsymbol{\Delta}^{ \pm}$do not depend on the space variable $x$, but depend on $\xi$, we get the following two identities (we also use A.12 with $c=\gamma^{h}$ and $\omega(\xi)=2 \sin (\xi / 2)$ ):

$$
\boldsymbol{\Delta}^{ \pm} \boldsymbol{T}_{4}^{h} \boldsymbol{\Delta}^{ \pm}=-\partial_{x} \lambda_{\gamma^{h}, \omega}^{ \pm} \boldsymbol{\Delta}^{ \pm} \partial_{\xi} \mathcal{W}^{h} \boldsymbol{\Delta}^{ \pm}=-\partial_{x} \lambda_{\gamma^{h}, \omega}^{ \pm} \partial_{\xi} \mathcal{W}^{h, \pm}+\partial_{x} \lambda_{\gamma^{h}, \omega}^{ \pm}\left(\partial_{\xi} \boldsymbol{\Delta}^{ \pm} \mathcal{W}^{h} \boldsymbol{\Delta}^{ \pm}+\boldsymbol{\Delta}^{ \pm} \mathcal{W}^{h} \partial_{\xi} \boldsymbol{\Delta}^{ \pm}\right)
$$

and

$$
\begin{aligned}
\boldsymbol{\Delta}^{ \pm} \boldsymbol{T}_{5}^{h} \boldsymbol{\Delta}^{ \pm} & =-2 \partial_{x \xi}^{2} \lambda_{\gamma^{h}, \omega}^{ \pm} \mathcal{W}^{h, \pm}-\partial_{x} \lambda_{\gamma^{h}, \omega}^{ \pm}\left(\boldsymbol{\Delta}^{ \pm} \partial_{\xi} \boldsymbol{\Delta}^{ \pm} \mathcal{W}^{h} \boldsymbol{\Delta}^{ \pm}+\boldsymbol{\Delta}^{ \pm} \mathcal{W}^{h} \partial_{\xi} \boldsymbol{\Delta}^{ \pm} \boldsymbol{\Delta}^{ \pm}\right) \\
& -\partial_{x} \lambda_{\gamma^{h}, \omega}^{\mp}\left(\boldsymbol{\Delta}^{ \pm} \partial_{\xi} \boldsymbol{\Delta}^{\mp} \boldsymbol{W}^{h} \boldsymbol{\Delta}^{ \pm}+\boldsymbol{\Delta}^{ \pm} \mathcal{W}^{h} \partial_{\xi} \boldsymbol{\Delta}^{\mp} \boldsymbol{\Delta}^{ \pm}\right) .
\end{aligned}
$$

Using the third identity in A.13, we obtain the following two identities:

From 4.17 combined with A.26 and 4.16), we obtain that the last two terms in the right hand side of 4.20 tend to $\mathbf{0}_{2}$ as $h \rightarrow 0$.

From A.26, the second term in the right hand side of each identity in 4.21 tends to zero as $h \rightarrow 0$.

Thus, by passing to the limit as $h \rightarrow 0$ in 4.11, we obtain that each matrix $\mathcal{W}^{ \pm}$verifies the problem (with $\lambda_{c, \omega}^{ \pm}:= \pm c(x) \omega(\xi), \omega(\xi):=2 \sin (\xi / 2)$ and $c_{g}$ as in 3.23 )

$$
\partial_{t} \mathcal{W}^{ \pm}=\left\{\lambda_{c_{g}, \omega}^{ \pm}, \mathcal{W}^{ \pm}\right\}+\partial_{x} \lambda_{c_{g}, \omega}^{ \pm}\left(\partial_{\xi} \boldsymbol{\Delta}^{ \pm} \mathcal{W}^{ \pm}+\mathcal{W}^{ \pm} \partial_{\xi} \boldsymbol{\Delta}^{ \pm}\right)
$$


Here, $\{p, q\}:=\partial_{\xi} p \partial_{x} q-\partial_{\xi} q \partial_{x} p$ is the Poisson bracket introduced in A.18). Observe that, compared with A.29, there is an additional term in the right hand side of 4.22 , due to the fact that, in the discrete case, the projectors $\boldsymbol{\Delta}^{ \pm}$depend on the phase variable $\xi$.

In order to conclude 3.29 in Theorem 3.5 , we should prove that

$$
\operatorname{tr}\left(\partial_{\xi} \boldsymbol{\Delta}^{ \pm} \mathcal{W}^{ \pm}+\mathcal{W}^{ \pm} \partial_{\xi} \boldsymbol{\Delta}^{ \pm}\right)=0
$$

Writing explicitly both identities A.26 (corresponding to the + and the - sign) in term of the components of the matrix $\mathcal{W}$, we obtain that the components of $\mathcal{W}$ satisfy the following two identities

$$
W_{11}=W_{22} \text { and } W_{12} \exp (i \xi / 2)=W_{21} \exp (-i \xi / 2) .
$$

Intuitively, this means that, asymptotically, the kinetic and the potential energies of (1.7) propagate identically. Using these two identities, we can show that $\mathcal{W}^{ \pm}=\left(W_{11} \pm W_{12} \exp (i \xi / 2)\right) \boldsymbol{\Delta}^{ \pm}$. Thus, by deriving in $\xi$ the first identity in A.13, we obtain

$$
\begin{aligned}
\partial_{\xi} \boldsymbol{\Delta}^{ \pm} \boldsymbol{W}^{ \pm}+\mathcal{W}^{ \pm} \partial_{\xi} \boldsymbol{\Delta}^{ \pm} & =\left(W_{11} \pm W_{12} \exp (i \xi / 2)\right)\left(\partial_{\xi} \boldsymbol{\Delta}^{ \pm} \boldsymbol{\Delta}^{ \pm}+\boldsymbol{\Delta}^{ \pm} \partial_{\xi} \boldsymbol{\Delta}^{ \pm}\right) \\
& =\left(W_{11} \pm W_{12} \exp (i \xi / 2)\right) \partial_{\xi} \boldsymbol{\Delta}^{ \pm}
\end{aligned}
$$

Then 4.23 follows by observing that, since $\boldsymbol{\Delta}^{ \pm}$has constant elements on the main diagonal, $\partial_{\xi} \boldsymbol{\Delta}^{ \pm}$has trivial elements on the main diagonal. This concludes the proof of Theorem 3.5 .

\section{NUMERICAL EXPERIMENTS AND THEIR INTERPRETATION}

This section is devoted to present and interpret in terms of our theoretical results in Section 3 several numerical simulations for the following two equations:

- the transport one (1.1) with constant coefficient $\varrho=1$ on the finite space interval $[-1,1]$ with periodic boundary conditions $u(-1, t)=u(1, t)$ on the time interval $[0, T]$ with $T=5$. As numerical method, we use the finite difference approximation (1.6) with $u_{0}(t)=u_{N}(t)$ and $u_{1}(t)=u_{N+1}(t)$.

- the wave one (1.4) with constant coefficients $\rho=\sigma=1$ on the finite space interval $[-1,1]$ with homogeneous Dirichlet boundary conditions on the time interval $[0, T]$ with $T=5$. As numerical method, we use the finite difference approximation 1.7 with $u_{0}(t)=u_{N+1}(t)=0$.

Here, $h:=1 /(N+1)$ with $N=249$. Set $\mathbf{x}^{h}$ to be the uniform mesh of size $h$ of the interval $[-1,1]$. Let us define the following two non-uniform meshes (see Fig. 4)

$$
\mathbf{g}^{h, 1}:=\tan \left(\pi \mathbf{x}^{h} / 4\right) \text { and } \mathbf{g}^{h, 2}:=2 \sin \left(\pi \mathbf{x}^{h} / 6\right) \text {. }
$$

Both transformations $g$ map the interval $[-1,1]$ into itself. The two meshes $\mathbf{g}^{h, 1}$ and $\mathbf{g}^{h, 2}$ are symmetric with respect to zero. The first one is finer in the middle and coarser at the endpoints, while for the second one the situation is opposite. Both grids satisfy the regularity assumptions of Theorems 3.3 and 3.5 .

The time discretization is done by means of the centered scheme $\left(f^{n+1}-f^{n-1}\right) / 2 \delta t$ for the transport equation and by the leap-frog scheme $\left(f^{n+1}-2 f^{n}+f^{n-1}\right) / \delta t^{2}$ for the wave equation. Here, $f^{n}$ is an approximation of the function $f(t)$ at the time $t=n \delta t$. Since these times schemes are explicit, we use the Courant-Fiedrichs-Lewy (CFL) condition $\delta t=\mu h$, with $\mu=0.1$.

The initial data in (1.6) and (1.7) are constructed out of the following Gaussian profile with $\gamma=h^{-0.9}$ :

$$
G_{\gamma}(y)=\exp \left(-\gamma\left(g^{-1}(y)-g^{-1}\left(y_{0}\right)\right)^{2} / 2\right) \exp \left(i \xi_{0} g^{-1}(y) / h\right) \text {. }
$$

Here, $g^{-1}$ denotes the inverse of function $g$. For the transport equation 1.6 we consider the initial data $\mathbf{u}^{h, 0}:=G_{\gamma}\left(\mathbf{g}^{h}\right)$. In Figures 5 ( for $\mathbf{g}^{h}=\mathbf{g}^{h, 1}$ ) and 6 (for $\mathbf{g}^{h}=\mathbf{g}^{h, 2}$ ), we represent these solutions in the column on the left for the particular cases (a) $\left(\xi_{0}, y_{0}\right)=(3 \pi / 4,1 / 2)$, (c) $\left(\xi_{0}, y_{0}\right)=(3 \pi / 4,0)$, (e) $\left(\xi_{0}, y_{0}\right)=(\pi / 2,1 / 2)$ and $(\mathrm{g})\left(\xi_{0}, y_{0}\right)=(\pi / 2,0)$. In each subfigure on the right, we represent the corresponding generalized ray of Geometric Optics. For the transport equation with periodic boundary conditions, the generalized rays are an union of curves based on the solution of the system (3.8) obtained as follows. Firstly, since in this section we deal with the transport equation (1.6), we plot $g\left(x_{1}(t)\right)$ for $t \in\left[0, t_{1}\right]$, where $\left(x_{1}(t), \xi_{1}(t)\right)$ is the solution of (3.8) with initial data $x_{1}(0)=g^{-1}\left(y_{0}\right)$ and $\xi_{1}(0)=\xi_{0}\left(y_{0}, \xi_{0}\right.$ being the ones in (5.2) $)$ and $t_{1}$ is the first time when $g\left(x_{1}(t)\right)$ reaches one of the endpoints of the computational domain $[-1,1], y^{\star}$. Then, for $j \geq 2$, we plot $g\left(x_{j}(t)\right)$ for $t \in\left[t_{j-1}, t_{j}\right]$, where $\left(x_{j}(t), \xi_{j}(t)\right)$ is the solution of 3.8$)$ with initial data $x_{j}\left(t_{j-1}\right)=-y^{\star}$ (which 


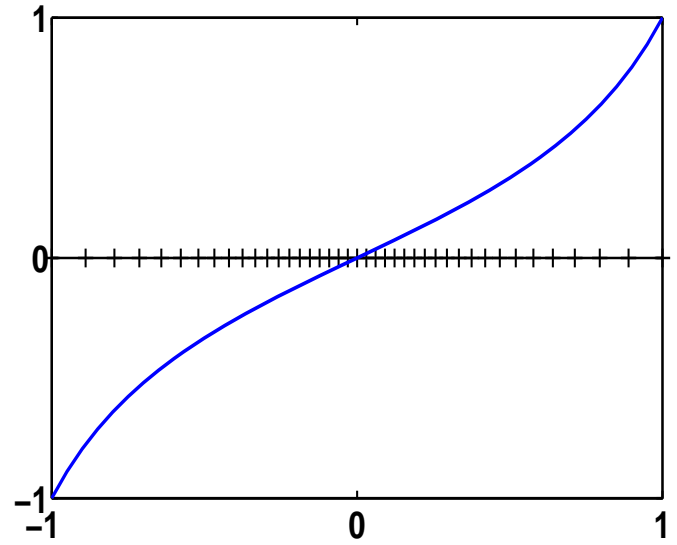

(a) Grid $\mathbf{g}^{h, 1}$

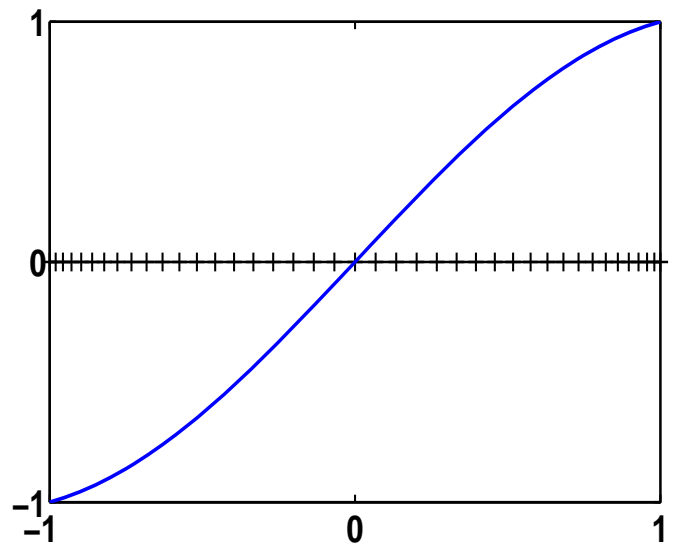

(b) Grid $\mathbf{g}^{h, 2}$

Figure 4. In black/blue, we represent the two non-uniform grids $\mathbf{g}^{h, 1}$ and $\mathbf{g}^{h, 2}$ (with markers indicating the grid points) and the application $g$ yielding the grid in each particular case.

is the opposite endpoint of $\left.y^{\star}\right)$ and $\xi_{j}\left(t_{j-1}\right)=\xi_{j-1}\left(t_{j-1}\right)$ and $t_{j}$ is the first time when $g\left(x_{j}(t)\right)$ reaches the endpoint $y^{\star}$. This iterative process finishes when $t=T$. The resolution of system (3.8) is done numerically by using the command ode 45 of the Matlab environment.

Let us denote by $\left(\lambda^{k}\right)_{-N \leq k \leq N}$ the square roots of the eigenvalues $\Lambda^{k}$ in the spectral problem below associated to the numerical approximation (1.7) for the wave equation (with constant coefficients $\sigma=\rho=1$ on the interval $(-1,1)$ with homogeneous Dirichlet boundary conditions) and by $\varphi^{h, k}=\left(\varphi_{j}^{k}\right)_{-N \leq j \leq N}$ the corresponding eigenvector:

$$
-\frac{1}{h}\left(\frac{\partial^{h,+} \varphi_{j}^{k}}{\partial^{h,+} g_{j}}-\frac{\partial^{h,-} \varphi_{j}^{k}}{\partial^{h,-} g_{j}}\right)=\Lambda^{k} \partial^{h} g_{j} \varphi_{j}^{k}, \quad-N \leq j \leq N, \quad \varphi_{-(N+1)}^{k}=\varphi_{N+1}^{k}=0 .
$$

The spectral decomposition of the solution of (1.7) is

$$
\mathbf{u}^{h}(t)=\sum_{ \pm} \sum_{-N \leq k \leq N}\left(\frac{1}{2}\left(\widehat{u}^{k, 0} \pm \frac{\widehat{u}^{k, 1}}{i \lambda^{k}}\right) \exp \left( \pm i t \lambda^{k}\right)\right) \varphi^{h, k}
$$

where $\widehat{u}^{k, 0}$ and $\widehat{u}^{k, 1}$ are the Fourier coefficients of the initial data $\mathbf{u}^{h, 0}$ and $\mathbf{u}^{h, 1}$ in 1.7 defined as $\widehat{u}^{k, i}:=$ $\left(\mathbf{u}^{h, i}, \varphi^{h, k}\right)_{\ell^{2}}, i=0,1$. Set $\widehat{G}_{\gamma}^{k}:=\left(G_{\gamma}\left(\mathbf{g}^{h}\right), \varphi^{h, k}\right)_{\ell^{2}}(-N \leq k \leq N)$ to be the Fourier coefficients of the vector $G_{\gamma}\left(\mathbf{g}^{h}\right)$.

We take the following initial data in (1.7)

$$
\mathbf{u}^{h, 0}:=\sum_{k=-N}^{N} \widehat{G}_{\gamma}^{k} \varphi^{h, k} \text { and } \mathbf{u}^{h, 1} \text { such that its Fourier coefficients are } \widehat{u}^{k, 1}:=i \lambda^{k} \widehat{u}^{k, 0} \text {. }
$$

Under the restrictions 5.5 , the solution of 1.7 becomes $\mathbf{u}^{h}(t):=\sum_{k=-N}^{N} \widehat{G}_{\gamma}^{k} \exp \left(i t \lambda^{k}\right) \varphi^{h, k}$.

In Figures 7 (for $\mathbf{g}^{h}=\mathbf{g}^{h, 1}$ ) and 8 (for $\mathbf{g}^{h}=\mathbf{g}^{h, 2}$ ), we represent these solutions in the column on the left for the particular cases $(\mathrm{a})\left(\xi_{0}, y_{0}\right)=(\pi, 1 / 2)$, (c) $\left(\xi_{0}, y_{0}\right)=(\pi, 0)$, (e) $\left(\xi_{0}, y_{0}\right)=(\pi / 2,1 / 2)$ and (g) $\left(\xi_{0}, y_{0}\right)=(\pi / 2,0)$. In each subfigure on the right, we represent the corresponding generalized ray of Geometric Optics. For the wave equation with homogeneous Dirichlet boundary conditions, the generalized rays consist of an union of curves based on the solution of (3.21) obtained by reflections on the boundary. More precisely, the first step $\mathbf{S}_{1}$ in the construction of the reflected ray is to plot $g\left(x_{1}^{+}(t)\right)$ for $t \in\left[0, t_{1}\right]$, where $\left(x_{1}^{+}(t), \xi_{1}^{+}(t)\right)$ is the solution of 3.21 (corresponding to the + sign) with initial data $x_{1}^{+}(0)=g^{-1}\left(y_{0}\right)$ and $\xi_{1}^{+}(0)=\xi_{0}\left(y_{0}, \xi_{0}\right.$ being the ones in $(5.2)$ ) and $t_{1}$ is the first time when $g\left(x_{1}^{+}(t)\right)$ reaches one of the 


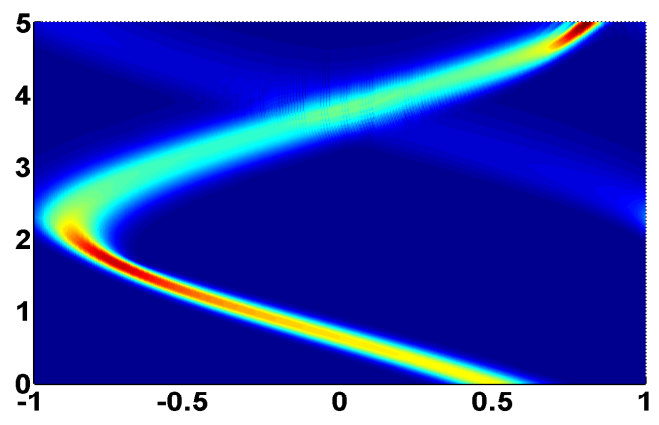

(a) Numerical solution for $\xi_{0}=3 \pi / 4, y_{0}=1 / 2$

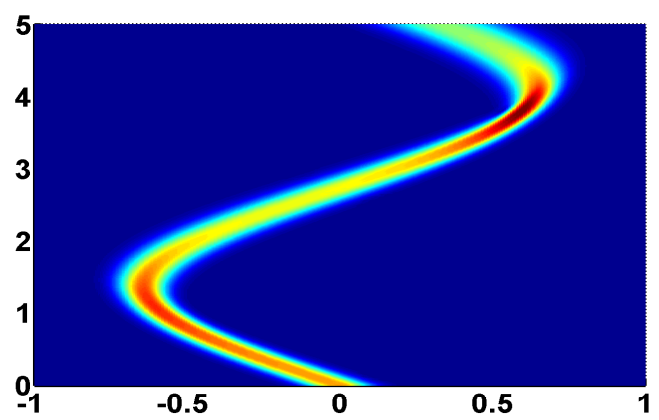

(c) Numerical solution for $\xi_{0}=3 \pi / 4, y_{0}=0$

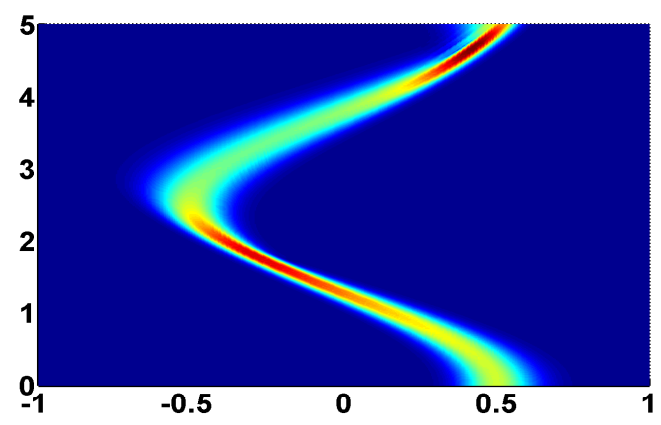

(e) Numerical solution for $\xi_{0}=\pi / 2, y_{0}=1 / 2$

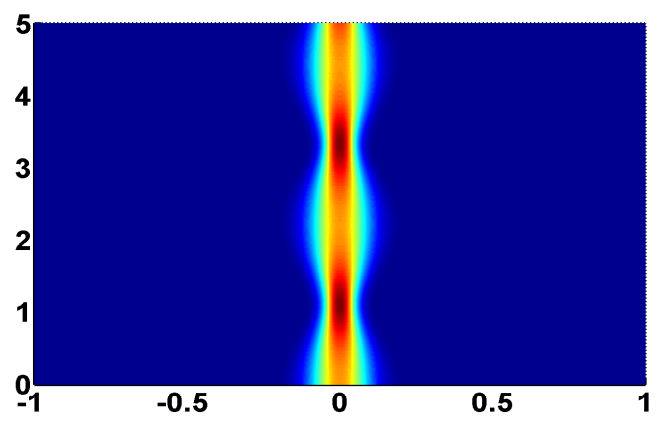

(g) Numerical solution for $\xi_{0}=\pi / 2, y_{0}=0$

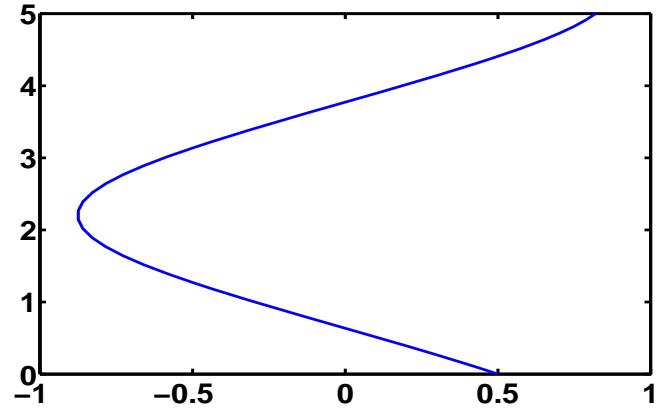

(b) The ray of Geometric Optics

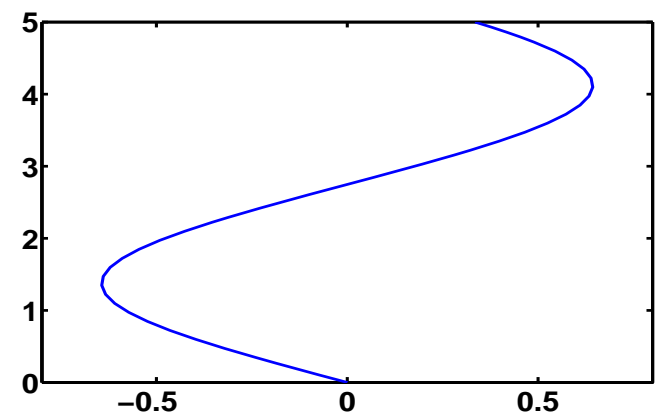

(d) The ray of Geometric Optics

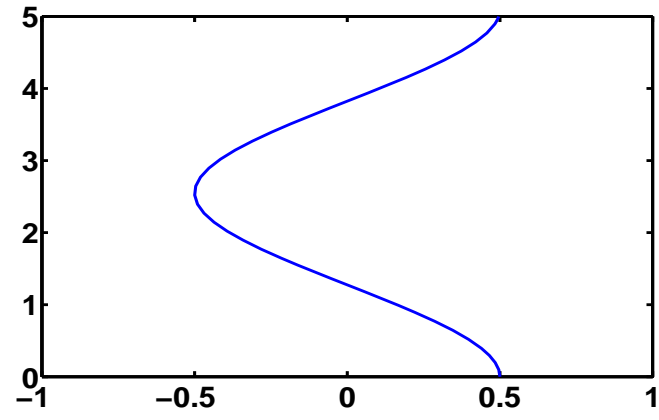

(f) The ray of Geometric Optics

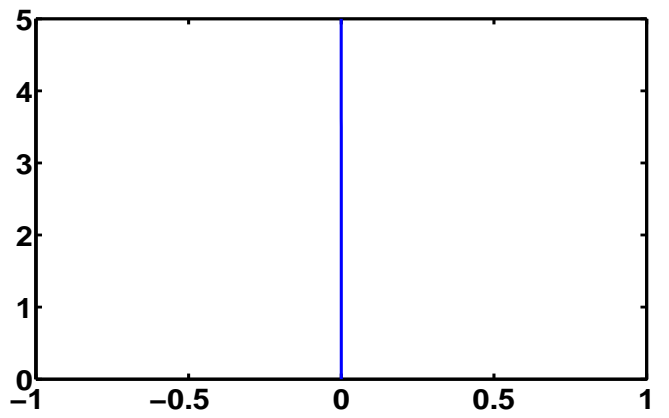

(h) The ray of Geometric Optics

FiguRE 5. The numerical solution of the transport equation (1.1) (with constant coefficient $\varrho=1)$ and the corresponding generalized ray, with $g(x)=\tan (\pi x / 4)$. 


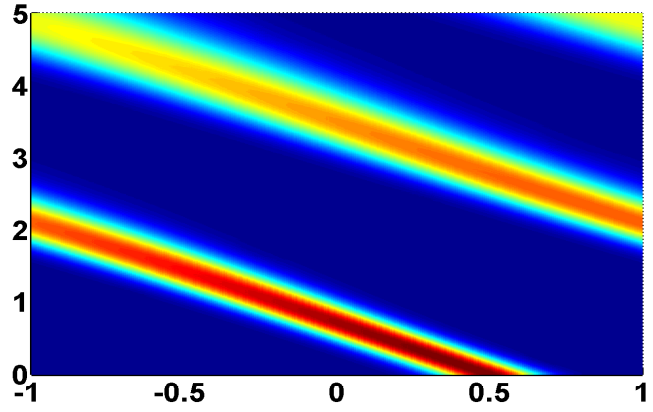

(a) Numerical solution for $\xi_{0}=3 \pi / 4, y_{0}=1 / 2$

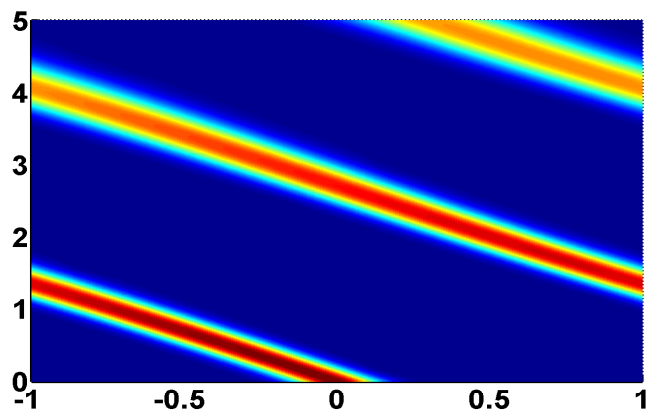

(c) Numerical solution for $\xi_{0}=3 \pi / 4, y_{0}=0$

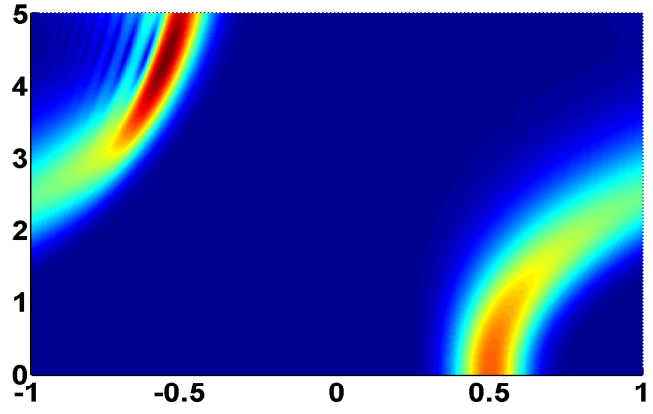

(e) Numerical solution for $\xi_{0}=\pi / 2, y_{0}=1 / 2$

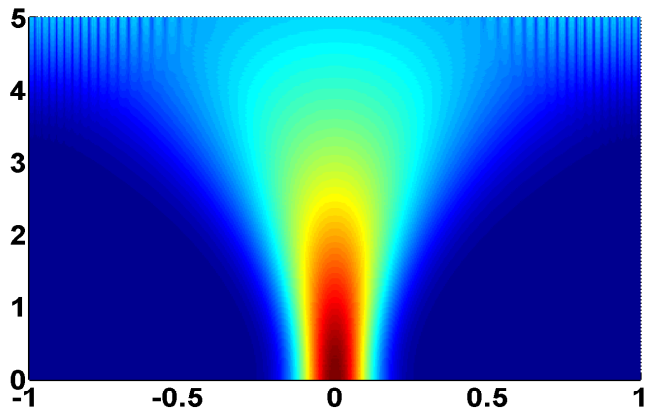

(g) Numerical solution for $\xi_{0}=\pi / 2, y_{0}=0$

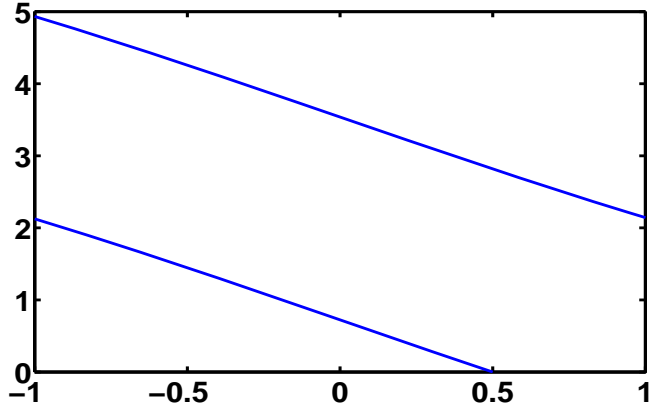

(b) The ray of Geometric Optics

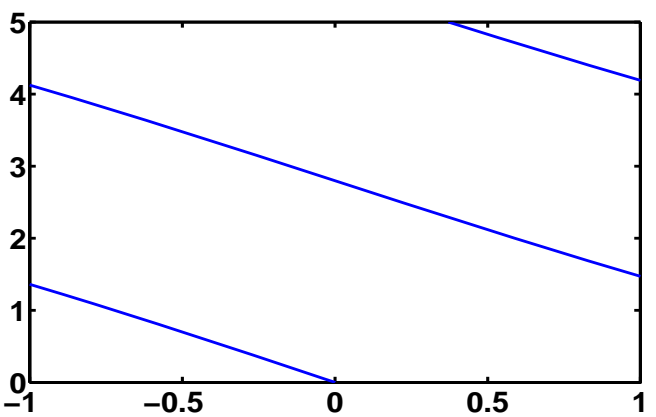

(d) The ray of Geometric Optics

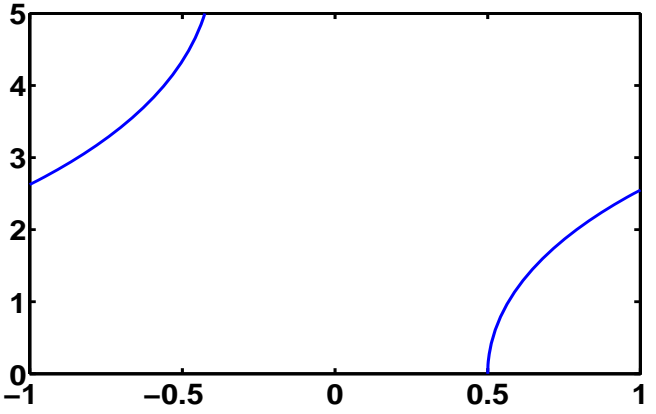

(f) The ray of Geometric Optics

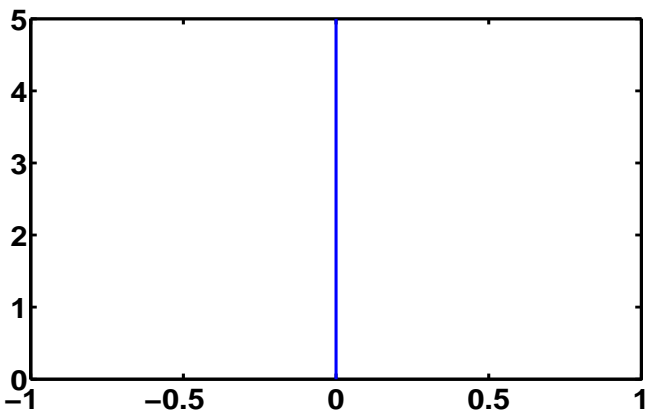

(h) The ray of Geometric Optics

FiguRE 6. The numerical solution of the transport equation (1.1) (with constant coefficient $\varrho=1)$ and the corresponding generalized ray, with $g(x)=2 \sin (\pi x / 6)$. 
endpoints of the computational domain $[-1,1], y^{\star}$. We chose the system (3.21) with the + sign at step $\mathbf{S}_{1}$ since in 5.5 we take the Fourier coefficients of the initial velocity $\mathbf{u}^{h, 1}$ of the form $\widehat{u}^{k, 1}=s(1) i \lambda^{k} \widehat{u}^{k, 0}$, with $s(1)=+$ being precisely the sign we chose at the first step. In general, we set $s(j)=-/+$ for even/odd $j$. At step $\mathbf{S}_{j}$, with $j \geq 2$, we plot $g\left(x_{j}^{s(j)}(t)\right)$ for $t \in\left[t_{j-1}, t_{j}\right]$, where $\left(x_{j}^{s(j)}(t), \xi_{j}^{s(j)}(t)\right)$ is the solution of 3.8 with $s(j)$ sign and initial data $x_{j}^{s(j)}\left(t_{j-1}\right)=s(j-1) y^{\star}$ and $\xi_{j}^{s(j)}\left(t_{j-1}\right)=\xi_{j-1}^{s(j-1)}\left(t_{j-1}\right)$ and $t_{j}$ is the first time when $g\left(x_{j}^{s(j)}(t)\right)$ reaches the endpoint $s(j) y^{\star}$. This process ends when $t=T$.

On the right column of Figures 5 /8, we represented $g\left(x^{ \pm}(t)\right)$, where $x^{ \pm}(t)$ is the space component of the solution of the coupled systems (3.8) or (3.21). Remark that $\left(y^{ \pm}(t)=g\left(x^{ \pm}(t)\right), \xi^{ \pm}(t)\right)$ satisfies the system

$$
\left(y^{ \pm}\right)^{\prime}(t)=\mp a_{g}\left(y^{ \pm}(t)\right) \omega^{\prime}\left(\xi^{ \pm}(t)\right), \quad\left(\xi^{ \pm}\right)^{\prime}(t)= \pm b_{g}\left(y^{ \pm}(t)\right) \omega\left(\xi^{ \pm}(t)\right), \quad y^{ \pm}(0)=y_{0}, \quad \xi^{ \pm}(0)=\xi_{0} .
$$

Here, $a_{g}(y):=\left(g^{\prime} c_{g}\right)\left(g^{-1}(y)\right)$ and $b_{g}(y):=c_{g}^{\prime}\left(g^{-1}(y)\right)$, where $c_{g}$ is as in 3.8 or as in 3.21). For the discrete transport equation, the symbol $\omega$ is given by $\omega(\xi):=\sin (\xi)$, while for discrete waves $\omega(\xi)=2 \sin (\xi / 2)$. When we deal with numerical schemes for the constant coefficients transport and wave equations on non-uniform meshes as in this section, the functions $a_{g}$ and $b_{g}$ in (5.6) take the simpler form

$$
a_{g}(y):=1 \text { and } b_{g}(y):=\left(\frac{1}{g^{\prime}}\right)^{\prime}\left(g^{-1}(y)\right) \text {. }
$$

Set $\mathbf{f}^{ \pm}(y, \xi):=\left(\mp \omega^{\prime}(\xi), \pm b_{g}(y) \omega(\xi)\right)$ (with $b_{g}$ as in (5.7)) and observe that (5.6) can be written as

$$
\left(\left(y^{ \pm}\right)^{\prime}(t),\left(\xi^{ \pm}\right)^{\prime}(t)\right)=\mathbf{f}^{ \pm}\left(y^{ \pm}(t), \xi^{ \pm}(t)\right), \quad y^{ \pm}(0)=y_{0}, \xi^{ \pm}(0)=\xi_{0} .
$$

5.1. High frequency pathologies. Let us point out the following three pathologies of the solutions of the discrete transport and wave equations and of the rays of Geometric Optics that we observe in Figures 5 8 .

- Non-propagating waves. In Figures $5 / 6$ (g), (h) and 7 8 (c),(d), we observe waves/rays that do not propagate. As we will see from the phase portrait analysis, they correspond to equilibrium (fixed) points $\left(y^{\sharp}, \xi^{\sharp}\right)$ (the green ones) on the corresponding phase diagrams in Figures 910 . However, there is a big difference concerning the dispersion along the ray between Fig.5. (g) and Fig 6 . $(\mathrm{g})$ and between Fig/7.(c) and Fig 8 (c). When the waves is very dispersive, the equilibrium point is a saddle point, whereas when the wave is not dispersive, the fixed point is a center. These non-propagating solutions are well-known to hold for numerical schemes on uniform meshes at wave numbers $\xi^{\sharp}$ where the group velocity $\omega^{\prime}\left(\xi^{\sharp}\right)$ vanishes.

- Trapped rays in the interior of the domain or at one endpoint. It is well-known that, for the continuous case or for numerical waves on uniform meshes concentrated on frequencies where the group velocity is not trivial, all the generalized rays are straight lines reflecting at both endpoints. Instead, when the mesh is non-uniform, the corresponding rays are in general strongly curved (see

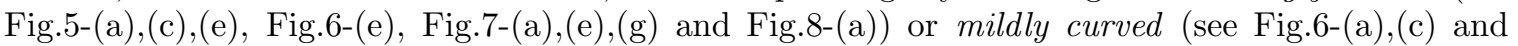
Fig 8 (e),(g)). The completely new pathology of the waves on non-uniform meshes is the presence of i) waves oscillating in the interior of the computational domain without reflections on the boundary (see Fig 5-(a),(c),(e) and Fig.7-(a),(e),(g)) and ii) waves which oscillate in the interior of the domain and reflect only at one of the endpoints (see Fig.8.(a)). The trajectories of these trapped rays always remain in the red area of the phase portraits in Figures 9 10. More precisely, the situation i) corresponds to periodic orbits in the phase diagram which are completely included in the region between the two dotted black vertical asymptotes indicating the computational domain $[-1,1]$, while ii) is related to the red lateral area limited by separatrices and the dotted black vertical asymptotes in Figures 910 . (b). Recall that a saddle point $O$ is characterized by the fact that the space around it is divided in four sectors by two curves (the separatrices) passing by $O$. Moreover, in each of the four sectors the trajectories are arcs of hyperbolas of center $O$ (cf. [16]).

- Generalized rays starting in the non-physical sense. The solutions for the continuous transport equation (1.1) with constant coefficient $\varrho=1$ and + sign are of the form $u(y, t)=u^{0}(y-t)$, so that they propagate to the right. The solutions of the wave equation 1.4 with constant coefficients $\rho=\sigma=1$ and initial data $u^{1}(y)=\left(u^{0}\right)^{\prime}(y)$ (which is the analogue of the second condition in (5.5)) are of the form $u(y, t)=u^{0}(y+t)$, so that they propagate to the left. These directions in the continuous case coincide with the arrow sense in the phase portraits in Figures $9+10$ at frequency $\xi=0$. 
However, as it could be observed in Fig/5-(a),(c),(e), Fig,6 (a),(c) and Fig,8 (a), the high frequency discrete waves could start in the non-physical sense. But in Fig 9 for $\xi \in[\pi / 2,3 \pi / 2]$ and in Fig. 10 for $\xi \in[\pi, 2 \pi]$ we see that the arrow orientation changes. This is not a new phenomenon for the discrete transport equation since when the grid is uniform the group velocity $\omega^{\prime}(\xi)=\cos (\xi)$ changes the sign for $\xi \in[\pi / 2,3 \pi / 3]$ and this yields waves propagating to the left. But this pathology of having waves moving in the non-physical sense is completely new for the finite-difference approximation of the wave equation on uniform meshes where the group velocity $\omega^{\prime}(\xi)=\cos (\xi / 2)$ does not change the sign for $\xi \in[-\pi, \pi]$.

5.2. Phase diagram analysis. The following energy of the solutions $\left(y^{ \pm}(t), \xi^{ \pm}(t)\right)$ of $(5.8)$ is conserved along trajectories:

$$
\mathrm{E}_{g, \omega}(t):=\frac{1}{g^{\prime}}\left(g^{-1}\left(y^{ \pm}(t)\right)\right) \omega\left(\xi^{ \pm}(t)\right) .
$$

The equilibrium (fixed) points $\left(y^{\sharp}, \xi^{\sharp}\right)$ of the phase portrait of the solution $\left(y^{ \pm}(t), \xi^{ \pm}(t)\right)$ satisfy $\mathbf{f}^{ \pm}\left(y^{\sharp}, \xi^{\sharp}\right)=$ $(0,0)$, so that $\left(y^{\sharp}, \xi^{\sharp}\right)$ is the solution of

$$
\omega^{\prime}\left(\xi^{\sharp}\right)=0 \text { and } b_{g}\left(y^{\sharp}\right)=0,
$$

with $b_{g}$ as in 5.7). From the condition of the second component $\mathbf{f}^{h}\left(y^{\sharp}, \xi^{\sharp}\right)$ to vanish, we should normally obtain an alternative equation of $b_{g}\left(y^{\sharp}\right)=0$ which is $\omega\left(\xi^{\sharp}\right)=0$. But since the symbol $\omega(\xi)$ is a trigonometrical one, it is in general difficult to guarantee simultaneously $\omega\left(\xi^{\sharp}\right)=\omega^{\prime}\left(\xi^{\sharp}\right)=0$.

The first equation in 5.10 yields precisely the wave numbers $\xi^{\sharp}$ where the group velocity $\omega^{\prime}(\xi)$ for the same numerical scheme on the uniform mesh vanishes, so that, for the finite difference approximation of the transport equation we get $\xi^{\sharp} \in\{(2 k+1) \pi / 2, k \in \mathbb{Z}\}$, while for the numerical wave equation $\xi^{\sharp} \in$ $\{(2 k+1) \pi, k \in \mathbb{Z}\}$. Thus, within the frequency range $\xi \in[0,2 \pi]$ for the phase portraits in Figures 9$] 10$, we obtain $\xi^{\sharp} \in\{\pi / 2,3 \pi / 2\}$ for the transport equation and $\xi^{\sharp}=\pi$ for the wave equation.

For the tangential mesh $g(x)=\tan (\pi x / 4)$, we get $b_{g}(g(x))=-\sin (\pi x / 2)$ which vanishes at any $x^{\sharp} \in 2 \mathbb{Z}$. The points $y^{\sharp}$ in (5.10) satisfy $y^{\sharp}=g\left(x^{\sharp}\right)$. For $x^{\sharp}=-2,0,2$ and $g(x)=\tan (\pi x / 4)$, we get $y^{\sharp}=-\infty, 0, \infty$. For the sinusoidal mesh $g(x)=2 \sin (\pi x / 6)$, we get $b_{g}(g(x))=\sin (\pi x / 6) /\left(2 \cos ^{2}(\pi x / 6)\right)$, which vanishes for any $x^{\sharp} \in 6 \mathbb{Z}$, so that $y^{\sharp}=0$, for each $x^{\sharp} \in 6 \mathbb{Z}$. Consequently, on both tangential and sinusoidal meshes, there are two fixed points for the transport equation, $\left(y^{\sharp}, \xi^{\sharp}\right)=(0, \pi / 2)$ and $\left(y^{\sharp}, \xi^{\sharp}\right)=(0,3 \pi / 2)$, while for the wave equation there is an unique equilibrium point, $\left(y^{\sharp}, \xi^{\sharp}\right)=(0, \pi)$.

However, in the sinusoidal case, $b_{g}(g(x))$ blows-up for $x^{b} \in 6 \mathbb{Z}+3$, yielding $y^{b}=g\left(x^{b}\right)=2(-1)^{k}$ for $x^{b}=3(2 k+1), k \in \mathbb{Z}$. Thus, $b_{g}$ is $C^{0,1}(-2+\delta, 2-\delta)$ for each $\delta>0$. On the phase portraits in Fig 9 (b), the points $(y, \xi) \in(-2, \pi),(2,0),(2,2 \pi)$ and $(y, \xi) \in(2, \pi),(-2,0),(-2,2 \pi)$ seem to be (attracting) stable and (repulsive) unstable nodes, respectively. The same happens with $(y, \xi) \in\{(-2,0),(2,2 \pi)\}$ and $(y, \xi) \in\{(2,0),(-2,2 \pi)\}$ in Fig 10 -(b). But we cannot find anyone of these points as solutions of the corresponding $\mathbf{f}^{ \pm}(y, \xi)=0$ (with $\mathbf{f}^{ \pm}$as in (5.8)). Moreover, in 31, p.160 we see that a conservative system cannot have any attracting point. But in [31, the author assumes that $\mathbf{f}$ is Lipschitz with respect to both components $(y, \xi)$. However, a function is not Lipschitz at its blow-up points, so that there is a lack of regularity for $b_{g}$ in the case of the sinusoidal mesh. Nevertheless, $b_{g}$ has the required regularity $C^{0,1}$ on $[-1,1]$.

In order to see the nature of the stable points of system, let us firstly observe that the Jacobian matrix of $\mathbf{f}^{ \pm}$is as follows:

$$
\mathbf{J f}^{ \pm}(y, \xi):=\left(\begin{array}{cc}
0 & \mp \omega^{\prime \prime}(\xi) \\
\pm b_{g}^{\prime}(y) \omega(\xi) & \pm b_{g}(y) \omega^{\prime}(\xi)
\end{array}\right)
$$

However, due to 5.10$)$, the component $(2,2)$ of the matrix $\mathbf{J f}^{ \pm}$at any fixed point $\left(y^{\sharp}, \xi^{\sharp}\right)$ vanishes, so that the two eigenvalues of $\mathbf{J f}^{ \pm}\left(y^{\sharp}, \xi^{\sharp}\right)$ are solutions of the quadratic equation:

$$
\lambda^{2}+b_{g}^{\prime}\left(y^{\sharp}\right) \omega\left(\xi^{\sharp}\right) \omega^{\prime \prime}\left(\xi^{\sharp}\right)=0 .
$$

For both numerical transport and wave equations and for any $\xi^{\sharp}$ satisfying 5.10 , we get $\omega\left(\xi^{\sharp}\right) \omega^{\prime \prime}\left(\xi^{\sharp}\right)=$ -1 , so that the eigenvalues $\lambda$ in 5.11 do not depend on the equation type (i.e. transport or waves). For 


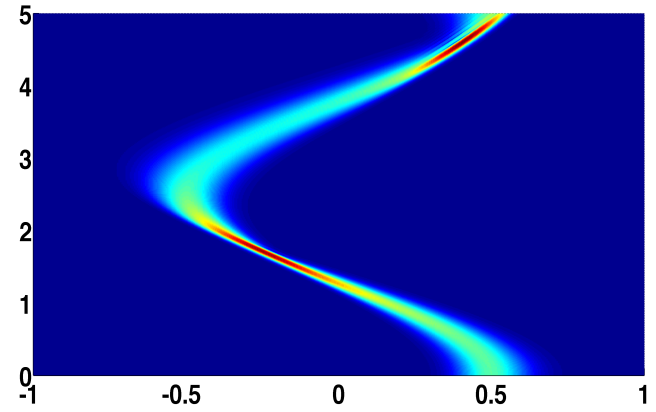

(a) Numerical solution for $\xi_{0}=\pi, y_{0}=1 / 2$

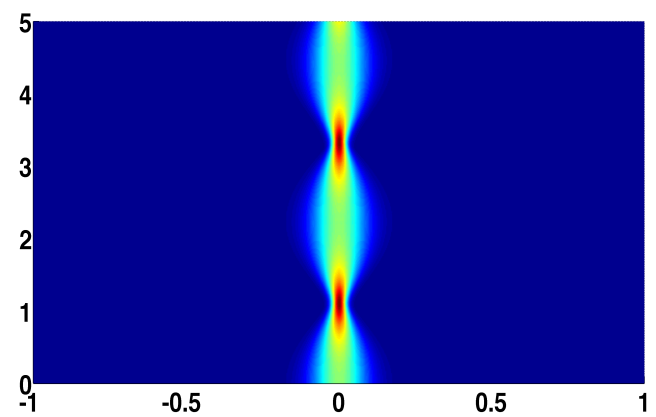

(c) Numerical solution for $\xi_{0}=\pi, y_{0}=0$

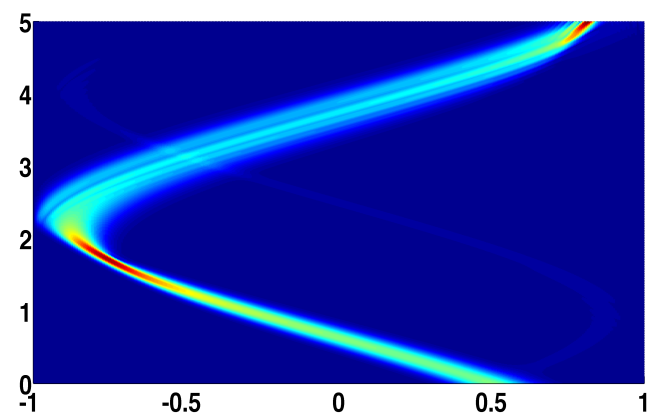

(e) Numerical solution for $\xi_{0}=\pi / 2, y_{0}=1 / 2$

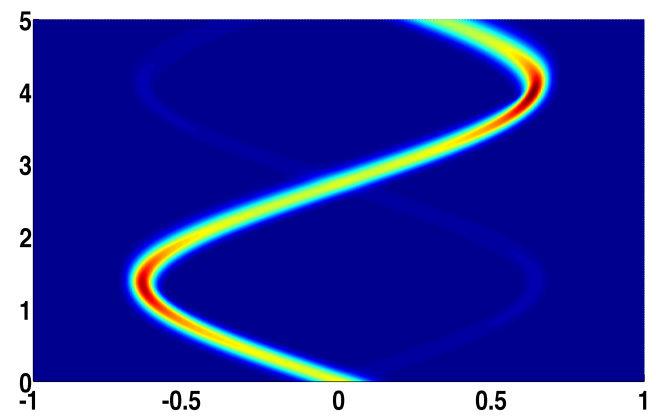

(g) Numerical solution for $\xi_{0}=\pi / 2, y_{0}=0$

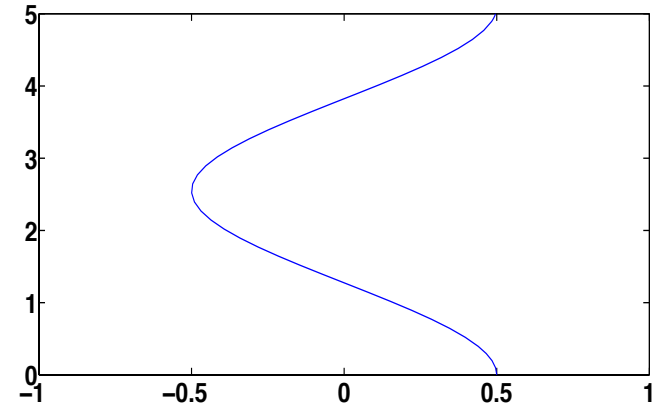

(b) The ray of Geometric Optics

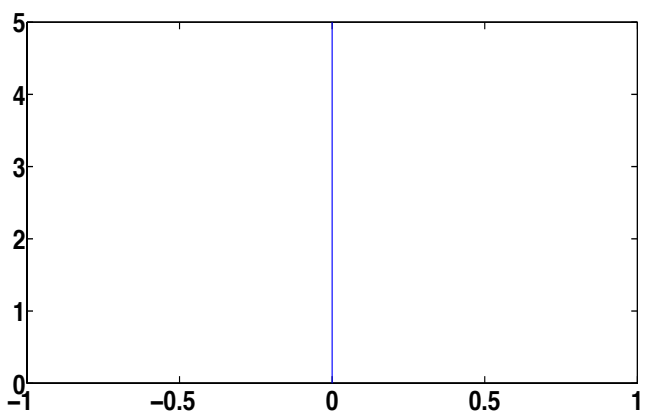

(d) The ray of Geometric Optics

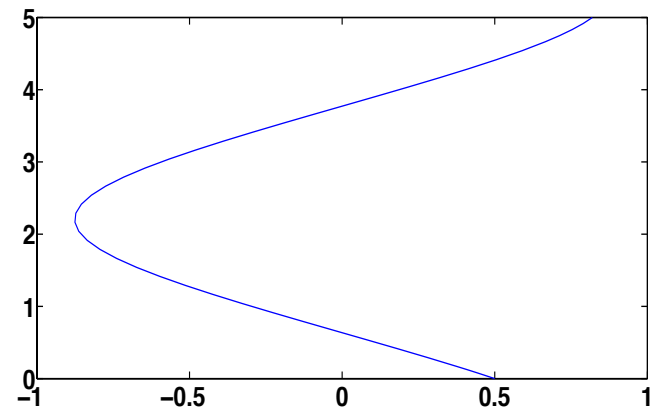

(f) The ray of Geometric Optics

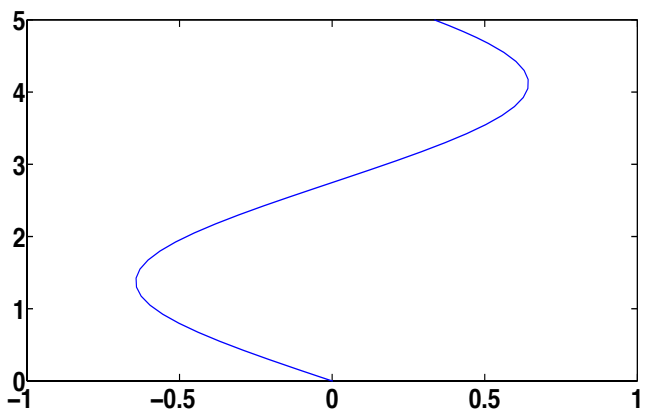

(h) The ray of Geometric Optics

Figure 7. The numerical solution of the wave equation (1.4) (with constant coefficient $\rho=\sigma=1)$ and the corresponding generalized ray, with $g(x)=\tan (\pi x / 4)$. 


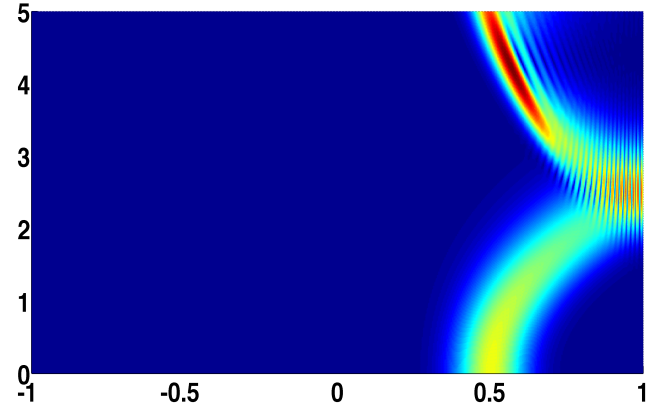

(a) Numerical solution for $\xi_{0}=\pi, y_{0}=1 / 2$

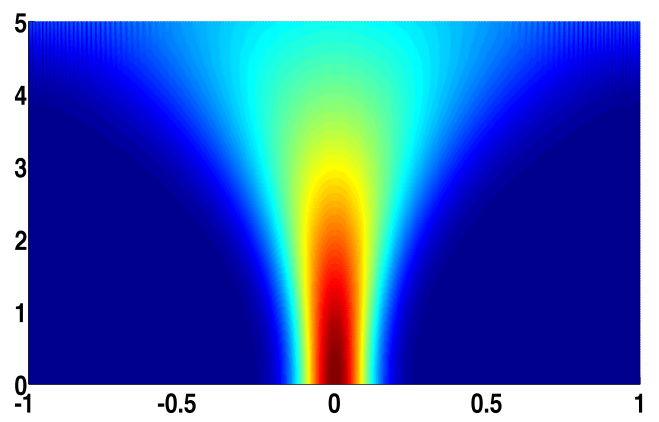

(c) Numerical solution for $\xi_{0}=\pi, y_{0}=0$

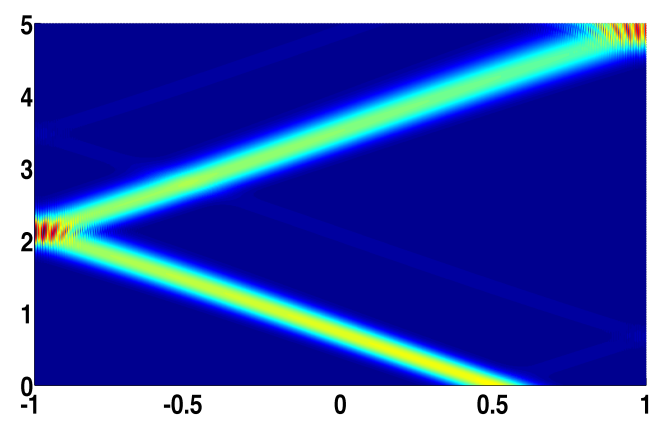

(e) Numerical solution for $\xi_{0}=\pi / 2, y_{0}=1 / 2$

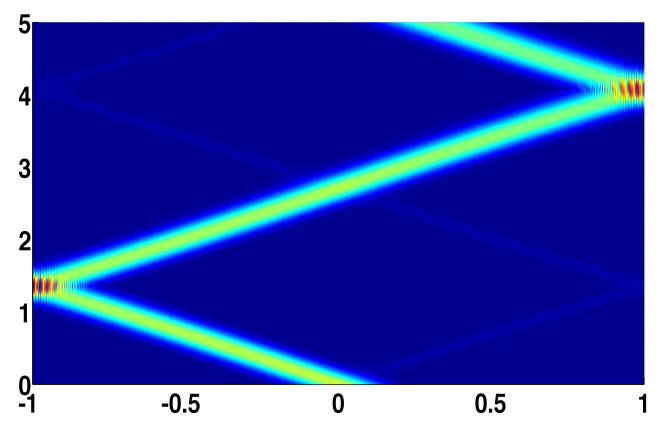

(g) Numerical solution for $\xi_{0}=\pi / 2, y_{0}=0$

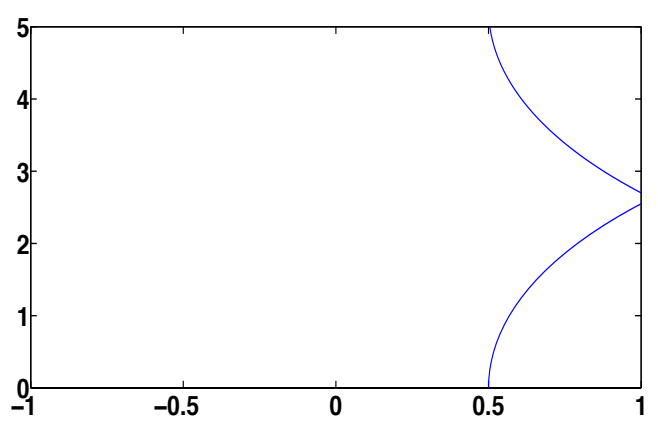

(b) The ray of Geometric Optics

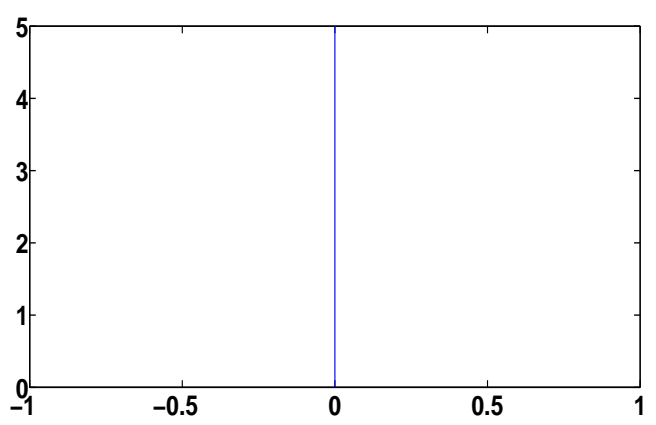

(d) The ray of Geometric Optics

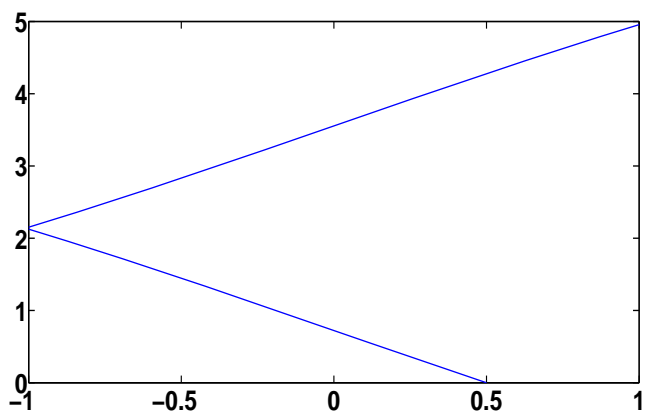

(f) The ray of Geometric Optics

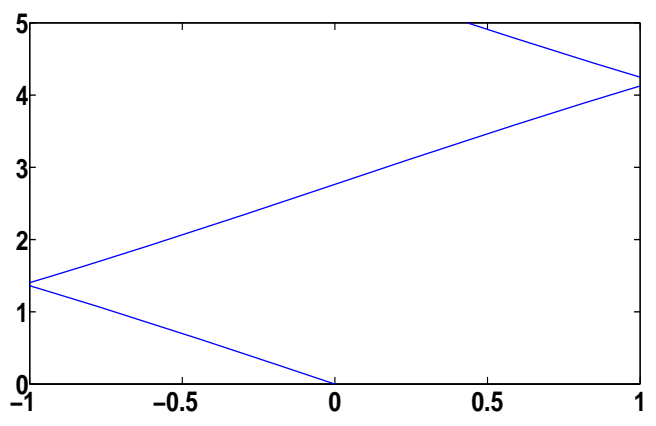

(h) The ray of Geometric Optics

Figure 8. The numerical solution of the wave equation 1.4 (with constant coefficient $\rho=\sigma=1)$ and the corresponding generalized ray, with $g(x)=2 \sin (\pi x / 6)$. 


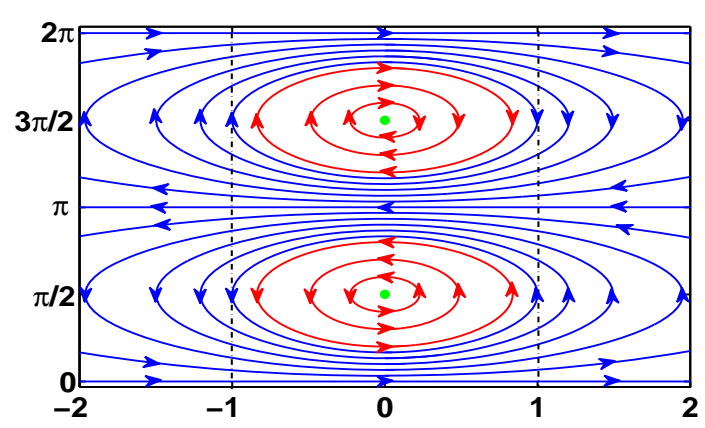

(a) $\mathbf{g}^{h, 1}$

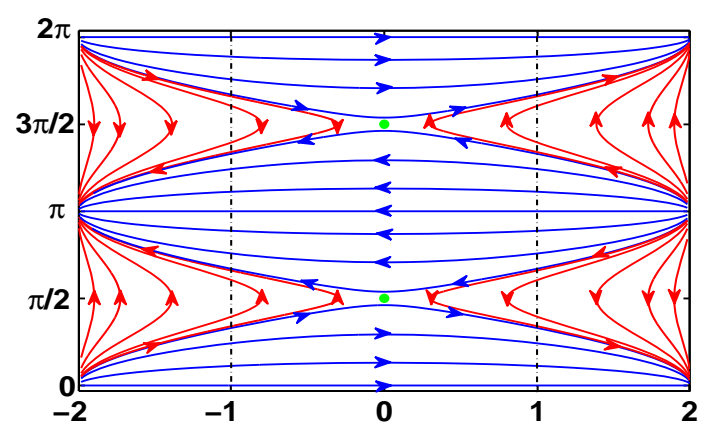

(b) $\mathbf{g}^{h, 2}$

Figure 9. The phase portrait of the system (5.8) for the numerical transport equation (i.e. $\omega(\xi)=\sin (\xi))$ and the grid transformations $g(x)=\tan (\pi x / 4)$ (left) and $g(x)=2 \sin (\pi x / 6)$ (right). We put $y^{-}(t), \xi^{-}(t)$ on the horizontal/vertical direction.

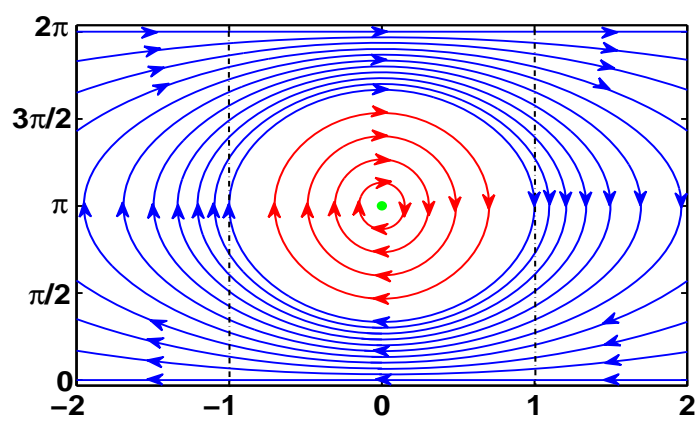

(a) $\mathrm{g}^{h, 1}$

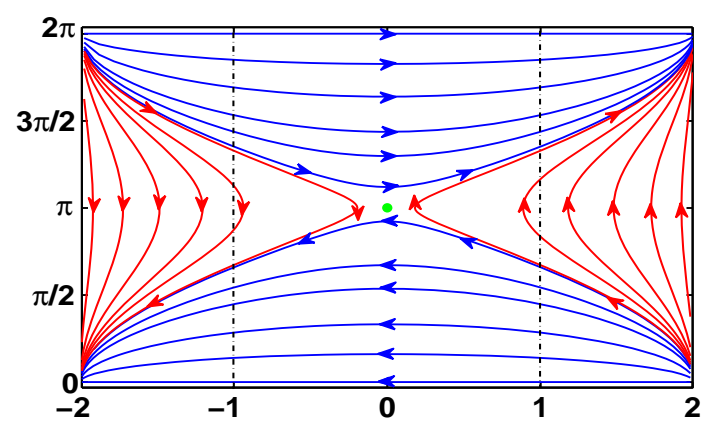

(b) $\mathrm{g}^{h, 2}$

Figure 10. The phase portrait of the system (5.8) for the numerical wave equation (i.e. $\omega(\xi)=2 \sin (\xi / 2))$ and the grid transformations $g(x)=\tan (\pi x / 4)$ (left) and $g(x)=2 \sin (\pi x / 6)$ (right). We put $y^{+}(t), \xi^{+}(t)$ on the horizontal/vertical direction.

both grid applications $g$ considered in this section, $y^{\sharp}=0$. Thus, for the tangential grid, we obtain a negative $b_{g}^{\prime}(0)(=-2)$, while for the sinusoidal one, we get a positive $b_{g}(0)(=1 / 4)$.

In the tangential case, the two eigenvalues in $\sqrt{5.11}$ are purely imaginary, $\lambda^{ \pm}= \pm \sqrt{2} i$, so that the equilibrium point is not a hyperbolic one in the sense of Chapter 7 in [29] and the Hartman-Grobman Theorem (cf. 29]) cannot be applied directly. However, due to the conservation of the energy $\mathrm{E}_{g, \omega}(t)$ in $(5.9)$ along the trajectories, the qualitative behavior of the linearized system at the fixed points still coincides with the one of the nonlinear one (5.8) (cf. Section 6.5 in [31]). Thus, for both transport and wave equations on the tangential mesh, all the equilibrium points $\left(y^{\sharp}, \xi^{\sharp}\right)$ in $(5.10$ are centers (and the trajectories around them are periodic orbits).

In the sinusoidal case, the two eigenvalues in (5.11) are real and of opposite signs, $\lambda^{ \pm}= \pm 1 / 2$, so that the equilibrium point is a hyperbolic one and the Hartman-Grobman Theorem (cf. [29]) works. Thus, for both transport and wave equations on the sinusoidal mesh, all the fixed points $\left(y^{\sharp}, \xi^{\sharp}\right)$ are of saddle type. 


\section{Comments And OPEn PRoblems}

In this article we have developed a microlocal approach for the analysis of the propagation properties of solutions of $1-d$ heterogeneous wave equations under non-unform finite difference discretizations. We have seen that a careful study of the phase portrait of the Hamiltonian system giving the characteristic rays indicates with precision important qualitative properties of the rays (their possibility to reach the boundary in finite time, or to have a stationary trajectory or to be reflected inside the domain when the mesh becomes to coarse to resolve the corresponding wave number, etc.). However, compared to our understanding on wave propagation in homogeneous media, there are plenty of phenomena to be understood of which we list the following ones:

1. Irregular meshes. Our analysis is limited to the case of smooth non-uniform meshes that can be obtained by diffeomorphic transformations of an uniform one. Very likely, in the case of more irregular meshes, obtained as deformations of the uniforms one through singular maps, other new phenomena and pathologies will appear. In the case of $1-d$ waves in continuous heterogeneous media, the lack of $C^{1}$-regularity of the coefficients allows exhibiting unexpected concentration phenomena of the high frequency waves that contradict all the well-known propagation and dispersion properties of waves in homogeneous media (see [5]). Systematic analysis of this extra possible pathologies related to the irregularity of the numerical meshes is to be developed. We refer the interested reader to the book by Cohen [6] where a careful analysis of transmission-reflection phenomena is carried on in the case of two uniform grids with different meshes sizes.

2. Other numerical schemes. In this paper we have considered the case of finite difference approximations. It would be interesting to develop the symbolic calculus under consideration for other numerical schemes such as finite elements, mixed or discontinuous Galerkin finite elements. This will be done in a forthcoming paper. In particular, for the mixed finite elements in $[9$ we expect the corresponding principal symbol to be $p(x, t, \xi, \tau)=g^{\prime}(x) \varrho(g(x)) \tau^{2}-4 \tan ^{2}(\xi / 2) \sigma(g(x)) / g^{\prime}(x)$ for which the corresponding Hamiltonian system is $x^{\prime}(t)=c_{g}(x(t)) / \cos ^{2}(\xi(t) / 2)$ and $\xi^{\prime}(t)=-2 c_{g}^{\prime}(x(t)) \tan (\xi(t) / 2)$, where $c_{g}(x):=\sqrt{\sigma / \varrho}(g(x)) / g^{\prime}(x)$. The good observability features of the mixed finite element scheme proved in [9] could be explained by the absence of fixed points for the above Hamiltonian system since $x^{\prime}(t)$ cannot vanish.

3. Multi-dimensional waves. The extension of the analysis in this paper to the multi-dimensional case is a challenging problem. Our techniques can be employed to deal with non-uniform meshes obtained as diffeomorphic transformations of a uniform grid, for instance in the context of finite differences. But, of course, in the finite element element setting, it is common to use and deal with meshes that are not topologically equivalent to an uniform one. Adapting the our analysis to that framework requires of significant further developments.

4. Filtering mechanisms on non-uniform meshes. Our analysis in this paper shows the necessity of using filtering mechanisms on non-uniform regular meshes whose corresponding Hamiltonian systems have fixed points. In [25, we proved the efficiency of the numerical viscosity method in the context of the boundary stabilization for the variable coefficients wave equation approximated by three-points finite difference schemes on regular non-uniform meshes. However, for the best of our knowledge, nothing is known concerning other well-known filtering techniques on uniform media like the Fourier truncation and the bi-grid techniques [1].

\section{Appendix A. Proof of some technical Results}

A.1. Proof of Theorem 2.1. We deduce only equation 2.12 for the limit measure $\mathcal{W}(y, t, \xi)$.

Step I. Weak convergence of the Wigner transforms $\mathcal{W}^{\epsilon}\left[w^{\epsilon}\right]$. In order to simplify the notation, in this proof we skip the argument $\left[w^{\epsilon}\right]$ accompanying the Wigner transform $\mathcal{W}^{\epsilon}\left[w^{\epsilon}\right](y, t, \xi)$ to write $\mathcal{W}^{\epsilon}(y, t, \xi)$. Using the boundedness of $\left(w^{\epsilon, 0}\right)_{\epsilon}$ in $L^{2}(\mathbb{R})$ as $\epsilon \rightarrow 0$ and the conservation in time of the $L^{2}$-norm of $w^{\epsilon}(\cdot, t)$, it can be proved that the corresponding Wigner transform $\mathcal{W}^{\epsilon}(y, t, \xi)$ is bounded in $\mathcal{S}^{\prime}\left(\mathbb{R}_{y} \times \mathbb{R}_{\xi}\right)$ for all $t \geq 0$. Modulo extracting subsequences, we have

$$
\mathcal{W}^{\epsilon}(y, t, \xi) \rightarrow \mathcal{W}(y, t, \xi) \text { weakly star in } \mathcal{S}^{\prime}\left(\mathbb{R}_{y} \times \mathbb{R}_{\xi}\right) \text { as } \epsilon \rightarrow 0, \forall t \geq 0,
$$


where $\mathcal{W}$ is a positive Radon measure.

Step II. Write the equation satisfied by the Wigner transform $\mathcal{W}^{\epsilon}$. Using the equation 2.8 for $w^{\epsilon}(y, t)$, it is easy to see that the Fourier transform of $\mathcal{W}^{\epsilon}$ in $\xi, \widehat{\mathcal{W}}^{\epsilon}(y, t, z)$, satisfies the following equation:

$$
\partial_{t} \widehat{\mathcal{W}}^{\epsilon}(y, t, x)=-\left[\mathcal{K}_{c}^{\epsilon,+}(y, z) \partial_{y} \widehat{\mathcal{W}}^{\epsilon}(y, t, z)+\mathcal{K}_{c}^{\epsilon,-}(y, z) \partial_{z} \widehat{\mathcal{W}}^{\epsilon}(y, t, z)+2 \mathcal{K}_{d}^{\epsilon,+}(y, z) \widehat{\mathcal{W}}^{\epsilon}(y, t, z)\right]
$$

where

$$
\mathcal{K}_{1, c}^{\epsilon,+}(y, z):=\frac{1}{2}\left(c\left(y+\frac{\epsilon z}{2}\right)+c\left(y-\frac{\epsilon z}{2}\right)\right) \text { and } \mathcal{K}_{c}^{\epsilon,-}(y, z):=\frac{1}{\epsilon}\left(c\left(y+\frac{\epsilon z}{2}\right)-c\left(y-\frac{\epsilon z}{2}\right)\right) .
$$

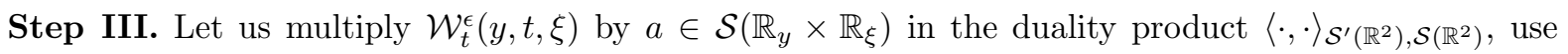
Parseval identity in $\xi$ and A.2 to obtain:

$$
\begin{aligned}
& \left\langle\partial_{t} \mathcal{W}^{\epsilon}, a\right\rangle_{\mathcal{S}^{\prime}\left(\mathbb{R}^{2}\right), \mathcal{S}\left(\mathbb{R}^{2}\right)}=\frac{1}{2 \pi}\left\langle\partial_{t} \widehat{\mathcal{W}^{\epsilon}}, \overline{\bar{a}}\right\rangle_{\mathcal{S}^{\prime}\left(\mathbb{R}^{2}\right), \mathcal{S}\left(\mathbb{R}^{2}\right)}=-\left(\mathcal{I}_{1}^{\epsilon}(t)+\mathcal{I}_{2}^{\epsilon}(t)+\mathcal{I}_{3}^{\epsilon}(t)\right):= \\
& =-\left(\frac{1}{2 \pi}\left\langle\mathcal{K}_{c}^{\epsilon,+} \partial_{y} \widehat{\mathcal{W}}^{\epsilon}, \overline{\bar{a}}\right\rangle_{\mathcal{S}^{\prime}\left(\mathbb{R}^{2}\right), \mathcal{S}\left(\mathbb{R}^{2}\right)}+\frac{1}{2 \pi}\left\langle\mathcal{K}_{c}^{\epsilon,-} \partial_{z} \widehat{\mathcal{W}}^{\epsilon}, \overline{\bar{a}}\right\rangle_{\mathcal{S}^{\prime}\left(\mathbb{R}^{2}\right), \mathcal{S}\left(\mathbb{R}^{2}\right)}+\frac{1}{2 \pi}\left\langle 2 \mathcal{K}_{d}^{\epsilon,+} \widehat{\mathcal{W}^{\epsilon}}, \overline{\bar{a}}\right\rangle_{\left.\mathcal{S}^{\prime}\left(\mathbb{R}^{2}\right), \mathcal{S}\left(\mathbb{R}^{2}\right)\right)}\right) .
\end{aligned}
$$

The terms $\mathcal{I}_{1}^{\epsilon}(t)$ and $\mathcal{I}_{2}^{\epsilon}(t)$ are generated by the principal operator in $(2.8), c(y) \partial_{y}$, and $\mathcal{I}_{3}^{\epsilon}(t)$ is the contribution of the potential $d(y) w^{\epsilon}$. Let us pass to the limit in each term $\overline{\mathcal{I}}_{j}^{\epsilon}(t), 1 \leq j \leq 3$. By passing the derivatives in $y$ and $z$ from $\mathcal{W}^{\epsilon}$ to the other factors, taking into account that $\partial_{y} \mathcal{K}_{c}^{\epsilon,+}=\mathcal{K}_{c^{\prime}}^{\epsilon,+}$ and $\partial_{z} \mathcal{K}_{c}^{\epsilon,-}=\mathcal{K}_{c^{\prime}}^{\epsilon,+}$ and applying once more the Parseval identity in $z$, we have

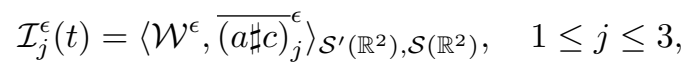

where

$$
\begin{aligned}
& (a \sharp c)_{1}^{\epsilon}(y, \xi)=-\frac{1}{2 \pi} \int_{\mathbb{R}}\left(\mathcal{K}_{c^{\prime}}^{\epsilon,+}(y, z) \widehat{\bar{a}}(y, z)+\mathcal{K}_{c}^{\epsilon,+}(y, z) \partial_{y} \widehat{\bar{a}}(y, z)\right) \exp (i \xi z) d z, \\
& (a \sharp c)_{2}^{\epsilon}(y, \xi)=-\frac{1}{2 \pi} \int_{\mathbb{R}}\left(\mathcal{K}_{c^{\prime}}^{\epsilon,+}(y, z) \widehat{\bar{a}}(y, z)+\mathcal{K}_{c}^{\epsilon,-}(y, z) \partial_{z} \widehat{\bar{a}}(y, z)\right) \exp (i \xi z) d z
\end{aligned}
$$

and

$$
(a \sharp c)_{3}^{\epsilon}(y, \xi)=\frac{1}{2 \pi} \int_{\mathbb{R}} 2 \mathcal{K}_{d}^{\epsilon,+}(y, z) \widehat{\bar{a}}(y, z) \exp (i \xi z) d z .
$$

Let us remark that, by Taylor expansions of $\tilde{c}(y \pm \epsilon z / 2)$ around $y$, for all regular functions $\tilde{c}$, we get

$$
\mathcal{K}_{\tilde{c}}^{\epsilon,+}(y, z):=\frac{1}{2}\left(\tilde{c}\left(y+\frac{\epsilon z}{2}\right)+\tilde{c}\left(y-\frac{\epsilon z}{2}\right)\right) \sim \tilde{c}(y) \text { and } \mathcal{K}_{\tilde{c}}^{\epsilon,-}(y, z):=\frac{1}{\epsilon}\left(\tilde{c}\left(y+\frac{\epsilon z}{2}\right)-\tilde{c}\left(y-\frac{\epsilon z}{2}\right)\right) \sim \tilde{c}^{\prime}(y) z
$$

as $\epsilon \rightarrow 0\left(C^{1}(\mathbb{R})\right.$ and $C^{2}(\mathbb{R})$ in the first/second case), so that $(a \sharp c)_{j}^{\epsilon}(y, \xi) \rightarrow(a \sharp c)_{j}(y, \xi)$ in $\mathcal{S}\left(\mathbb{R}^{2}\right)$, where

$$
(a \sharp c)_{1}(y, \xi):=-c^{\prime}(y) \bar{a}(y, \xi)-c(y) \partial_{y} \bar{a}(y, \xi), \quad(a \sharp c)_{2}(y, \xi):=-c^{\prime}(y) \bar{a}(y, \xi)+c^{\prime}(y) \partial_{\xi}(\xi \bar{a}(y, \xi)) .
$$

and

$$
(a \sharp c)_{3}(y, \xi):=2 d(y) \bar{a}(y, \xi) .
$$

Passing the derivatives of $a$ with respect to $y$ or $\xi$ in the right hand side of $(\mathrm{A} .6)$ to $\mathcal{W}$ in $(\mathrm{A} .4$ and taking

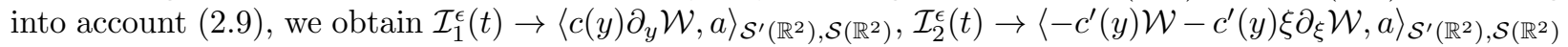

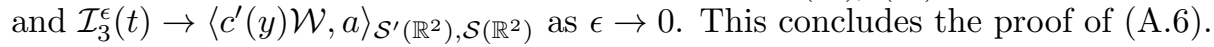


A.2. Proof of Theorem 2.2. The first proof follows the same steps as the one of Theorem 2.1 and we start directly with Step II which has some differences with respect to the corresponding one in the proof of Theorem 2.1. Let us remark that the Fourier transforms of $\mathcal{W}^{\epsilon, \pm}(y, t, \xi)$ in $\xi, \widehat{\mathcal{W}}^{\epsilon, \pm}(y, t, z)$, satisfy the system:

$$
\begin{aligned}
\partial_{t} \widehat{\mathcal{W}}^{\epsilon, \pm} & = \pm \mathcal{K}_{c}^{\epsilon,+}(y, z) \partial_{y} \widehat{\mathcal{W}}^{\epsilon, \pm} \pm \mathcal{K}_{c}^{\epsilon,-}(y, z) \partial_{z} \widehat{\mathcal{W}}^{\epsilon, \pm} \pm \mathcal{K}_{c^{\prime}}^{\epsilon,+}(y, z) \widehat{\mathcal{W}}^{\epsilon, \pm} \\
& \mp \frac{1}{2} \mathcal{K}_{\widetilde{c}}^{\epsilon,+}(y, z) \widehat{\widetilde{\mathcal{W}}}{ }^{\epsilon,+} \mp \frac{\epsilon}{4} \mathcal{K}_{\widetilde{c}}^{\epsilon,-}(y, z) \widehat{\tilde{\mathcal{W}}}{ }^{\epsilon,-} .
\end{aligned}
$$

Here, $K_{c}^{\epsilon, \pm}$ are as in A.2. Observe that the first line in A.7) is precisely of the same type as A.2 in which the the arguments in Step III in the proof of Theorem 2.1 suffice to pass to the limit. The only remaining thing is to pass to the limit in the second line of A.7 which does not depend on $\widehat{\mathcal{W}}^{\epsilon, \pm}$, but on the Fourier transforms of $\tilde{\mathcal{W}}^{\epsilon, \pm}$ in 2.18) satisfying the system:

$$
\partial_{t} \widehat{\widehat{\mathcal{W}}}^{\epsilon, \pm}=-\frac{2}{\epsilon} \mathcal{K}_{c}^{\epsilon,+}(y, z) \partial_{z} \widehat{\widetilde{\mathcal{W}}}^{\epsilon, \mp}-\frac{\epsilon}{2} \mathcal{K}_{c}^{\epsilon,-}(y, z) \partial_{y} \widehat{\widetilde{\mathcal{W}}}^{\epsilon, \mp}-\frac{\epsilon}{2} \mathcal{K}_{c^{\prime}}^{\epsilon,-}(y, z) \widehat{\hat{\mathcal{W}}}^{\epsilon, \mp}+\widehat{A}^{\epsilon, \pm}
$$

where

$$
\widehat{A}^{\epsilon,+}:=\mathcal{K}_{\tilde{c}}^{\epsilon,+}(y, z)\left(\widehat{\mathcal{W}}^{\epsilon,+}-\widehat{\mathcal{W}}^{\epsilon,-}\right) \text { and } \widehat{A}^{\epsilon,-}:=\frac{\epsilon}{2} \mathcal{K}_{\tilde{c}}^{\epsilon,-}(y, z)\left(\widehat{\mathcal{W}}^{\epsilon,+}+\widehat{\mathcal{W}}^{\epsilon,-}\right) .
$$

Remark that both $\tilde{\mathcal{W}}^{\epsilon, \pm}$ are bounded as $\epsilon \rightarrow 0$ in $\mathcal{S}^{\prime}\left(\mathbb{R}_{y} \times \mathbb{R}_{\xi}\right)$, so that, modulo extracting subsequences, they converge weakly star to $\tilde{\mathcal{W}}^{ \pm}$in $\mathcal{S}^{\prime}\left(\mathbb{R}^{2}\right)$. The second term of the second line in A.7 is of order $\epsilon$, so that it converge to zero as $\epsilon \rightarrow 0$. The first term is of order one and, following the same arguments in Step III of the proof of Theorem 2.1. we can prove that for all $a \in \mathcal{S}\left(\mathbb{R}^{2}\right)$ the following convergence holds as $\epsilon \rightarrow 0$ :

$$
\mp \frac{1}{2} \frac{1}{2 \pi}\left\langle\mathcal{K}_{\tilde{c}}^{\epsilon,+} \widehat{\tilde{\mathcal{W}}}^{\epsilon,+}, \overline{\bar{a}}_{\mathcal{S}^{\prime}\left(\mathbb{R}^{2}\right), \mathcal{S}\left(\mathbb{R}^{2}\right)} \rightarrow \mp \frac{1}{2}\left\langle\tilde{c}(y) \tilde{\mathcal{W}}^{+}, a\right\rangle_{\mathcal{S}^{\prime}\left(\mathbb{R}^{2}\right), \mathcal{S}\left(\mathbb{R}^{2}\right)} .\right.
$$

In order to get 2.20 , we have to prove that, for all $\xi \neq 0, \tilde{\mathcal{W}}^{+}(y, t, \xi)=0$. This is obtained by passing to the limit in A.8). Let us observe that the first term in the right hand side of $(\mathrm{A} .8)$ is of order $\epsilon^{-1}$, the following two are of order $\epsilon$, while the fourth one and the time derivative on the left hand side are of order one. By multiplying $($ A.8 $)$ by $\epsilon \overline{\bar{a}}(x, z) / 2 \pi\left(a \in \mathcal{S}\left(\mathbb{R}^{2}\right)\right)$ in the duality product $\langle\cdot, \cdot\rangle_{\mathcal{S}^{\prime}\left(\mathbb{R}^{2}\right), \mathcal{S}\left(\mathbb{R}^{2}\right)}$ and passing to the limit as $\epsilon \rightarrow 0$, the duality products corresponding to all terms in A.8 converge to zero, excepting for the one corresponding to the first term on the right hand side. Thus,

$$
\lim _{\epsilon \rightarrow 0} \frac{1}{2 \pi}\left\langle\mathcal{K}_{c}^{\epsilon,+} \partial_{z} \widehat{\mathcal{W}}^{\epsilon, \pm}, \overline{\bar{a}}\right\rangle_{\mathcal{S}^{\prime}\left(\mathbb{R}^{2}\right), \mathcal{S}\left(\mathbb{R}^{2}\right)}=\left\langle c(y)(-i \xi) \tilde{\mathcal{W}}^{ \pm}, a\right\rangle_{\mathcal{S}^{\prime}\left(\mathbb{R}^{2}\right), \mathcal{S}\left(\mathbb{R}^{2}\right)}=0 .
$$

Since $c \neq 0$, we obtain that, for all $\xi \neq 0, \tilde{\mathcal{W}}^{ \pm}=0$. This concludes 2.20 .

Set $\mathbf{w}(y, t):=(w(y, t), \tilde{w}(y, t))$ to be the column vector containing the two unknowns in (2.14) and $\boldsymbol{\Theta}(y, \xi):=\boldsymbol{\Theta}_{1}(y, \xi)+\epsilon \boldsymbol{\Theta}_{0}(y, \xi)$, where

$$
\boldsymbol{\Theta}_{1}(y, \xi):=\left(\begin{array}{cc}
0 & c(y) i \xi \\
c(y) i \xi & 0
\end{array}\right) \text { and } \boldsymbol{\Theta}_{0}(y, \xi):=\left(\begin{array}{cc}
0 & d(y) \\
e(y) & 0
\end{array}\right) .
$$

Thus, using the notation in Subsection 2.2, system 2.14 can be written in its pseudo-differential form as

$$
\partial_{t} \mathbf{w}(y, t)=\frac{1}{\epsilon} \boldsymbol{\Theta}\left(y, \epsilon \partial_{y}\right) \mathbf{w}(y, t)=\left(\frac{1}{\epsilon} \boldsymbol{\Theta}_{1}\left(y, \epsilon \partial_{y}\right)+\boldsymbol{\Theta}_{0}\left(y, \epsilon \partial_{y}\right)\right) \mathbf{w}(y, t) .
$$

The matrix $\boldsymbol{\Theta}_{1}(y, \xi)$ admits the Fourier decomposition $\boldsymbol{\Theta}_{1}(y, \xi)=i \boldsymbol{\Delta} \boldsymbol{\Lambda}(y, \xi) \boldsymbol{\Delta}^{*}$, where

$$
\boldsymbol{\Lambda}(y, \xi):=\left(\begin{array}{cc}
\lambda_{c, \xi}^{+}(y, \xi) & 0 \\
0 & \lambda_{c, \xi}^{-}(y, \xi)
\end{array}\right), \quad \boldsymbol{\Delta}:=\frac{1}{\sqrt{2}}\left(\begin{array}{cc}
1 & 1 \\
1 & -1
\end{array}\right),
$$

and $\lambda_{c, \xi}^{ \pm}(y, \xi):= \pm c(y) \xi$. Remark that the eigenvector matrix $\boldsymbol{\Delta}$ in A.11 does not depend on anyone of the variables $y$ and $\xi$. Let us define the projectors $\boldsymbol{\Delta}^{ \pm}$to be

$$
\boldsymbol{\Delta}^{+}:=\boldsymbol{\Delta}\left(\begin{array}{ll}
1 & 0 \\
0 & 0
\end{array}\right) \boldsymbol{\Delta}^{*}=\frac{1}{2}\left(\begin{array}{ll}
1 & 1 \\
1 & 1
\end{array}\right) \text { and } \boldsymbol{\Delta}^{-}=\boldsymbol{\Delta}\left(\begin{array}{ll}
0 & 0 \\
0 & 1
\end{array}\right) \boldsymbol{\Delta}^{*}=\frac{1}{2}\left(\begin{array}{cc}
1 & -1 \\
-1 & 1
\end{array}\right) \text {. }
$$


Set $\omega(\xi):=\xi$. Remark that he matrix $\boldsymbol{\Theta}_{1}(y, \xi)$ associated to the principal operator in A.10 can be decomposed as

$$
\boldsymbol{\Theta}_{1}=i \lambda_{c, \omega}^{+} \boldsymbol{\Delta}^{+}+i \lambda_{c, \omega}^{-} \boldsymbol{\Delta}^{-}
$$

Moreover, the following identities hold $\left(\mathbf{0}_{d}, \mathbf{I}_{d}\right.$ being the $d$-dimensional null and identity matrix $)$ :

$$
\boldsymbol{\Delta}^{ \pm} \boldsymbol{\Delta}^{ \pm}=\boldsymbol{\Delta}^{ \pm}, \quad \boldsymbol{\Delta}^{ \pm} \boldsymbol{\Delta}^{\mp}=\mathbf{0}_{2}, \quad \boldsymbol{\Delta}^{+}+\boldsymbol{\Delta}^{-}=\mathbf{I}_{2}
$$

In what follows, we give a second proof of Theorem 2.2 which follows the one of Theorem 6.1 in [15], is more technical than the first proof we gave, but provides additional information with respect to that one. It highlights the interpretation of the results in Theorem 2.2 in terms of the spectral decomposition of $\boldsymbol{\Theta}_{1}(y, \xi)$.

Consider initial data $\mathbf{w}^{\epsilon, 0}=\left(w^{\epsilon, 0}, \tilde{w}^{\epsilon, 0}\right)$ in 2.14 depending on a small parameter $\epsilon$ and denote $\mathbf{w}^{\epsilon}(y, t)$ the corresponding solution. For simplicity, set $\mathcal{W}^{\epsilon}:=\mathcal{W}^{\epsilon}\left[\mathbf{w}^{\epsilon}\right]$ to be the Wigner transform matrix of $\mathbf{w}^{\epsilon}$.

Step I. Equation (A.19) of the Wigner transform matrix $\mathcal{W}^{\epsilon}$. Using the definition 2.60 of the Wigner transform and equation A.10, we obtain the following expression of the Fourier transform in $\xi$ of $\mathcal{W}^{\epsilon}(y, t, \xi), \widehat{\mathcal{W}}^{\epsilon}(y, t, z)$ :

$$
\begin{aligned}
& \partial_{t} \widehat{\mathcal{W}}^{\epsilon}(y, t, z)= \\
& =\frac{1}{\epsilon} \boldsymbol{\Theta}\left(y, \epsilon \partial_{y}\right) \mathbf{w}^{\epsilon}\left(y-\frac{\epsilon z}{2}, t\right) \otimes\left(\mathbf{w}^{\epsilon}\left(y+\frac{\epsilon z}{2}, t\right)\right)^{*}+\mathbf{w}^{\epsilon}\left(y-\frac{\epsilon z}{2}, t\right) \otimes \frac{1}{\epsilon}\left(\boldsymbol{\Theta}\left(y, \epsilon \partial_{y}\right) \mathbf{w}^{\epsilon}\left(y+\frac{\epsilon z}{2}, t\right)\right)^{*} .
\end{aligned}
$$

Let us consider $a \in \mathcal{S}\left(\mathbb{R}_{y} \times \mathbb{R}_{\xi}\right)$ and multiply A.14 by $\overline{\bar{a}}(y, z) / 2 \pi$. After writing explicitly how the pseudo-differential operator $\boldsymbol{\Theta}\left(y, \epsilon \partial_{y}\right)$ acts in A.14, we obtain:

$$
\frac{1}{2 \pi}\left\langle\partial_{t} \widehat{\mathcal{W}}^{\epsilon}, \overline{\bar{a}}_{\mathcal{S}^{\prime}\left(\mathbb{R}^{2}\right), \mathcal{S}\left(\mathbb{R}^{2}\right)}=\mathbf{A}^{\epsilon}+\mathbf{B}^{\epsilon}\right.
$$

where

$$
\mathbf{A}^{\epsilon}:=\frac{1}{(2 \pi)^{2}} \int_{\mathbb{R}^{4}} \frac{1}{\epsilon} \boldsymbol{\Theta}\left(y-\frac{\epsilon z}{2}, \epsilon \eta\right) \mathbf{w}^{\epsilon}(x, t) \otimes\left(\mathbf{w}^{\epsilon}\left(y+\frac{\epsilon z}{2}, t\right)\right)^{*} \exp \left(i \eta\left(y-\frac{\epsilon z}{2}-x\right)\right) \overline{\bar{a}}(y, z) d x d y d z d \eta
$$

and

$\mathbf{B}^{\epsilon}:=\frac{1}{(2 \pi)^{2}} \int_{\mathbb{R}^{4}} \mathbf{w}^{\epsilon}\left(y-\frac{\epsilon z}{2}, t\right) \otimes\left(\mathbf{w}^{\epsilon}(x, t)\right)^{*}\left(\frac{1}{\epsilon} \boldsymbol{\Theta}\left(y+\frac{\epsilon z}{2}, \epsilon \eta\right)\right)^{*} \exp \left(-i \eta\left(y+\frac{\epsilon z}{2}-x\right)\right) \overline{\bar{a}}(y, z) d x d y d z d \eta$.

In $\mathbf{A}^{\epsilon}$ and $\mathbf{B}^{\epsilon}$ consider the change of variable $\zeta:=\epsilon \eta$ (thus, $d \eta:=\epsilon^{-1} d \zeta$ ). We also do the change of variable $y \rightarrow y^{\prime}$ so that $y+\epsilon z / 2=2 y^{\prime}-x$ (in $\mathbf{A}^{\epsilon}$ ) and $y-\epsilon z / 2=2 y^{\prime}-x$ (in $\mathbf{B}^{\epsilon}$ ) (thus, $d y=2 d y^{\prime}$ ). Moreover, in $\mathbf{B}^{\epsilon}$ we change $x \rightarrow x^{\prime}=2 y^{\prime}-x$. After all these changes, $\mathbf{A}^{\epsilon}$ and $\mathbf{B}^{\epsilon}$ become

$\mathbf{A}^{\epsilon}=\frac{1}{(2 \pi)^{2}} \frac{2}{\epsilon} \int_{\mathbb{R}^{4}} \frac{1}{\epsilon} \boldsymbol{\Theta}(2 y-x-\epsilon z, \zeta) \mathbf{w}^{\epsilon}(x, t) \otimes\left(\mathbf{w}^{\epsilon}(2 y-x, t)\right)^{*} \exp \left(\frac{2 i \zeta}{\epsilon}\left(y-x-\frac{\epsilon z}{2}\right)\right) \overline{\bar{a}}\left(2 y-x-\frac{\epsilon z}{2}, z\right) d x d y d z d \zeta$ and

$\mathbf{B}^{\epsilon}=\frac{1}{(2 \pi)^{2}} \frac{2}{\epsilon} \int_{\mathbb{R}^{4}} \mathbf{w}^{\epsilon}(x, t) \otimes\left(\mathbf{w}^{\epsilon}(2 y-x, t)\right)^{*}\left(\frac{1}{\epsilon} \boldsymbol{\Theta}(x+\epsilon z, \zeta)\right)^{*} \exp \left(\frac{2 i \zeta}{\epsilon}\left(y-x-\frac{\epsilon z}{2}\right)\right) \overline{\bar{a}}\left(x+\frac{\epsilon z}{2}, z\right) d x d y d z d \zeta$.

Remark that $2 y-x-\epsilon z=y+y_{1}, x+\epsilon z=y-y_{1}, 2 y-x-\epsilon z / 2=y+y_{2}$ and $x+\epsilon z / 2=y-y_{2}$, with $y_{1}=y-x-\epsilon z$ and $y_{2}=y-x-\epsilon z / 2$. The following Taylor expansions of $\boldsymbol{\Theta}\left(y \pm y_{1}, \zeta\right)$ and $\overline{\bar{a}}\left(y \pm y_{2}, z\right)$ about $y$ hold:

$$
\boldsymbol{\Theta}\left(y \pm y_{1}, \zeta\right)=\boldsymbol{\Theta}(y, \zeta) \pm y_{1} \partial_{y} \boldsymbol{\Theta}(y, \zeta)+y_{1}^{2} \boldsymbol{\mathcal { R }}_{\Theta}^{ \pm} \text {and } \overline{\bar{a}}\left(y \pm y_{2}, z\right)=\overline{\bar{a}}(y, z) \pm y_{2} \partial_{y} \overline{\bar{a}}(y, z)+y_{2}^{2} \mathcal{R}_{a}^{ \pm}
$$

where $\mathcal{R}_{\Theta}^{ \pm}=\mathcal{R}_{\Theta}^{ \pm}\left(y, y_{1}, \zeta\right)$ and $\mathcal{R}_{a}^{ \pm}=\mathcal{R}_{a}^{ \pm}\left(y, y_{2}, z\right)$ are the corresponding Taylor remainders. We write $\mathbf{A}^{\epsilon}$ and $\mathbf{B}^{\epsilon}$ as $\mathbf{A}^{\epsilon}:=\tilde{\mathbf{A}}^{\epsilon}+\mathcal{R}_{\mathbf{A}}^{\epsilon}$ and $\mathbf{B}^{\epsilon}:=\tilde{\mathbf{B}}^{\epsilon}+\mathcal{R}_{\mathbf{B}}^{\epsilon}$, where $\tilde{\mathbf{A}}^{\epsilon}$ and $\tilde{\mathbf{B}}^{\epsilon}$ are integrals of the same form as $\mathbf{A}^{\epsilon}$ and $\mathbf{B}^{\epsilon}$ retaining from the factors $\boldsymbol{\Theta}\left(y \pm y_{1}, \zeta\right) \overline{\bar{a}}\left(y \pm y_{2}, z\right)$ appearing in $\mathbf{A}^{\epsilon}$ and $\mathbf{B}^{\epsilon}$ only the terms $\boldsymbol{\Theta}(y, \zeta) \overline{\bar{a}}(y, z) \pm y_{2} \boldsymbol{\Theta}(y, \zeta) \partial_{y} \overline{\bar{a}}(y, z) \pm y_{1} \partial_{y} \boldsymbol{\Theta}(y, \zeta) \overline{\bar{a}}(y, z)$. We follow by using the following equivalent form of 
the Wigner transform matrix 2.6 which can be obtained by the change of variable $z \rightarrow y=x-\epsilon z / 2$ in 2.6.

$$
\mathcal{W}^{\epsilon}\left[\mathbf{f}^{1}, \mathbf{f}^{2}\right](x, \xi):=\frac{1}{2 \pi} \frac{2}{\epsilon} \int_{\mathbb{R}} \mathbf{f}^{1}(y) \otimes \mathbf{f}^{2, *}(2 x-y) \exp \left(\frac{2 i \xi(x-y)}{\epsilon}\right) d y
$$

We also take into account that the term $y-x$ entering in both $y_{1}$ and $y_{2}$ yields $\epsilon \partial_{\zeta} \mathcal{W}^{\epsilon}(y, t, \zeta) / 2 i$ when integrate in $x$ in both $\tilde{\mathbf{A}}^{\epsilon}$ and $\tilde{\mathbf{B}}^{\epsilon}$, while the term $-\epsilon z \partial_{y}^{\alpha} \overline{\bar{a}}(y, z)$ yields $\epsilon \partial_{\zeta} \partial_{y}^{\alpha} a(y, \zeta) / i$ when integrate in $z$ (with $\alpha=0$ or $\alpha=1$ ). Thus,

$$
\begin{aligned}
\tilde{\mathbf{A}}^{\epsilon} & :=\frac{1}{\epsilon}\left\langle\boldsymbol{\Theta} \mathcal{W}^{\epsilon}(\cdot, t, \cdot), a\right\rangle_{\mathcal{S}^{\prime}, \mathcal{S}}+\frac{1}{2 i}\left\langle\boldsymbol{\Theta} \partial_{\xi} \mathcal{W}^{\epsilon}(\cdot, t, \cdot), \partial_{y} a\right\rangle_{\mathcal{S}^{\prime}, \mathcal{S}}+\frac{1}{2 i}\left\langle\boldsymbol{\Theta} \mathcal{W}^{\epsilon}(\cdot, t, \cdot), \partial_{y \xi}^{2} a\right\rangle_{\mathcal{S}^{\prime}, \mathcal{S}} \\
& +\frac{1}{2 i}\left\langle\partial_{y} \boldsymbol{\Theta} \partial_{\xi} \mathcal{W}^{\epsilon}(\cdot, t, \cdot), a\right\rangle_{\mathcal{S}^{\prime}, \mathcal{S}}+\frac{1}{i}\left\langle\partial_{y} \boldsymbol{\Theta} \mathcal{W}^{\epsilon}(\cdot, t, \cdot), \partial_{\xi} a\right\rangle_{\mathcal{S}^{\prime}, \mathcal{S}}
\end{aligned}
$$

and a similar expression for $\tilde{\mathbf{B}}^{\epsilon}$, in which the only changes are that the terms in the integrand from the second to the fifth ones change the sign, the matrix $\boldsymbol{\Theta}(y, \xi)$ and its derivatives are replaced by the corresponding conjugate transpose matrices and interchanged with the corresponding derivatives of the Wigner transform matrix $\mathcal{W}^{\epsilon}(y, t, \xi)$. Here, $\langle f, a\rangle_{\mathcal{S}^{\prime}, \mathcal{S}}:=\int_{\mathbb{R}^{2}} f(y, \xi) a(y, \xi) d y d \xi$. Passing all the derivatives of $a$ with respect to both $y$ and $\xi$ to the accompanying factors, we obtain

$$
\tilde{\mathbf{A}}^{\epsilon}=\left\langle\frac{1}{\epsilon} \boldsymbol{\Theta} \mathcal{W}^{\epsilon}(\cdot, t, \cdot)+\frac{1}{2 i}\left\{\boldsymbol{\Theta}, \mathcal{W}^{\epsilon}(\cdot, t, \cdot)\right\}-\frac{1}{2 i} \partial_{y \xi}^{2} \boldsymbol{\Theta} \mathcal{W}^{\epsilon}(\cdot, t, \cdot), a\right\rangle_{\mathcal{S}^{\prime}, \mathcal{S}}
$$

and a similar expression for $\tilde{\mathbf{B}}^{\epsilon}$, in which the sign of the third term is changed and the above mentioned transformations concerning the conjugate transposition of $\boldsymbol{\Theta}(y, \xi)$ and the interchange with $\mathcal{W}^{\epsilon}(y, t, \xi)$ are done. Here, $\{p, q\}:=\partial_{\xi} p \partial_{y} q-\partial_{y} p \partial_{\xi} q$ is the so-called Poisson bracket (cf. [15]).

Let us show that the terms $\boldsymbol{\mathcal { R }}_{\mathrm{A}}^{\epsilon}$ and $\boldsymbol{\mathcal { R }}_{\mathrm{B}}^{\epsilon}$ are small with respect to $\epsilon$. We explain how this can be proved for $\mathcal{R}_{\mathbf{A}}^{\epsilon}$, for $\mathcal{R}_{\mathbf{B}}^{\epsilon}$ the arguments being similar. Remark that $\boldsymbol{\mathcal { R }}_{\mathbf{A}}^{\epsilon}$ is an integral of the same type as $\mathbf{A}^{\epsilon}$, in which the factor $\boldsymbol{\Theta}\left(y+y_{1}, \zeta\right) \overline{\bar{a}}\left(y+y_{2}, z\right)$ is replaced by $y_{2}^{2} \boldsymbol{\Theta}(y, \zeta) \mathcal{R}_{a}^{+}+y_{1} y_{2} \partial_{y} \boldsymbol{\Theta}(y, \zeta) \partial_{y} \overline{\bar{a}}(y, z)+y_{1} y_{2}^{2} \partial_{y} \boldsymbol{\Theta}(y, \zeta) \mathcal{R}_{a}^{+}+$ $y_{1}^{2} \boldsymbol{\mathcal { R }}_{\boldsymbol{\Theta}}^{+} \overline{\bar{a}}(y, z)+y_{1}^{2} y_{2} \boldsymbol{\mathcal { R }}_{\boldsymbol{\Theta}}^{+} \partial_{y} \overline{\bar{a}}(y, z)+y_{1}^{2} y_{2}^{2} \boldsymbol{\mathcal { R }}_{\boldsymbol{\Theta}}^{+} \mathcal{R}_{a}^{+}$. Each term of this integrand contains powers of the form $y_{1}^{\alpha} y_{2}^{\beta}$, with $\alpha+\beta \geq 2$. In order to make clear the ideas, we analyze only the integral $\mathcal{I}^{\epsilon}$ involving the term $y_{1} y_{2} \partial_{y} \boldsymbol{\Theta}(y, \zeta) \partial_{y} \overline{\bar{a}}(y, z)$. For the other ones, one can do a similar analysis, taking into account the fact that $\boldsymbol{\mathcal { R }}_{\boldsymbol{\Theta}}^{+} \sim \partial_{y}^{2} \boldsymbol{\Theta}(y, \zeta) / 2$ and $\mathcal{R}_{a}^{+} \sim \partial_{y}^{2} \overline{\bar{a}}(y, z) / 2$. Observe that $y_{1} y_{2}=(y-x)^{2}-3 \epsilon z(y-x) / 2+\epsilon^{2} z^{2} / 2$. As before, we take into account the fact that $(y-x)^{\alpha}$ yields $(\epsilon / 2 i)^{\alpha} \partial_{\zeta}^{\alpha} \mathcal{W}^{\epsilon}(y, t, \zeta)$ when integrate in $x$, while any factor $(\epsilon z)^{\alpha} \partial_{y}^{\beta} \overline{\bar{a}}(y, z)$ is converted into $(-\epsilon / i)^{\alpha} \partial_{\zeta}^{\alpha} \partial_{y}^{\beta} a(y, \zeta)$ when integrate in $z$. In this way,

$$
\mathcal{I}^{\epsilon}=-\frac{\epsilon}{4}\left\langle\frac{1}{4} \partial_{y} \boldsymbol{\Theta} \partial_{\xi}^{2} \mathcal{W}^{\epsilon}(\cdot, t, \cdot), \partial_{y} a\right\rangle_{\mathcal{S}^{\prime}, \mathcal{S}}-\frac{\epsilon}{2}\left\langle\partial_{y} \boldsymbol{\Theta} \partial_{\xi} \mathcal{W}^{\epsilon}(\cdot, t, \cdot), \partial_{y \xi}^{2} a\right\rangle_{\mathcal{S}^{\prime}, \mathcal{S}}-\frac{\epsilon}{2}\left\langle\partial_{y} \boldsymbol{\Theta} \mathcal{W}^{\epsilon}(\cdot, t, \cdot), \partial_{y \xi^{2}}^{3} a\right\rangle_{\mathcal{S}^{\prime}, \mathcal{S}}
$$

Passing all the derivatives of $\mathcal{W}^{\epsilon}$ with respect to $y$ or $\xi$ to the accompanying factors, we obtain

$$
\mathcal{I}^{\epsilon}=-\frac{\epsilon}{4}\left\langle, \partial_{y \xi^{2}}^{3} \boldsymbol{\Theta} a(y, \zeta)+\Theta \partial_{y \xi^{2}}^{3} a, \mathcal{W}^{\epsilon}(\cdot, t, \cdot)\right\rangle_{\mathcal{S}, \mathcal{S}^{\prime}},
$$

so that, taking into account that $\mathcal{W}^{\epsilon}$ is bounded in $\left(\mathcal{S}^{\prime}\left(\mathbb{R}_{y} \times \mathbb{R}_{\zeta}\right)\right)^{4}$ and that $\boldsymbol{\Theta}=\boldsymbol{\Theta}_{1}+\epsilon \boldsymbol{\Theta}_{0}$, with $\boldsymbol{\Theta}_{0}$ and $\boldsymbol{\Theta}_{1}$ regular enough so that $\boldsymbol{\Theta}_{i} a$ and $\partial_{y \xi^{2}}^{3} \boldsymbol{\Theta}_{i} a$ are in $\left(L^{\infty}\left(\mathbb{R}^{2}\right)\right)^{4}$, for all $i=0,1$ and $a \in \mathcal{S}\left(\mathbb{R}^{2}\right)$, we conclude that $\mathcal{I}^{\epsilon}=O(\epsilon)$.

By applying the Parseval identity in the left hand side of $(\mathrm{A} .15$, taking into account the above considerations on $\mathbf{A}^{\epsilon}$ and $\mathbf{B}^{\epsilon}$ (see $(\mathrm{A} .18)$ ), the fact that $\boldsymbol{\Theta}=\boldsymbol{\Theta}_{1}+\epsilon \boldsymbol{\Theta}_{0}$ and that $\boldsymbol{\Theta}_{1}^{*}=-\boldsymbol{\Theta}_{1}$, we conclude that the matrix $\mathcal{W}^{\epsilon}(y, t, \zeta)$ verifies the equation

$$
\begin{aligned}
\partial_{t} \mathcal{W}^{\epsilon} & =\frac{\boldsymbol{\Theta}_{1} \mathcal{W}^{\epsilon}-\mathcal{W}^{\epsilon} \boldsymbol{\Theta}_{1}}{\epsilon}+\left(\boldsymbol{\Theta}_{0} \mathcal{W}^{\epsilon}+\mathcal{W}^{\epsilon} \boldsymbol{\Theta}_{0}^{*}\right)+\frac{1}{2 i}\left(\left\{\boldsymbol{\Theta}_{1}, \mathcal{W}^{\epsilon}\right\}-\left\{\mathcal{W}^{\epsilon}, \boldsymbol{\Theta}_{1}\right\}\right) \\
& -\frac{1}{2 i}\left(\partial_{y \xi}^{2} \boldsymbol{\Theta}_{1} \mathcal{W}^{\epsilon}+\mathcal{W}^{\epsilon} \partial_{y \xi}^{2} \boldsymbol{\Theta}_{1}\right)+\epsilon \boldsymbol{\mathcal { R }}^{\epsilon},
\end{aligned}
$$

where $\mathcal{R}^{\epsilon}$ is bounded in $\left(\mathcal{S}^{\prime}\left(\mathbb{R}^{2}\right)\right)^{4}$. Observe that this is a similar equation to (6.12) in 15, excepting the term $-\left(\partial_{y \xi}^{2} \boldsymbol{\Theta}_{1} \mathcal{W}^{\epsilon}+\mathcal{W}^{\epsilon} \partial_{y \xi}^{2} \boldsymbol{\Theta}_{1}\right) / 2 i$. This comes from the fact that in that case equation A.10 involves the 
Weyl operator $\boldsymbol{\Theta}^{w}\left(y, \epsilon \partial_{y}\right)$ instead of $\boldsymbol{\Theta}\left(y, \epsilon \partial_{y}\right)$ (for the definition of the Weyl operator, see formula (1.4) in [15]). Of course, $\mathcal{W}^{\epsilon}\left[\left(\boldsymbol{\Theta}\left(y, \epsilon \partial_{y}\right)-\boldsymbol{\Theta}^{w}\left(y, \epsilon \partial_{y}\right)\right) \mathbf{u}^{\epsilon}, \mathbf{v}^{\epsilon}\right] \rightarrow 0$ as $\epsilon \rightarrow 0$ in $\mathcal{S}^{\prime}\left(\mathbb{R}^{2}\right)$, for all vector functions $\mathbf{u}^{\epsilon}, \mathbf{v}^{\epsilon}$ bounded in $\left(L^{2}(\mathbb{R})\right)^{2}$ as $\epsilon \rightarrow 0$. Due to the fact that the operator $\boldsymbol{\Theta}\left(y, \epsilon \partial_{y}\right)$ in A.10 is multiplied by a factor $\epsilon^{-1}$, the additional term in A.19) with respect to (6.12) in [15] comes from the fact that $\mathcal{W}^{\epsilon}\left[\epsilon^{-1}\left(\boldsymbol{\Theta}\left(y, \epsilon \partial_{y}\right)-\boldsymbol{\Theta}^{w}\left(y, \epsilon \partial_{y}\right)\right) \mathbf{u}^{\epsilon}, \mathbf{v}^{\epsilon}\right]$ is not at all trivial as $\epsilon \rightarrow 0$.

Step II. Equation A.29) of the projections of the Wigner measure matrix on the Fourier modes of $\boldsymbol{\Theta}_{1}(y, \xi)$. Let us denote by $\mathcal{W}(y, t, \xi)$ the weak limit of the Wigner transform matrix $\mathcal{W}^{\epsilon}(y, t, \xi)$. Remark the following relation between the limit $\mathcal{W}$ in Theorem 2.2 which is a scalar quantity and $\mathcal{W}$ :

$$
\mathcal{W}=\lim _{\epsilon \rightarrow 0}\left(\mathcal{W}^{\epsilon}\left[w^{\epsilon}\right]+\mathcal{W}^{\epsilon}\left[\tilde{w}^{\epsilon}\right]\right)=\lim _{\epsilon \rightarrow 0} \operatorname{tr}\left(\mathcal{W}^{\epsilon}\right)=\operatorname{tr}(\mathcal{W})
$$

Since the first term on the right hand side of A.19 is of order $\epsilon^{-1}$, we get directly from A.19

$$
\boldsymbol{\Theta}_{1}(y, \xi) \mathcal{W}(y, t, \xi)=\mathcal{W}(y, t, \xi) \boldsymbol{\Theta}_{1}(y, \xi), \quad \forall y, \xi \in \mathbb{R} .
$$

This is the analogous of $(A .9)$ in the first proof of Theorem 2.2 Remark that, for $\xi=0, \boldsymbol{\Theta}_{1}(y, \xi)$ is the null matrix, so that A.21 becomes an obvious identity from which one cannot get information about $\mathcal{W}$.

Set $\mathcal{W}^{\epsilon, \pm}(y, t, \xi):=\boldsymbol{\Delta}^{ \pm} \mathcal{W}^{\epsilon}(y, t, \xi) \boldsymbol{\Delta}^{ \pm}$, where $\mathcal{W}^{\epsilon}$ is the Wigner transform matrix in A.19) and $\mathcal{W}^{ \pm}:=$ $\lim _{\epsilon \rightarrow 0} \mathcal{W}^{\epsilon, \pm}$. Let us multiply A.19 to the left and to the right by $\boldsymbol{\Delta}^{ \pm}$. In this way, the left hand side of A.19 is transformed into $\partial_{t} \mathcal{W}^{\epsilon, \pm}(y, t, \xi)$. Remark that, by using the decomposition A.12 of the matrix $\boldsymbol{\Theta}_{1}(y, \xi)$, the first and the second identities in A.13, we obtain

$$
\boldsymbol{\Delta}^{ \pm} \boldsymbol{\Theta}_{1}(y, \xi) \mathcal{W}^{\epsilon}(y, t, \xi) \boldsymbol{\Delta}^{ \pm}=\boldsymbol{\Delta}^{ \pm} \mathcal{W}^{\epsilon}(y, t, \xi) \boldsymbol{\Theta}_{1}(y, \xi) \boldsymbol{\Delta}^{ \pm}=i \lambda_{c, \omega}^{ \pm}(y, \xi) \mathcal{W}^{\epsilon, \pm}(y, t, \xi),
$$

with $\omega(\xi)=\xi$. Thus, under this process of multiplication of A.19 by $\boldsymbol{\Delta}^{ \pm}$, the term of order $\epsilon^{-1}$ on the right hand side of A.19) vanishes. Now, let us remark that, using the last and the first identities in (A.13), we obtain

$$
\boldsymbol{\Delta}^{ \pm} \boldsymbol{\Theta}_{0}(y, \xi) \mathcal{W}^{\epsilon}(y, t, \xi) \boldsymbol{\Delta}^{ \pm}=\boldsymbol{\Delta}^{ \pm} \boldsymbol{\Theta}_{0}(y, \xi) \boldsymbol{\Delta}^{ \pm} \mathcal{W}^{\epsilon, \pm}(y, t, \xi)+\boldsymbol{\Delta}^{ \pm} \boldsymbol{\Theta}_{0}(y, \xi) \boldsymbol{\Delta}^{\mp} \mathcal{W}^{\epsilon}(y, t, \xi) \boldsymbol{\Delta}^{ \pm} .
$$

We also get the similar identity $\boldsymbol{\Delta}^{ \pm} \mathcal{W}^{\epsilon} \Theta_{0}^{*} \boldsymbol{\Delta}^{ \pm}=\mathcal{W}^{\epsilon, \pm} \boldsymbol{\Delta}^{ \pm} \boldsymbol{\Theta}_{0}^{*} \boldsymbol{\Delta}^{ \pm}+\boldsymbol{\Delta}^{ \pm} \mathcal{W}^{\epsilon} \boldsymbol{\Delta}^{\mp} \boldsymbol{\Theta}_{0}^{*} \boldsymbol{\Delta}^{ \pm}$. An easy computation yields

$$
\boldsymbol{\Delta}^{ \pm} \boldsymbol{\Theta}_{0}(y, \xi) \boldsymbol{\Delta}^{ \pm}=\boldsymbol{\Delta}^{ \pm} \boldsymbol{\Theta}_{0}^{*}(y, \xi) \boldsymbol{\Delta}^{ \pm}= \pm \frac{1}{2}(d(y)+e(y)) \boldsymbol{\Delta}^{ \pm}
$$

where the functions $d$ and $e$ have been introduced in 2.16. Thus, the second term in the right hand side of A.19 becomes:

$$
\boldsymbol{\Delta}^{ \pm}\left(\boldsymbol{\Theta}_{0} \mathcal{W}^{\epsilon}+\mathcal{W}^{\epsilon} \boldsymbol{\Theta}_{0}^{*}\right) \boldsymbol{\Delta}^{ \pm}= \pm(d+e) \mathcal{W}^{\epsilon, \pm}+\boldsymbol{\Delta}^{ \pm} \boldsymbol{\Theta}_{0} \boldsymbol{\Delta}^{\mp} \mathcal{W}^{\epsilon} \boldsymbol{\Delta}^{ \pm}+\boldsymbol{\Delta}^{ \pm} \mathcal{W}^{\epsilon} \boldsymbol{\Delta}^{\mp} \boldsymbol{\Theta}_{0}^{*} \boldsymbol{\Delta}^{ \pm}
$$

In what follows, we show that the last two terms in the right hand side of $(\mathrm{A} .25)$ converge to $\mathbf{0}_{2}$ as $\epsilon \rightarrow 0$ for $\xi \neq 0$. To this aim, we multiply A.21 by $\boldsymbol{\Delta}^{\mp}$ to the left and by $\boldsymbol{\Delta}^{ \pm}$to the right. Taking into account A.12 and the first two identities in A.13, we obtain $\left(\lambda_{c, \xi}^{ \pm}(y, \xi)-\lambda_{c, \xi}^{\mp}(y, \xi)\right) \boldsymbol{\Delta}^{\mp} \boldsymbol{W}(y, t, \xi) \boldsymbol{\Delta}^{ \pm}=\mathbf{0}_{2}$, and, by taking into account the fact that, for $\xi \neq 0, \lambda_{c, \xi}^{ \pm}(y, \xi) \neq \lambda_{c, \xi}^{\mp}(y, \xi)$, we finally get

$$
\boldsymbol{\Delta}^{\mp} \mathcal{W} \boldsymbol{\Delta}^{ \pm}=\mathbf{0}_{2} \text {. }
$$

Due to A.12, to the fact that the projectors $\boldsymbol{\Delta}^{ \pm}$do not depend on anyone of the two variables $y$ and $\xi$ and to A.13), we obtain the following two identities concerning the third and the fourth terms in A.19):

$$
\frac{1}{2 i} \boldsymbol{\Delta}^{ \pm}\left(\left\{\boldsymbol{\Theta}_{1}, \mathcal{W}^{\epsilon}\right\}-\left\{\mathcal{W}^{\epsilon}, \boldsymbol{\Theta}_{1}\right\}\right) \boldsymbol{\Delta}^{ \pm}=\left\{\lambda_{c, \xi}^{ \pm}, \mathcal{W}^{\epsilon, \pm}\right\}
$$

and

$$
\frac{1}{2 i} \boldsymbol{\Delta}^{ \pm}\left(\partial_{y \xi}^{2} \boldsymbol{\Theta}_{1} \mathcal{W}^{\epsilon}+\mathcal{W}^{\epsilon} \partial_{y \xi}^{2} \boldsymbol{\Theta}_{1}\right) \boldsymbol{\Delta}^{ \pm}=\partial_{y \xi}^{2} \lambda_{c, \xi}^{ \pm} \mathcal{W}^{\epsilon, \pm} .
$$

Recollecting A.22 and A.25 A.28, we obtain that the matrix $\mathcal{W}^{ \pm}=\lim _{\epsilon \rightarrow 0} \mathcal{W}^{\epsilon, \pm}$ verifies the problem $\partial_{t} \mathcal{W}^{ \pm}= \pm(d(y)+e(y)) \mathcal{W}^{ \pm}+\left\{\lambda_{c, \omega}^{ \pm}, \mathcal{W}^{ \pm}\right\}-\partial_{y \xi}^{2} \lambda_{c, \xi}^{ \pm} \mathcal{W}^{ \pm}$, with $\omega(\xi)=\xi$. Using 2.16 and the fact that 
$\lambda_{c, \omega}^{+}=-\lambda_{c, \omega}^{-}=c(y) \xi$, we see that the first and the third terms on the right hand side of this equation cancel, so that the equation for $\mathcal{W}^{ \pm}$simplifies to

$$
\partial_{t} \mathcal{W}^{ \pm}=\left\{\lambda_{c, \omega}^{ \pm}, \mathcal{W}^{ \pm}\right\}
$$

Remark that by applying the trace operator to $A .29$, we obtain that $\mathcal{W}^{ \pm}=\operatorname{tr}\left(\boldsymbol{W}^{ \pm}\right)$verifies equation 2.20). By passing to the limit as $\epsilon \rightarrow 0$ in the identity $\mathcal{W}^{\epsilon, \pm}=\boldsymbol{\Delta}^{ \pm} \mathcal{W}^{\epsilon} \boldsymbol{\Delta}^{ \pm}$, we obtain that $\mathcal{W}^{ \pm}=\boldsymbol{\Delta}^{ \pm} \mathcal{W} \boldsymbol{\Delta}^{ \pm}$. We remark the following identities:

$$
\mathcal{W}=\operatorname{tr}(\mathcal{W})=\operatorname{tr}\left(\mathcal{W}^{+}\right)+\operatorname{tr}\left(\mathcal{W}^{-}\right)=\mathcal{W}^{+}+\mathcal{W}^{-},
$$

where $\mathcal{W}=\lim _{\epsilon \rightarrow 0}\left(\mathcal{W}^{\epsilon}\left[w^{\epsilon}\right]+\mathcal{W}^{\epsilon}\left[\tilde{w}^{\epsilon}\right]\right)$ is the Wigner measure introduced in the statement of Theorem 2.2 and $\mathcal{W}^{ \pm}=\lim _{\epsilon \rightarrow 0} \mathcal{W}^{\epsilon}\left[\left(w^{\epsilon} \pm \tilde{w}^{\epsilon}\right) / \sqrt{2}\right]$. This concludes the proof of Theorem 2.2

\section{A.3. Proof of Proposition 3.1.}

Remark 6. Using the change of variable $\widetilde{u}(\widetilde{y}, t)=u(y, t)$, with $\widetilde{y}=H(y)$ and $H^{\prime}(y)=\varrho(y)$, the transport equation with variable coefficients (1.1) becomes the transport equation with constant coefficients below:

$$
\partial_{t} \widetilde{u}(\widetilde{y}, t)+\partial_{\widetilde{y}} \widetilde{u}(\widetilde{y}, t)=0, \widetilde{y} \in \mathbb{R}, t>0, \quad \widetilde{u}(\widetilde{y}, 0)=\widetilde{u}^{0}(\widetilde{y}):=u^{0}\left(H^{-1}(\widetilde{y})\right),
$$

for which the solution is $\widetilde{u}(\widetilde{y}, t)=\widetilde{u}^{0}(\widetilde{y}-t)=u^{0}\left(H^{-1}(\widetilde{y}-t)\right)$. Then the solution of (1.1) is given by $u(y, t)=u^{0}\left(H^{-1}(H(y)-t)\right)$.

We use Lax-Richtmyer Theorem (cf. [19]), guaranteing that a numerical scheme is convergent if and only if both its consistency and stability properties hold. The stability of (1.6) means the existence of an uniform constant $C>0$ as $h \rightarrow 0\left(C:=g_{d}^{-} \varrho^{-}\right.$in our case) such that (here, $\left.\mathbf{f}^{h, 1} \odot \mathbf{f}^{h, 2}:=\left(f_{j}^{1} f_{j}^{2}\right)_{j \in \mathbb{Z}}\right)$

$$
\left(\partial^{h} g\left(\mathbf{x}^{h}\right) \odot \varrho\left(g\left(\mathbf{x}^{h}\right)\right) \odot \partial_{t} \mathbf{f}^{h}(t)-\partial^{h} \mathbf{f}^{h}(t), \mathbf{f}^{h}(t)\right)_{\ell_{h}^{2}} \geq C \partial_{t}\left(\left\|\mathbf{f}^{h}(t)\right\|_{\ell_{h}^{2}}^{2}\right), \forall \mathbf{f}^{h}(t) \in \ell_{h}^{2}, \forall t>0 .
$$

The consistency means to consider a solution $u(\cdot, t)$ of 1.1$)$ belonging to $C_{c}^{2}\left(\mathbb{R}_{x}\right)$ for all $t>0$ and to plug it in the numerical scheme (1.6) to obtain (by Taylor expansions):

$$
\varrho\left(g_{j}\right) \partial_{t} u\left(g_{j}, t\right)+\frac{u\left(g_{j+1}, t\right)-u\left(g_{j-1}, t\right)}{g_{j+1}-g_{j-1}}=r_{j}(t)
$$

where

$$
r_{j}(t):=\frac{1}{2} \frac{\left(g_{j+1}-g_{j}\right)^{2}}{\left(g_{j+1}-g_{j-1}\right)} \partial_{y}^{2} u\left(\theta_{j+1 / 2}, t\right)-\frac{1}{2} \frac{\left(g_{j}-g_{j-1}\right)^{2}}{\left(g_{j+1}-g_{j-1}\right)} \partial_{y}^{2} u\left(\theta_{j-1 / 2}, t\right)
$$

and $\theta_{j+1 / 2} \in\left(g_{j}, g_{j+1}\right)$, for all $j \in \mathbb{Z}$ and all $t>0$.

Using the hypothesis on $g$, we obtain the following estimate on the residual $\mathbf{r}^{h}(t):=\left(r_{j}(t)\right)_{j \in \mathbb{Z}}$ :

$$
\left|\partial^{h} g_{j} r_{j}(t)\right| \leq \frac{h}{4}\left(g_{d}^{+}\right)^{2}\left(\left|\partial_{y}^{2} u\left(\theta_{j+1 / 2}, t\right)\right|+\left|\partial_{y}^{2} u\left(\theta_{j-1 / 2}, t\right)\right|\right), \forall j \in \mathbb{Z}, \forall t>0 .
$$

Let us remark that the error $\mathbf{e}^{h}(t):=u\left(g\left(\mathbf{x}^{h}\right), t\right)-\mathbf{u}^{h}(t)$ satisfies the problem

$$
\varrho\left(g\left(x_{j}\right)\right) \partial_{t} e_{j}(t)+\frac{e_{j+1}(t)-e_{j-1}(t)}{g_{j+1}-g_{j-1}}=r_{j}(t), \quad e_{j}(0)=0, \forall j \in \mathbb{Z}, t>0 .
$$

By multiplying A.35 by $h \partial^{h} g_{j} e_{j}(t)$, adding in $j \in \mathbb{Z}$ and using the stability estimate A.32, we obtain

$$
g_{d}^{-} \varrho^{-} \partial_{t}\left(\left\|\mathbf{e}^{h}(t)\right\|_{\ell_{h}^{2}}^{2}\right) \leq\left\|\mathbf{e}^{h}(t)\right\|_{\ell_{h}^{2}}\left\|\partial^{h} \mathbf{g}^{h} \odot \mathbf{r}^{h}(t)\right\|_{\ell^{2}},
$$

form where, using the hypothesis on $g$, we have

$$
\partial_{t}\left(\left\|\mathbf{e}^{h}(t)\right\|_{\ell^{2}}\right) \leq \frac{1}{2 g_{d}^{-} \varrho^{-}}\left\|\partial^{h} \mathbf{g}^{h} \odot \mathbf{r}^{h}(t)\right\|_{\ell^{2}}
$$

or, taking into account the fact that $\mathbf{e}^{h}(0)=0$,

$$
\left\|\mathbf{e}^{h}(t)\right\|_{\ell^{2}} \leq \frac{1}{2 g_{d}^{-} \varrho^{-}} \int_{0}^{t}\left\|\partial^{h} \mathbf{g}^{h} \odot \mathbf{r}^{h}(s)\right\|_{\ell^{2}} d s \leq \frac{t}{2 g_{d}^{-} \varrho^{-}} \sup _{s \in[0, t]}\left\|\partial^{h} \mathbf{g}^{h} \odot \mathbf{r}^{h}(s)\right\|_{\ell^{2}} .
$$


From Remark 6, we see that

$$
\begin{aligned}
u_{y y}\left(\theta_{j \pm 1 / 2}, t\right) & =\left(u^{0}\right)^{\prime \prime}\left(H^{-1}\left(H\left(\theta_{j \pm 1 / 2}\right)+t\right)\right)\left(\frac{\varrho\left(\theta_{j \pm 1 / 2}\right)}{\varrho\left(H\left(\theta_{j \pm 1 / 2}\right)+t\right)}\right)^{2} \\
& +\left(u^{0}\right)^{\prime}\left(H^{-1}\left(H\left(\theta_{j \pm 1 / 2}\right)+t\right)\right) \frac{\varrho^{\prime}\left(\theta_{j \pm 1 / 2}\right) \varrho\left(H\left(\theta_{j \pm 1 / 2}\right)+t\right)-\left(\varrho\left(\theta_{j \pm 1 / 2}\right)\right)^{2} \varrho^{\prime}\left(H\left(\theta_{j \pm 1 / 2}\right)+t\right)}{\left(\varrho\left(H\left(\theta_{j \pm 1 / 2}\right)+t\right)\right)^{2}},
\end{aligned}
$$

so that, using the hypothesis on the coefficient $\varrho$, we obtain

$$
\left|u_{y y}\left(\theta_{j \pm 1 / 2}, t\right)\right| \leq\left|\left(u^{0}\right)^{\prime \prime}\left(H^{-1}\left(H\left(\theta_{j \pm 1 / 2}\right)+t\right)\right)\right|\left(\frac{\varrho^{+}}{\varrho^{-}}\right)^{2}+\left|\left(u^{0}\right)^{\prime}\left(H^{-1}\left(H\left(\theta_{j \pm 1 / 2}\right)+t\right)\right)\right| \frac{\varrho^{+} \varrho_{d}^{+}\left(1+\varrho^{+}\right)}{\left(\varrho^{-}\right)^{2}}
$$

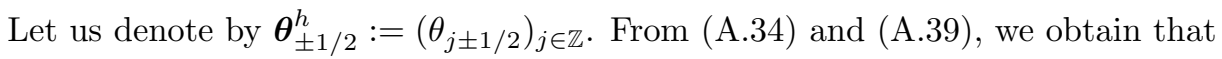

$$
\begin{aligned}
\left\|\partial^{h} \mathbf{g}^{h} \odot \mathbf{r}^{h}(s)\right\|_{\ell^{2}} & \leq \frac{h}{4}\left(g_{d}^{+}\right)^{2}\left(\left\|\partial_{y}^{2} u\left(\boldsymbol{\theta}_{1 / 2}^{h}, s\right)\right\|_{\ell^{2}}+\left\|\partial_{y}^{2} u\left(\boldsymbol{\theta}_{-1 / 2}^{h}, s\right)\right\|_{\ell^{2}}\right) \\
& \leq \frac{h}{4}\left(g_{d}^{+}\right)^{2}\left[\left(\frac{\varrho^{+}}{\varrho^{-}}\right)^{2}\left(\left\|\left(u^{0}\right)^{\prime \prime}\left(H^{-1}\left(H\left(\boldsymbol{\theta}_{1 / 2}^{h}\right)+s\right)\right)\right\|_{\ell^{2}}+\left\|\left(u^{0}\right)^{\prime \prime}\left(H^{-1}\left(H\left(\boldsymbol{\theta}_{-1 / 2}^{h}\right)+s\right)\right)\right\|_{\ell^{2}}\right)\right. \\
& \left.+\frac{\varrho^{+} \varrho_{d}^{+}\left(1+\varrho^{+}\right)}{\left(\varrho^{-}\right)^{2}}\left(\left\|\left(u^{0}\right)^{\prime}\left(H^{-1}\left(H\left(\boldsymbol{\theta}_{1 / 2}^{h}\right)+s\right)\right)\right\|_{\ell^{2}}+\left\|\left(u^{0}\right)^{\prime}\left(H^{-1}\left(H\left(\boldsymbol{\theta}_{-1 / 2}^{h}\right)+s\right)\right)\right\| \ell_{\ell^{2}}\right)\right] .
\end{aligned}
$$

For a function $f \in C_{c}(\mathbb{R})$, we have

$$
\begin{aligned}
\left.\left\|f\left(H^{-1}\left(H\left(\boldsymbol{\theta}_{ \pm 1 / 2}^{h}\right)+s\right)\right)\right\|_{\ell^{2}}\right) & \leq\|f\|_{L^{\infty}(\mathbb{R})}\left(h \sharp\left\{j \text { s.t. } H^{-1}\left(H\left(\theta_{j \pm 1 / 2}\right)+s\right) \in \operatorname{supp} f\right\}\right)^{1 / 2} \\
& \leq\|f\|_{L^{\infty}(\mathbb{R})}\left(\mathcal{L}\left(g^{-1}\left(H^{-1}(H(\operatorname{supp} f-s))\right)\right)\right)^{1 / 2},
\end{aligned}
$$

where $\mathcal{L}(A)$ is the Lebesgue measure of the set $A$.

The conclusion (3.4) follows by taking

$$
\begin{aligned}
C\left(t, g, \varrho, u^{0}\right) & :=\frac{t\left(g_{d}^{+}\right)^{2}}{4 g_{d}^{-} \varrho^{-}}\left[\left(\frac{\varrho^{+}}{\varrho^{-}}\right)^{2}\left\|\left(u^{0}\right)^{\prime \prime}\right\|_{L^{\infty}(\mathbb{R})}\left(\sup _{s \in[0, t]} \mathcal{L}\left(g^{-1}\left(H^{-1}\left(H\left(\operatorname{supp}\left(u^{0}\right)^{\prime \prime}-s\right)\right)\right)\right)\right)^{1 / 2}\right. \\
& \left.+\frac{\varrho^{+} \varrho_{d}^{+}\left(1+\varrho^{+}\right)}{\left(\varrho^{-}\right)^{2}}\left\|\left(u^{0}\right)^{\prime}\right\|_{L^{\infty}(\mathbb{R})}\left(\sup _{s \in[0, t]} \mathcal{L}\left(g^{-1}\left(H^{-1}\left(H\left(\operatorname{supp}\left(u^{0}\right)^{\prime}-s\right)\right)\right)\right)\right)^{1 / 2}\right]
\end{aligned}
$$

A.4. Proof of Proposition 3.4. As in the proof of Proposition 3.1 the error $\epsilon^{h}(t):=u\left(g_{j}, t\right)-u_{j}(t)$ solves the equation:

$$
\rho\left(g_{j}\right) \partial_{t}^{2} \epsilon_{j}-\frac{\sigma\left(g\left(x_{j+1 / 2}\right)\right) \frac{\epsilon_{j+1}(t)-\epsilon_{j}(t)}{g_{j+1}-g_{j}}-\sigma\left(g\left(x_{j-1 / 2}\right)\right) \frac{\epsilon_{j}(t)-\epsilon_{j-1}(t)}{g_{j}-g_{j-1}}}{\frac{g_{j+1}-g_{j-1}}{2}}=r_{j}(t), \quad \epsilon_{j}(0)=\epsilon_{j, t}(0)=0
$$

for all $j \in \mathbb{Z}$ and $t>0$. Here the residual $r_{j}(t)$ below is the error obtained in the consistency analysis when the solution $u\left(g_{j}, t\right)$ of the continuous equation $[1.4)$ is introduced in the discrete equation $(1.6)$ :

$$
r_{j}(t):=r_{j}^{1}(t)+\cdots+r_{j}^{7}(t),
$$

where

$$
\begin{gathered}
r_{j}^{1}(t):=\frac{\sigma\left(g_{j}\right) \sum_{ \pm}( \pm)\left(g_{j \pm 1}-g_{j}\right)^{2} \partial_{y}^{3} u\left(\theta_{j \pm 1 / 2}, t\right)}{3\left(g_{j+1}-g_{j-1}\right)}, r_{j}^{2}(t):=-\sigma^{\prime}\left(g_{j}\right) \partial_{y} u\left(g_{j}, t\right) \frac{g_{j+1}-2 g_{j+1 / 2}+2 g_{j-1 / 2}-g_{j-1}}{g_{j+1}-g_{j-1}}, \\
r_{j}^{3}(t):=\sigma^{\prime}\left(g_{j}\right) \partial_{y}^{2} u\left(g_{j}, t\right) \frac{\sum_{ \pm}( \pm)\left(g_{j \pm 1 / 2}-g_{j}\right)\left(g_{j \pm 1}-g_{j}\right)}{g_{j+1}-g_{j-1}} \\
r_{j}^{4}(t):=\frac{\sum_{ \pm}( \pm)\left(g_{j \pm 1 / 2}-g_{j}\right)\left(g_{j \pm 1}-g_{j}\right)^{2} \sigma^{\prime}\left(g_{j}\right) \partial_{y}^{3} u\left(\theta_{j \pm 1 / 2}, t\right)}{3\left(g_{j+1}-g_{j-1}\right)}, r_{j}^{5}(t):=\frac{\sum_{ \pm}( \pm)\left(g_{j \pm 1 / 2}-g_{j}\right)^{2} \sigma^{\prime \prime}\left(\theta_{j \pm 1 / 4}\right) \partial_{y} u\left(g_{j}, t\right)}{g_{j+1}-g_{j-1}},
\end{gathered}
$$




$$
\begin{aligned}
r_{j}^{6}(t) & :=\frac{\sum_{ \pm}( \pm)\left(g_{j \pm 1 / 2}-g_{j}\right)^{2}\left(g_{j \pm 1}-g_{j}\right) \sigma^{\prime \prime}\left(\theta_{j \pm 1 / 4}\right) \partial_{y}^{2} u\left(g_{j}, t\right)}{2\left(g_{j+1}-g_{j-1}\right)}, \\
r_{j}^{7}(t) & :=\frac{\sum_{ \pm}( \pm)\left(g_{j \pm 1 / 2}-g_{j}\right)^{2}\left(g_{j \pm 1}-g_{j}\right)^{2} \sigma^{\prime \prime}\left(\theta_{j \pm 1 / 4}\right) \partial_{y}^{3} u\left(\theta_{j \pm 1 / 2}, t\right)}{6\left(g_{j+1}-g_{j-1}\right)},
\end{aligned}
$$

with $g_{j-1 / 2}<\theta_{j-1 / 4}<g_{j}<\theta_{j+1 / 4}<g_{j+1 / 2}$ and $g_{j-1}<\theta_{j-1 / 2}<g_{j}<\theta_{j+1 / 2}<g_{j+1}$.

Let us remark that, using the hypothesis on $g$ and $\sigma$, we obtain

$$
\begin{gathered}
\left|\partial^{h} g_{j} r_{j}^{1}(t)\right| \leq \frac{\sigma^{+}\left(g_{d}^{+}\right)^{2}}{6} h \sum_{ \pm}\left|\partial_{y}^{3} u\left(\theta_{j \pm 1 / 2}, t\right)\right|,\left|\partial^{h} g_{j} r_{j}^{2}(t)\right| \leq \frac{\sigma_{d}^{+} g_{d d}^{+}}{4} h\left|\partial_{y} u\left(g_{j}, t\right)\right|, \\
\left|\partial^{h} g_{j} r_{j}^{3}(t)\right| \leq \frac{\sigma_{d}^{+}\left(g_{d}^{+}\right)^{2}}{2} h\left|\partial_{y}^{2} u\left(g_{j}, t\right)\right|, \quad\left|\partial^{h} g_{j} r_{j}^{4}(t)\right| \leq \frac{\left(g_{d}^{+}\right)^{3} \sigma_{d}^{+}}{12} h^{2} \sum_{ \pm}\left|\partial_{y}^{3} u\left(\theta_{j \pm 1 / 2}, t\right)\right|, \\
\left|\partial^{h} g_{j} r_{j}^{5}(t)\right| \leq \frac{\sigma_{d d}^{+}\left(g_{d}^{+}\right)^{2}}{4} h\left|\partial_{y} u\left(g_{j}, t\right)\right|, \quad\left|\partial^{h} g_{j} r_{j}^{6}(t)\right| \leq \frac{\sigma_{d d}^{+}\left(g_{d}^{+}\right)^{3}}{8} h^{2}\left|\partial_{y}^{2} u\left(g_{j}, t\right)\right|,
\end{gathered}
$$

and

$$
\left|\partial^{h} g_{j} r_{j}^{7}(t)\right| \leq \frac{\sigma_{d d}^{+}\left(g_{d}^{+}\right)^{4}}{48} h^{3} \sum_{ \pm}\left|\partial_{y}^{3} u\left(\theta_{j \pm 1 / 2}, t\right)\right| .
$$

By multiplying A.41 by $h \partial^{h} g_{j} \epsilon_{j}^{\prime}(t)$, we obtain

$$
\begin{aligned}
& 2 \min \left\{\sqrt{g_{d}^{-} \rho^{-}}, \sqrt{\frac{\sigma^{-}}{g_{d}^{+}}}\right\}\left\|\left(\boldsymbol{\epsilon}^{h}(t), \partial_{t} \boldsymbol{\epsilon}^{h}(t)\right)\right\|_{\dot{\hbar} \times \ell_{h}^{2}} \leq 2\left(2 \mathcal{E}_{\rho, \sigma, g}^{h}\left(\boldsymbol{\epsilon}^{h}(t), \partial_{t} \boldsymbol{\epsilon}^{h}(t)\right)\right)^{1 / 2} \\
& \leq \int_{0}^{t}\left(h \sum_{j \in \mathbb{Z}} \frac{\partial^{h} g_{j}}{\rho\left(g_{j}\right)}\left|r_{j}(s)\right|^{2}\right)^{1 / 2} d s \\
& \leq \frac{t}{\sqrt{\rho^{-} g_{d}^{-}}} \sup _{s \in[0, t]}\left(\left\|\partial^{h} \mathbf{g}^{h} \odot \mathbf{r}^{h}(s)\right\|_{\ell^{\infty}}\left(h \sharp\left\{j \text { s.t. } r_{j}(s) \neq 0\right\}\right)^{1 / 2}\right) .
\end{aligned}
$$

Remark that

$$
\left\|\partial^{h} \mathbf{g}^{h} \odot \mathbf{r}^{h}(s)\right\|_{\ell^{\infty}} \leq\left(C_{1} h+C_{2} h^{2}+C_{3} h^{3}\right)\|u(\cdot, s)\|_{W^{3, \infty}(\mathbb{R})} .
$$

Let us introduce the function

$$
F(y)=\int_{0}^{y} \sqrt{\frac{\rho(z)}{\sigma(z)}} d z .
$$

For all $x \in \mathbb{R}$ and $s \in \mathbb{R}_{+}$, set $x^{s, \pm}:=F^{-1}(F(x) \pm s)$. Let us remark that for the continuous wave equation (1.4), the space component $y_{ \pm}(t)$ of the two families of characteristics in 2.4 can be found independently on the phase variable $\xi_{ \pm}(t)$ and is precisely $y_{ \pm}(t)=y^{t, \pm}=F^{-1}(F(y) \pm t)$, with $F$ as in (A.44).

The following result shows that if the initial data $\left(u^{0}, u^{1}\right)$ in $(1.4)$ is compactly supported in $(a, b)$, then $u(\cdot, s)$ is supported in $\left.\left(a^{s,-}, b^{s,+}\right)\right)$,

Lemma A.1. Let $[a, b]=\left(\right.$ suppu $u^{0} \cup$ suppu $\left.{ }^{1}\right)$ and $\left(y_{0}, y_{1}\right) \subset \mathbb{R} \backslash[a, b]$ be an interval in the complementary of the support of the initial data $\left(u^{0}, u^{1}\right)$ in (1.4). Then, for all $s<T_{F}:=\left(F\left(y_{1}\right)-F\left(y_{0}\right)\right) / 2$,

$$
\int_{y_{0}^{s,+}}^{y_{1}^{s,-}}\left(\rho(y)\left|\partial_{t} u(y, s)\right|^{2}+\sigma(y)\left|\partial_{y} u(y, s)\right|^{2}\right) d y=0 .
$$


By choosing $\left(y_{0}^{\alpha}, y_{1}^{\alpha}\right) \subset \mathbb{R} \backslash[a, b]$ such that $\left(y_{0}^{\alpha, s,+}, y_{1}^{\alpha, s,-}\right)_{\alpha}$ is a partition of $\mathbb{R} \backslash\left[a^{s,-}, b^{s,+}\right]$, we get

$$
\int_{\mathbb{R} \backslash\left[a^{s,-}, b^{s,+}\right]}\left(\rho(y)\left|\partial_{t} u(y, s)\right|^{2}+\sigma(y)\left|\partial_{y} u(y, s)\right|^{2}\right) d y=0,
$$

so that, using the decay properties of the solution $u(\cdot, s)$ of $(1.4)$ at infinity, we obtain that $u(\cdot, s) \equiv 0$ in $\mathbb{R} \backslash\left[a^{s,-}, b^{s,+}\right]$ for all $s \geq 0$, meaning that $\operatorname{supp} u(\cdot, s) \subseteq\left[a^{s,-}, b^{s,+}\right]$. Thus

$$
h \sharp\left\{j \text { s.t. } r_{j}(s) \neq 0\right\} \leq h \sharp\left\{j \text { s.t. } u\left(g_{j}, s\right) \neq 0\right\}=h \sharp\left\{j \text { s.t. } g_{j} \in\left[a^{s,-}, b^{s,+}\right]\right\}=\mathcal{L}\left(g^{-1}\left(\left[a^{s,-}, b^{s,+}\right]\right)\right) \text {. }
$$

It is well-known that if the initial data $\left(u^{0}, u^{1}\right)$ in 1.4 belong to $\dot{H}^{k} \times \dot{H}^{k-1}(\mathbb{R})$ and is compactly supported, then the solution $u(\cdot, t)$ belongs to $\dot{H}^{k}(\mathbb{R})$ and, as we saw, it is compactly supported. Then $u(\cdot, s) \in H^{k}(\mathbb{R})$. In order to guarantee that $u(\cdot, s) \in W^{3, \infty}(\mathbb{R})$, we have to impose $H^{k}(\mathbb{R}) \subseteq W^{3, \infty}(\mathbb{R})$, which, by the Sobolev embedding, holds if $k \geq 3+1 / 2$.

We conclude the proof of (3.17) by plugging A.43 and A.46 into A.42.

Proof of Lemma A.1. Let us consider the curved trapezoid $\mathcal{T}=\mathcal{T}\left(y_{0}, y_{1}, F, s\right):=\left\{y \in\left[y_{0}^{t,+}, y_{1}^{t,-}\right], t \in[0, s]\right\}$ (see Fig. 11, multiply equation (1.4) by $\partial_{t} u(y, t)$ and integrate in $\mathcal{T}$.

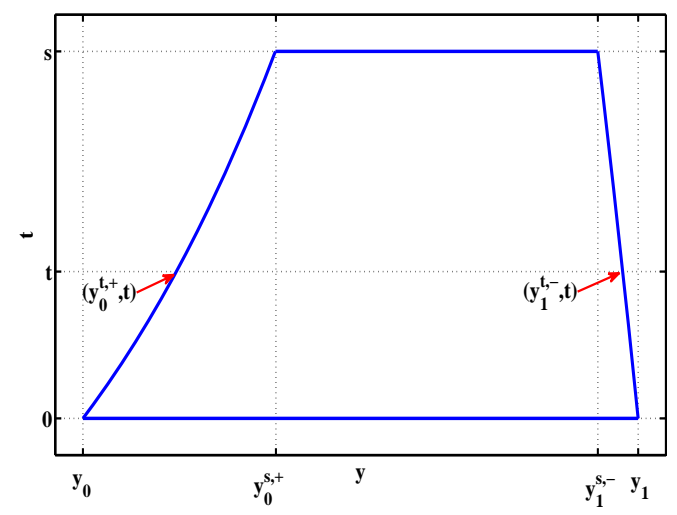

FiguRE 11. The curved trapezoid $\mathcal{T}$ is the region inside the blue curve. 
By integrations by parts, we obtain only integrals on the boundary of the trapezoid. More precisely, by integrating $\rho(y) \partial_{t}^{2} u(y, t) \partial_{t} u(y, t)$, we obtain

$$
\begin{aligned}
\int_{\mathcal{T}} \rho(y) u_{t t}(y, t) u_{t}(y, t) d y d t & =\frac{1}{2} \int_{0}^{s} \int_{y_{0}^{t,+}}^{y_{1}^{t,-}} \rho(y) \partial_{t}\left(\left|u_{t}(y, t)\right|^{2}\right) d y d t \\
& =\frac{1}{2}\left[\int_{y_{0}}^{y_{0}^{s,+}} F(y)-F\left(y_{0}\right)\right. \\
& \left.=\frac{1}{2} \int_{y_{0}}^{y_{1}^{s,-}} \rho(y) \int_{y_{0}^{s,+}}^{s} \int_{0}^{s}+\int_{y_{1}^{s,-}}^{y_{1}} \int_{0}^{F\left(y_{1}\right)-F(y)}\right] \rho(y) \partial_{t}\left(\left|u_{t}(y, t)\right|^{2}\right) d t d y \\
& +\frac{1}{2} \int_{y_{1}^{s,-}}^{y_{1}} \rho(y)\left|\partial_{t} u\left(y, F(y)-F\left(y_{0}\right)\right)\right|^{2} d y+\frac{1}{2} \int_{y_{0}^{s,+}}^{y_{1}^{s,-}} \rho(y)\left|\partial_{y} u(y, s)\right|^{2} d y
\end{aligned}
$$

By integrating $\partial_{y}\left(\sigma(y) \partial_{y} u\right)(y, t) \partial_{t} u(y, t)$, we obtain

$$
\begin{aligned}
\int_{\mathcal{T}} \partial_{y}\left(\sigma(y) \partial_{y} u\right)(y, t) \partial_{t} u(y, t) d y d t & =\int_{0}^{s} \int_{y_{0}^{t,+}}^{s} \partial_{y}\left(\sigma(y) \partial_{y} u\right)(y, t) \partial_{t} u(y, t) d y d t \\
& =\mathcal{I}_{3}-\mathcal{I}_{5}-\int_{\mathcal{T}} \sigma(y) \partial_{y} u(y, t) \partial_{y t}^{2} u(y, t) d y d t
\end{aligned}
$$

where

$$
\mathcal{I}_{3}:=\int_{0}^{s} \sigma\left(y_{1}^{t,-}\right) \partial_{y} u\left(y_{1}^{t,-}, t\right) \partial_{t} u\left(y_{1}^{t,-}, t\right) d t \text { and } \mathcal{I}_{5}:=\int_{0}^{s} \sigma\left(y_{0}^{t,+}\right) \partial_{y} u\left(y_{0}^{t,+}, t\right) \partial_{t} u\left(y_{0}^{t,+}, t\right) d t .
$$

Since $\sigma(y) \partial_{y} u(y, t) \partial_{y t}^{2} u(y, t)=\sigma(y) \partial_{t}\left(\left|\partial_{y} u(y, t)\right|^{2}\right) / 2$, the last term in the right hand side of A.48 can be treated similarly to A.47). Finally, we obtain

$$
0 \leq \frac{1}{2} \int_{y_{0}^{s,+}}^{y_{1}^{s,-}}\left(\rho(y)\left|\partial_{t} u(y, t)\right|^{2}+\sigma(y)\left|\partial_{y} u(y, t)\right|^{2}\right) d y=\mathcal{I}_{1}-\mathcal{I}_{2}-\mathcal{I}_{3}-\mathcal{I}_{4}+\mathcal{I}_{5}
$$

where

$$
\begin{gathered}
\mathcal{I}_{1}:=\frac{1}{2} \int_{y_{0}}^{y_{1}}\left(\rho(y)\left|u^{1}(y)\right|^{2}+\sigma(y)\left|\left(u^{0}\right)^{\prime}(y)\right|^{2}\right) d y \\
\mathcal{I}_{2}:=\frac{1}{2} \int_{y_{0}}^{y_{0}^{s,+}}\left(\rho(y)\left|\partial_{t} u\left(y, F(y)-F\left(y_{0}\right)\right)\right|^{2}+\sigma(y)\left|\partial_{y} u\left(y, F(y)-F\left(y_{0}\right)\right)\right|^{2}\right) d y
\end{gathered}
$$

and

$$
\mathcal{I}_{4}:=\frac{1}{2} \int_{y_{1}^{s,-}}^{y_{1}}\left(\rho(y)\left|\partial_{t} u\left(y, F\left(y_{1}\right)-F(y)\right)\right|^{2}+\sigma(y)\left|\partial_{y} u\left(y, F\left(y_{1}\right)-F(y)\right)\right|^{2}\right) d y .
$$

Due to the fact that $u^{0}=u^{1} \equiv 0$ in $\left(y_{0}, y_{1}\right)$, we have

$$
\mathcal{I}_{1}=0 \text {. }
$$


By the changes of variable $t \rightarrow y=y_{0}^{t,+}$ in $\mathcal{I}_{3}$ and $t \rightarrow y=y_{1}^{t,-}$ in $\mathcal{I}_{5}$ and due to the fact that A.44 implies that $F^{\prime}(y)=\sqrt{\rho(y) / \sigma(y)}$, we obtain

$$
-\mathcal{I}_{2}-\mathcal{I}_{3}=-\frac{1}{2} \int_{y_{0}}^{y_{0}^{s,+}}\left|\sqrt{\rho(y)} \partial_{t} u\left(y, F(y)-F\left(y_{0}\right)\right)+\sqrt{\sigma(y)} \partial_{y} u\left(y, F(y)-F\left(y_{0}\right)\right)\right|^{2} d y \leq 0
$$

and

$$
-\mathcal{I}_{4}+\mathcal{I}_{5}=-\frac{1}{2} \int_{y_{1}^{s,-}}^{y_{1}}\left|\sqrt{\rho(y)} \partial_{t} u\left(y, F\left(y_{1}\right)-F(y)\right)-\sqrt{\sigma(y)} \partial_{y} u\left(y, F\left(y_{1}\right)-F(y)\right)\right|^{2} d y \leq 0 .
$$

We conclude (A.45) by replacing A.50, A.51 and A.52 in the right hand side of A.49).

Acknowledgements. Both authors would like to thank Patrick Gérard (Université Paris-Sud) and Gilles Lebeau (Université de Nice Sophia-Antipolis) for useful suggestions and advices.

\section{REFERENCES}

[1] Bardos C., Lebeau G., Rauch J., Sharp sufficient conditions for the observation, control and stabilization of waves form the boundary, SIAM J. Control and Optimization, 30(1992), 1024-1065.

[2] Beckermann B., Serra-Capizzano S., On the asymptotic spectrum of finite element matrix sequences, SIAM J. Numer. Anal., 45(2)(2007), 746-769.

[3] Bühler O., A brief introduction to classical, statistical and quantum mechanics, Cournat Lecture Notes, Vol. 13, AMS, 2006.

[4] Burq N., Gérard P., Contrôle optimal des équations aux dérivées partielles, Course at École Polytéchnique, 2002.

[5] Castro C., Zuazua E., Concentration and lack of observability of waves in highly heterogeneous media, Arch. Rat. Mech. Anal., 164(1)(2002), 39-72.

[6] Cohen G., Higher-order numerical methods for transient wave equations, Springer, 2001.

[7] Cox S., Zuazua E., The rate at which energy decays in a damped string, Commun. in Partial Differential Equations, 19(1-2)(1994), $213-243$.

[8] Cox S., Zuazua E., The rate at which energy decays in a string damped at one end, Indiana Univ. Math. Journal, 44(2)(1995), $545-573$.

[9] Ervedoza S., On the mixed finite element method for the $1-d$ wave equation on non-uniform meshes, ESAIM:COCV, 2(2010), $298-326$.

[10] Ervedoza S., Spectral conditions for admissibility and observability of wave systems, Numerische Mathematik, 113(3)(2009), $377-415$

[11] Ervedoza S., Zuazua E., The wave equation: control and numerics, in Control and stabilization of PDEs, P. M. Cannarsa and J. M. Coron eds., Lecture Notes in Mathematics 2048, CIME Subseries, Springer Verlag, 2012, 245-340.

[12] Ervedoza S., Zuazua E., On the numerical approximation of exact controls for waves, Springer Briefs in Mathematics, XVII, 2013, ISBN 978-1-4614-5808-1.

[13] Evans L., Partial Differential Equations, Graduate Studies in Mathematics, Vol. 19, AMS, 2000.

[14] Gérard P., Oscillations and concentration effects in semilinear dispersive wave equations, J. Funct. Anal., 141(1996), 60-98.

[15] Gérard P., Markowich P.A., Mauser N.J., Poupaud F., Homogenization limits and Wigner transforms, Communications on Pure and Applied Mahematics, L(1997), 323-379.

[16] Jordan D.W., Smith P., Nonlinear ordinary differential equations. An introduction for scientists and engineers, Fourth edition, Oxford University Press, 2007.

[17] Keller J. B., Papanicolaou G., Ryzhik L., Transport equations for elastic and other waves in random media, Wave Motion, $24(1996), 327-370$.

[18] Kittel C., Introduction to Solid State Physics, Eight Edition, John Wiley \& Sons, 2005.

[19] Lax P. D., Richtmyer R. D., Survey of the stability of linear finite difference equations, Comm. Pure Appl. Math., 9(1956), $267-293$.

[20] Lions J.L., Contrôlabilité exacte, perturbations et stabilisation des systèmes distribués, vol. 1, Masson, Paris, 1988.

[21] Lions P.-L., Paul T., Sur les mesures de Wigner, Revista Matemática Iberoamericana, 9(3)(1993), 553-618.

[22] Macìa F., Propagación y control de vibraciones en medios discretos y continuos, PhD. Thesis, Universidad Complutense de Madrid, 2002.

[23] Macì F., Wigner measures in the discrete setting: high frequency analysis of sampling and reconstruction operators, SIAM J. Math. Anal., 36(2)(2004), 347-383.

[24] Macìa F., Zuazua E., On the lack of observability for wave equations: a Gaussian beam approach, Asymptotic Anal., 32(1)(2002), $1-26$.

[25] Marica A., Zuazua E., Boundary stabilization of numerical approximations of the $1-d$ variable coefficients wave equation: A numerical viscosity approach, submitted.

[26] Markowich P.A., Pietra P., Pohl C., Numerical approximation of quadratic observables of Schrödinger-type equations in the semi-classical limit, Numer. Math., 81(1999), 595-630.

[27] Markowich P.A., Poupaud F., The pseudo-differential approach to finite difference revisited, Calcolo, Springer-Verlag, 36(1999), $161-186$.

[28] Miller L., Escape function conditions for the observation, control, and stabilization of the wave equation, SIAM J. Cont. Optim., $41(5)(2003), 1554-1566$.

[29] Rebaza J., A first course in applied mathematics, Wiley, 2012.

[30] Serra-Capizzano S., Tablino Possio C., Analysis of preconditioning strategies for collocation linear systems, Linear Algebra and its Applications, 369(2003), 41-75. 
[31] Strogatz S.H., Nonlinear dynamics and chaos with applications to Physics, Biology, Chemistry and Engineering, Studies in nonlinearity, Perseus Books Publishing, 1994.

[32] Tilli P., Locally Toeplitz sequences: spectral properties and applications, Linear Algebra and its Applications, 278(1998), 91-120.

[33] Trefethen L.N., Group Velocity in Finite Difference Schemes, SIAM Review, 24(2)(1982), 113-136.

[34] Wigner E. P., On the quantum correction for thermodynamic equilibrium, Phys. Rev., 40(1932), 749-759.

[35] Zuazua E., Propagation, observation, control and numerical approximation of waves, SIAM Review, 47(2)(2005), 197-243.

Aurora Marica ${ }^{\S}, b$, Enrique ZuazuA ${ }^{\natural, \sharp}$

$\S$ Research Groups of Projects PN-II-ID-PCE-2012-4-0021 AND PN-II-ID-PCE-2011-3-0075, "Simion StoIlOW" Institute of Mathematics of the Romanian Academy, P.O. Box 1-764, 014700 Bucharest, Romania

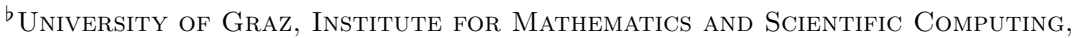

Heinrichstrasse 36, 8010, Graz, Austria,

ŁBCAM - Basque Center for Applied Mathematics,

Alameda Mazarredo 14, 48009, Bilbao, Basque Country, Spain,

AND

\#Ikerbasque - Basque Foundation for Science,

Alameda Urquijo 36-5, Plaza Bizkaia, 48011, Bilbao, Basque Country, Spain

E-mail address: aurora.marica@uni-graz.at, zuazua@bcamath.org

URL: https://sites.google.com/site/auroramihamarica/, www.bcamath.org/zuazua/ 